\title{
An AdS/QCD model from tachyon condensation: II
}

\author{
loannis latrakis, ${ }^{a}$ Elias Kiritsis ${ }^{a, b}$ and Ángel Paredes ${ }^{c}$ \\ ${ }^{a}$ Crete Center for Theoretical Physics, Department of Physics, University of Crete, \\ 71003 Heraklion, Greece \\ ${ }^{b}$ APC, Université Paris 7 , \\ Bâtiment Condorcet, F-75205, Paris Cedex 13 (UMR du CNRS 7164), France \\ ${ }^{c}$ Departament de Física Fonamental and ICCUB Institut de Ciències del Cosmos, \\ Universitat de Barcelona, Martí $i$ Franquès, 1, E-08028, Barcelona, Spain. \\ E-mail: iatrakis@physics.uoc.gr, kiritsis@physics.uoc.gr, \\ aparedes@ffn.ub.es
}

ABSTRACT: A simple holographic model is presented and analyzed that describes chiral symmetry breaking and the physics of the meson sector in QCD. This is a bottom-up model that incorporates string theory ingredients like tachyon condensation which is expected to be the main manifestation of chiral symmetry breaking in the holographic context. As a model for glue the Kuperstein-Sonnenschein background is used. The structure of the flavor vacuum is analyzed in the quenched approximation. Chiral symmetry breaking is shown at zero temperature. Above the deconfinement transition chiral symmetry is restored. A complete holographic renormalization is performed and the chiral condensate is calculated for different quark masses both at zero and non-zero temperatures. The $0^{++}, 0^{-+}, 1^{++}, 1^{--}$ meson trajectories are analyzed and their masses and decay constants are computed. The asymptotic trajectories are linear. The model has one phenomenological parameter beyond those of QCD that affects the $1^{++}, 0^{-+}$sectors. Fitting this parameter we obtain very good agreement with data. The model improves in several ways the popular hard-wall and soft wall bottom-up models.

KeYwords: AdS-CFT Correspondence, Tachyon Condensation, QCD, Spontaneous Symmetry Breaking

ARXIV EPRINT: 1010.1364 


\section{Contents}

1 Introduction 1

2 The gravitational background 3

3 The tachyon vacuum expectation value 4

3.1 The confined phase $\quad 6$

3.2 The deconfined phase 8

3.3 Holographic renormalization, the quark mass and the quark condensate 9

$\begin{array}{ll}3.4 & \text { The jump of the condensate at the phase transition } \\ \end{array}$

4 Meson excitations: the confined phase $\quad 12$

$\begin{array}{lll}4.1 \text { Vector mesons } & 13\end{array}$

$\begin{array}{lll}\text { 4.1.1 Schrödinger formalism and the mass spectrum } & 14\end{array}$

$\begin{array}{ll}\text { 4.1.2 Current-current correlator and normalization of the action } & 15\end{array}$

$\begin{array}{ll}\text { 4.1.3 Decay constants } & 17\end{array}$

4.1.4 Regge trajectories for vector mesons and linear confinement 18

$\begin{array}{ll}4.2 & \text { Axial-vector mesons } \\ & 19\end{array}$

$\begin{array}{lll}\text { 4.2.1 Schrödinger formalism and the mass spectrum } & 19\end{array}$

4.2.2 Current-current correlator and the pion decay constant 20

$\begin{array}{lll}4.3 & \text { Scalar mesons } & 21\end{array}$

4.4 Pseudoscalar mesons $\quad 22$

$\begin{array}{ll}\text { 4.4.1 The } m_{q}=0 \text { case } & 24\end{array}$

4.4.2 The $m_{q} \neq 0$ case 24

4.5 Mesonic excitations: a brief phenomenological analysis 25

$\begin{array}{lll}5 & \text { Meson melting in the deconfined phase } & 27\end{array}$

6 Discussion $\quad 29$

6.1 Summary and general comments on the model 29

6.2 Comments on effective actions for the open string tachyon 31

6.3 On the OPEs and the slope of the Regge trajectories 32

6.4 Outlook 33

A Book-keeping summary of the parameters of the model 34

B Analysis of singularities in the tachyon differential equation $\quad 35$

$\begin{array}{lll}\text { B.1 Confining background } & 35\end{array}$

$\begin{array}{lll}\text { B.2 Deconfined background } & 36\end{array}$

$\begin{array}{lll}\text { C Scheme dependence of the condensate and the constant } \alpha & 37\end{array}$

$\begin{array}{ll}\text { D Converting to Schrödinger form } & 39\end{array}$ 


\section{Introduction}

The AdS/CFT correspondence [1-3] has been one of the most fruitful arenas for research in fundamental physics in the last decade. Having the possibility of mapping stronglycoupled field theories to weakly coupled gravity has set the stage for a large amount of effort devoted to use this idea in order to obtain new results on the physics of the strong interactions (see [4], for an introduction). It is clear that a precise and controllable string dual of QCD is far from our present understanding. However, it is possible to build models that describe interesting strong coupling phenomena which share many similarities with real world physics. For the physics of pure glue, models descending from string theory, [5-8] have provided important clues towards the confinement of color. Phenomenological models for glue inspired and motivated from the AdS/CFT correspondence ranged from very simple like AdS/QCD [9] to more sophisticated versions, namely "Improved Holographic QCD" that capture rather accurately the dynamics of glue at both zero $[10,11]$ and finite temperature [12-15].

In this context, the holographic description of chiral symmetry breaking $(\chi \mathrm{SB})$ has been thoroughly studied. Early examples were studied in the Maldacena-Nunez [6, 7] and Klebanov-Strassler backgrounds [8]. Later, more QCD-like $\chi \mathrm{SB}$, in the sense that the operator condensing was bilinear of fundamental fields, was described in [16-18]. In the beginning chiral symmetry breaking involved abelian chiral symmetry, [6-8, 16-18]. A major breakthrough was the Sakai-Sugimoto model [19] where the broken symmetry is non-abelian $\mathrm{U}\left(N_{f}\right)_{L} \times \mathrm{U}\left(N_{f}\right)_{R} \rightarrow \mathrm{U}\left(N_{f}\right)_{V}$, as opposed to just a $\mathrm{U}(1)$. However, the model of [19] and generalizations of it have its own limitations. Just as an example, one can mention the absence of a tower of excited pions.

All the aforementioned models come from well controlled approximations to string theory and, accordingly, are top-down approaches. A different possibility is to use string theory just as inspiration and define ad hoc holographic models using some QCD features as inputs. This is the bottom-up approach. The obvious question is whether the output extracted from such models is larger than their input, and we believe that experience has shown that the answer is yes. The benchmark bottom-up models for chiral symmetry breaking and meson physics are the hard wall model [20, 21] based on the PolchinskiStrassler background [9] and the soft wall model [22].

In the present work, we analyze in detail several aspects of a bottom-up model presented first in [23]. It gives a explicit realization of the framework for chiral symmetry 
breaking first advocated in [24]: the quarks and antiquarks are introduced by a braneantibrane pair. A key point of the dynamics is the condensation of the lowest lying bifundamental scalar which comes from the strings connecting the brane to the antibrane. Around flat space this scalar has negative mass squared and this is called the tachyon. This corresponds precisely to a QCD-like chiral symmetry breaking. We need an effective action to describe this dynamics and we will resort to the tachyon-DBI action proposed by Sen in [25]. Once we have decided that we will use this action, we still have to choose an expression for the tachyon potential and a holographic geometry in order to use as curved background. For these choices, we will constrain ourselves to the (arguably) simplest possibilities. Another interesting property of considering brane-antibrane tachyonic actions is that there is a natural Wess-Zumino term, which correctly incorporates to the model features like parity and charge conjugation symmetries and anomalies; see the discussions in [24].

So far our ingredients are those of a top-down approach. However, it is not possible to stay in a limit in which the approximation to string theory is controlled if one wants to reproduce some QCD features in this context. For instance, we will need a background with curvature comparable to the string scale. We do not regard this point as a negative feature, but just as a sign that our approach should be considered of the bottom-up type. This has its own advantages, since for instance it seems impossible to get Regge trajectories for excited mesons $m_{n}^{2} \sim n$ from any top-down approach, because in those cases the meson mass scale is parametrically smaller than the QCD string tension scale, see for instance [26]. Therefore, the model discussed in this work should be regarded as a phenomenological model, partly inspired by top-down consideration and in particular by Sen's action [25]. These top-down inputs will generate some dynamics (compared to other bottom-up approaches) which will be crucial in the successful modeling of several QCD features, see the discussions in [24] and also in section 6.1.

We will constrain ourselves to the abelian case of a single quark flavor but, unlike [1618], this is not an essential limitation, since we can make the model non-abelian by piling up branes and antibranes, in the spirit of [19]. This elaboration, however, is left for future work.

In section 2, we will discuss the backgrounds (both for confined and deconfined phases) and the gravity action of which they are solutions. In section 3, we will study in detail the equation for the tachyon modulus $\tau$ and its bulk vacuum expectation value. In other words, we find the open string vacuum and show how it dynamically breaks the chiral symmetry. In section 4, we discuss in detail linearized open string excitations around the vacuum, namely the meson physics. A good review of this kind of analysis in different holographic frameworks is [27]. Apart from remarking several general qualitative properties, we end the section with a quantitative phenomenological analysis. Section 5 provides a brief analysis of the same kind of excitations, but in the deconfined phase. We conclude in section 6 with several discussions; we will convey the pros and cons of the present model and give some ideas for future directions. We have relegated various technical comments to eight appendices. In particular, in order to facilitate the reading of the text, we review the meaning of the different constants and parameters that will appear throughout the paper and for which physical reasons some of them are fixed in appendix A . 


\section{The gravitational background}

As acknowledged in the introduction, the model we will discuss does not come from any controlled approximation to string theory. Notwithstanding, we will follow general insights coming from string theory and effective actions developed in that framework, especially in the non-critical setting, [28-34]. In this sense, the meson physics (in the quenched approximation) is described by the dynamics of a D4-anti D4 system in a fixed closed string background, [31].

We take the following gravitational two-derivative action [28] for the background fields:

$$
S=\int d^{6} x \sqrt{g_{(6)}}\left[e^{-2 \phi}\left(\mathcal{R}+4(\partial \phi)^{2}+\frac{c}{\alpha^{\prime}}\right)-\frac{1}{2} \frac{1}{6 !} F_{(6)}^{2}\right],
$$

with a constant $c$. We consider the solution discussed in [29] whose metric is given by:

$$
d s_{6}^{2} \equiv-g_{t t} d t^{2}+g_{z z} d z^{2}+g_{x x} d x_{3}^{2}+g_{\eta \eta} d \eta^{2}=\frac{R^{2}}{z^{2}}\left[d x_{1,3}^{2}+f_{\Lambda}^{-1} d z^{2}+f_{\Lambda} d \eta^{2}\right]
$$

with:

$$
f_{\Lambda}=1-\frac{z^{5}}{z_{\Lambda}^{5}}
$$

This is the AdS soliton, a double Wick rotation of an $\mathrm{AdS}_{6}$ Schwartszchild black hole. The only active RR-form we consider is:

$$
F_{(6)}=\frac{Q_{c}}{\sqrt{\alpha^{\prime}}} \sqrt{-g_{(6)}} d^{6} x
$$

for some constant $Q_{c}$ which is proportional to the number of colors and that will not be important in the following. The dilaton is constant:

$$
e^{\phi}=\frac{1}{Q_{c}} \sqrt{\frac{2 c}{3}}
$$

The coordinate $\eta$ is compactified and regularity of the metric at $z=z_{\Lambda}$ requires the following periodicity condition:

$$
\eta \sim \eta+\delta \eta, \quad \delta \eta=\frac{4 \pi}{5} z_{\Lambda}=\frac{2 \pi}{M_{K K}} .
$$

The AdS radius is given by:

$$
R^{2}=\frac{30}{c} \alpha^{\prime}
$$

The application of this geometry for a phenomenological non-critical strings/gauge duality was first discussed in $[28,29]$. The solution is dual to $1+4$ dimensional gauge theory compactified in a circle with (susy-breaking) antiperiodic boundary conditions for the fermions. Thus, the low energy theory is $1+3$ dimensional confining gauge theory coupled to a set of massive Kaluza-Klein fields. 
One can consider the theory at non-zero temperature by compactifying to Euclidean time $t_{E}$. When both circles $t_{E}$ and $\eta$ are compactified, there is a second solution competing with $(2.2)$ :

$$
d s_{6}^{2}=\frac{R^{2}}{z^{2}}\left[-f_{T} d t^{2}+d x_{3}^{2}+f_{T}^{-1} d z^{2}+d \eta^{2}\right]
$$

while (2.4), (2.5), (2.7) still hold. We have introduced:

$$
f_{T}=1-\frac{z^{5}}{z_{T}^{5}}
$$

and $z_{T}$ is related to the period of the euclidean time and therefore to the temperature as:

$$
t_{E} \sim t_{E}+\delta t_{E}, \quad \delta t_{E}=\frac{4 \pi}{5} z_{T}=\frac{1}{T} .
$$

Since when we Euclideanize (2.2), (2.8) both solutions are related by the interchange $t_{E} \leftrightarrow$ $\eta, z_{T} \leftrightarrow z_{\Lambda}$, the symmetry makes obvious that there is a deconfining first order phase transition at

$$
T_{c}=\frac{M_{K K}}{2 \pi}=\frac{5}{4 \pi z_{\Lambda}}
$$

For $T<T_{c}$, the confining solution (2.2) is preferred and, conversely, (2.8) dominates for $T>T_{c}$. Of course, this discussion is just a straightforward generalization of [35].

\section{The tachyon vacuum expectation value}

Our main interest will be to study a "tachyon-DBI" action for a single brane-antibrane pair of the form advocated in [25]. In section 6.2 we will comment about the literature related to effective actions including open string tachyon fields and the possible impact of different choices of actions in a holographic model of this kind.

We take the brane-antibrane pair to be at a fixed value of $\eta$ and we will not consider oscillations of the transverse scalar, which has no QCD counterpart. ${ }^{1}$ The brane and antibrane are at zero distance and are therefore overlapping. We have therefore a 5D model for the quarks embedded in a $6 \mathrm{D}$ model for the glue. The Sen action reads:

$$
S=-\int d^{4} x d z V(|T|)\left(\sqrt{-\operatorname{det} \mathbf{A}_{L}}+\sqrt{-\operatorname{det} \mathbf{A}_{R}}\right)
$$

The quantities inside the square roots are defined as:

$$
\mathbf{A}_{(i) M N}=g_{M N}+\frac{2 \pi \alpha^{\prime}}{g_{V}^{2}} F_{M N}^{(i)}+\pi \alpha^{\prime} \lambda\left(\left(D_{M} T\right)^{*}\left(D_{N} T\right)+\left(D_{N} T\right)^{*}\left(D_{M} T\right)\right)
$$

where $(i)=L, R$ and the complex tachyon will be denoted $T=\tau e^{i \theta}$. Indices $M, N$ run over the 5 world-volume dimensions while we will use $\mu, \nu$ for the Minkowski directions (indices to be contracted using $\eta_{\mu \nu}$ ). With respect to [25], we have included two constants $g_{V}, \lambda$ in (3.2), which are related to the normalization of the fields to be discussed later.

\footnotetext{
${ }^{1} \mathrm{~A}$ different construction involving D4-anti D4 in this background was considered in [32-34]. The present scenario is more successful in describing different features of QCD.
} 
The covariant derivative of the tachyon field is defined as:

$$
D_{M} T=\left(\partial_{M}+i A_{M}^{L}-i A_{M}^{R}\right) T
$$

For the tachyon potential we take:

$$
V=\mathcal{K} e^{-\frac{1}{2} \mu^{2} \tau^{2}}
$$

where $\mathcal{K}$ is a constant ${ }^{2}$ which in principle should be related to the tension of the D4branes. The gaussian is a simple choice that has been discussed in different situations for instance in [36-38], but we warn the reader that it is not at all top-down derived for the present situation and thus should be considered as an ingredient of the bottom-up approach. We will comment further in section 6.2. For book-keeping, let us enumerate here the constants that have been introduced up to now: $R, \alpha^{\prime}, \lambda, g_{V}, \mathcal{K}, \mu, z_{\Lambda}$. In the following, we will impose, on physical grounds, some relations among these constants and in appendix A we will summarize these arguments.

We must first find the vacuum of the theory. We should set $\theta, A_{L}, A_{R}$ to zero because of Lorentz invariance, but $\tau$ must have non-trivial dynamics, at least in the confined phase, as will be argued below. We thus discuss here the function $\tau(z)$ that defines the vacuum. The corresponding reduced action reads:

$$
S=-2 \mathcal{K} \int d^{4} x d z e^{-\frac{1}{2} \mu^{2} \tau^{2}} g_{t t}^{\frac{1}{2}} g_{x x}^{\frac{3}{2}} \sqrt{g_{z z}+2 \pi \alpha^{\prime} \lambda\left(\partial_{z} \tau\right)^{2}}
$$

and the corresponding equation of motion:

$$
\tau^{\prime \prime}+\frac{\pi \alpha^{\prime} \lambda}{g_{z z}} \tau^{\prime 3}\left(\frac{g_{t t}^{\prime}}{g_{t t}}+3 \frac{g_{x x}^{\prime}}{g_{x x}}\right)+\frac{\tau^{\prime}}{2}\left(\frac{g_{t t}^{\prime}}{g_{t t}}+3 \frac{g_{x x}^{\prime}}{g_{x x}}-\frac{g_{z z}^{\prime}}{g_{z z}}\right)+\left(\frac{g_{z z}}{2 \pi \alpha^{\prime} \lambda}+\tau^{\prime 2}\right) \mu^{2} \tau=0
$$

We want to study this equation in both the confined and deconfined backgrounds of section 2. For this, we need to explicitly substitute the components of the metric of each background, as given in section 2. We will make these studies separately in the following subsections. Before that, since the UV of both solutions is identical (up to $\mathcal{O}\left(z^{5}\right)$ ), the analysis of the UV asymptotics of (3.6) is the same. We find that the near-boundary limit $z \rightarrow 0$ limit is given in terms of the two integration constants as:

$$
\tau=c_{1} z+\frac{\mu^{2}}{6} c_{1}^{3} z^{3} \log z+c_{3} z^{3}+\mathcal{O}\left(z^{5}\right)
$$

In order to find this expansion, we have imposed that:

$$
\frac{R^{2} \mu^{2}}{2 \pi \alpha^{\prime} \lambda}=3
$$

This enforces that the scalar bifundamental operator dual to the scalar field (which has mas $\left.m_{\tau}^{2}=-\mu^{2} /\left(2 \pi \alpha^{\prime} \lambda\right)\right)$ has UV dimension 3 matching the dimension of $\bar{q} q$ in QCD. This is in agreement with the usual AdS/CFT rule $\Delta(\Delta-4)=m_{\tau}^{2} R^{2}$. It is worth stressing

\footnotetext{
${ }^{2}$ We have included the constant dilaton in $\mathcal{K}$, in order to avoid unnecessary cluttering of formulae.
} 
that (3.8) should be understood as a bottom-up condition on the parameters determining the open string data $\mu, \alpha^{\prime}, \lambda$ and not on $R$, since in the quenched approximation one should not think of the flavor branes affecting the closed string background.

The asymptotic expansions for $\tau$ in the confined and deconfined backgrounds start differing at order $\mathcal{O}\left(z^{6}\right)$. On the other hand, the IR behaviour for both cases is very different, as will be discussed below.

\subsection{The confined phase}

Inserting the metric for the confining background (2.2) into (3.6), we obtain the following equation of motion for the order parameter:

$$
\tau^{\prime \prime}-\frac{4 \mu^{2} z f_{\Lambda}}{3} \tau^{\prime 3}+\left(-\frac{3}{z}+\frac{f_{\Lambda}^{\prime}}{2 f_{\Lambda}}\right) \tau^{\prime}+\left(\frac{3}{z^{2} f_{\Lambda}}+\mu^{2} \tau^{\prime 2}\right) \tau=0
$$

Before going on, notice that equation (3.9) depends on two constants $z_{\Lambda}$ and $\mu$. However, such dependence can be easily reabsorbed by redefining the field and radial coordinate as $z \rightarrow \tilde{z}=z / z_{\Lambda}, \tau \rightarrow \tilde{\tau}=\mu \tau$. The plots in this section will be performed by taking $z_{\Lambda}=1$, $\mu^{2}=\pi$, but it is automatic to find the solution for different values of the constants by rescaling as mentioned above.

According to the discussion of [24], since the background is confining, we must require the tachyon to blow up somewhere. Heuristically, one can think of the diverging tachyon as a brane-antibrane recombination; if the tachyon were finite until the bottom of the space one would have an open brane (and antibrane). In [24], it was argued that this would lead to bulk flavor anomalies that do not match those of QCD. ${ }^{3}$ The fact that confinement requires brane recombination (and therefore, chiral symmetry breaking) is a Coleman-Witten-like theorem [41] for the present set-up, and it is analogous to a similar discussion of [42] for the Sakai-Sugimoto model [19]. The difference is that the realization of chiral symmetry breaking in [42] is geometrical while here it is driven by the field $\tau$.

Equation (3.9) only allows the tachyon to diverge at exactly the end of space (the tip of the cigar) $z=z_{\Lambda}$, see appendix B for details.

In the IR, generically the two linearly independent solutions behave as a constant and $\sqrt{z-z_{\Lambda}}$ and they are regular at the tip. There is however a one parameter "boundary" family of solutions that (1) depends on a single parameter (2) diverges at the tip. This is the solution we should allow in the IR. If we call the single parameter $C$ then the acceptable IR solution is:

$$
\tau=\sum_{n=0}^{\infty}\left(z_{\Lambda}-z\right)^{\frac{3(2 n-1)}{20}} C_{n} g_{n}(z)
$$

where

$$
g_{n}(z)=1+\sum_{m=1}^{\infty} D_{n, m}\left(1-\frac{z}{z_{\Lambda}}\right)^{m}
$$

\footnotetext{
${ }^{3}$ Anomalies in the hard wall model have been discussed in $[39,40]$. In that case, appropriate IR boundary conditions have to be imposed on the gauge fields in order to get rid of the IR contribution to the gauge variation of the Chern-Simons term. In our case, that contribution is killed due to the divergent tachyon.
} 
For the first few constants we have

$$
\begin{aligned}
& C_{0}=C, \quad C_{1}=-\frac{13}{6 \mu^{2} C} \quad, \quad C_{2}=\frac{247}{72 \mu^{4} C^{3}} \quad, \quad C_{3}=-\frac{26975}{1296 \mu^{6} C^{5}} \\
& C_{4}=\frac{6381505}{31104 \mu^{8} C^{7}} \quad, \quad C_{5}=-\frac{276207997}{103680 \mu^{10} C^{9}} \quad, \quad C_{6}=\frac{1402840243831}{33592320 \mu^{12} C^{11}}
\end{aligned}
$$

and the first few functions

$$
\begin{aligned}
& g_{0}(z)=1-\frac{9}{20}\left(1-\frac{z}{z_{\Lambda}}\right)+\mathcal{O}\left(\left(1-\frac{z}{z_{\Lambda}}\right)^{2}\right) \\
& g_{1}(z)=1-\frac{1479}{3380}\left(\left(1-\frac{z}{z_{\Lambda}}\right)+\mathcal{O}\left(\left(1-\frac{z}{z_{\Lambda}}\right)^{2}\right)\right. \\
& g_{2}(z)=1-\frac{8481}{4940}\left(\left(1-\frac{z}{z_{\Lambda}}\right)+\mathcal{O}\left(\left(1-\frac{z}{z_{\Lambda}}\right)^{2}\right)\right. \\
& g_{3}(z)=1-\frac{396189}{82004}\left(\left(1-\frac{z}{z_{\Lambda}}\right)+\mathcal{O}\left(\left(1-\frac{z}{z_{\Lambda}}\right)^{2}\right)\right.
\end{aligned}
$$

As $C$ increases, the radius of convergence of this series increases.

The condition that the solution should end up in the one parameter family described above is our "regularity condition". It relates the two UV initial conditions, the source (mass) $c_{1}$ and the vev (chiral condensate) $c_{3}$. This is a dynamical determination of the condensate as a function of the mass by the condition $\tau\left(z=z_{\Lambda}\right)=\infty$. This relation will be found numerically.

In practice, one has to solve numerically the equation of motion (3.9) arranging the asymptotics to be (3.7) in the UV and (3.10) in the IR. One can implement a standard shooting routine whose inputs are $c_{1}$ and some UV and IR cutoffs, where the numerical solution is required to match the mentioned asymptotics. The value of $c_{3}$ leading to (3.10) is the limiting point between a behavior of diverging derivative of $\tau$ and a behaviour where $\tau$ remains finite everywhere, see figure 1 .

In fact, for fixed $c_{1}$ there are two values of $c_{3}$ for which $\tau$ diverges at $z_{\Lambda}$, since $\tau$ can diverge to $+\infty$, for a particular $c_{3}>0$; or to $-\infty$, for a particular $c_{3}<0$, (we are assuming by convention that $\left.c_{1}>0\right)$. However, the $c_{3}<0$ solution is unstable and should be discarded. This can be understood by comparing the free energy of both solutions or, alternatively, by realizing that there is a tachyonic mode in the pseudoscalar sector. In the massless quark case $c_{1}=0$, both solutions are related by $\tau \rightarrow-\tau$ and are physically equivalent. They are just related by a rotation in the direction of the Goldstone pion, which is exactly massless. This behaviour is completely analogous to the one described in [18]. For illustrative purposes, we plot in figure 1 the result of numerically integrating (3.9) and the behaviour of $\tau(z)$ for different values of $c_{3}$.

In figure 2, we plot the values of $c_{3}$ and $C$ obtained dynamically, as a function of $c_{1}$. 

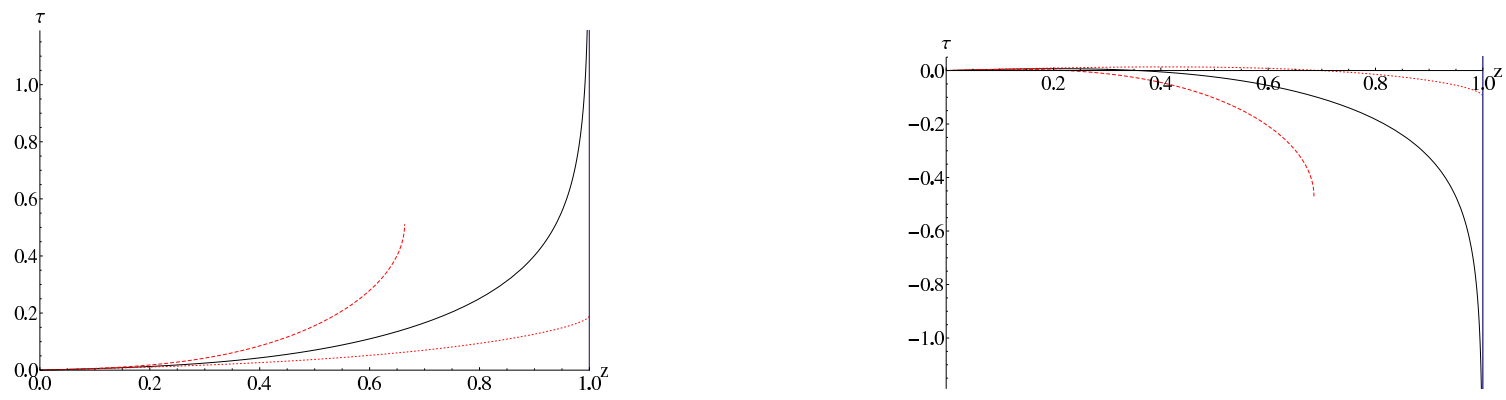

Figure 1. All the graphs are plotted using $z_{\Lambda}=1, \mu^{2}=\pi$ and $c_{1}=0.05$. The vertical line at $z=z_{\Lambda}=1$ represents the IR end of space (tip of the cigar). On the left, the solid black line represents a solution with $c_{3} \approx 0.3579$ for which $\tau$ diverges at $z_{\Lambda}$. The red dashed line has a too large $c_{3}$ - in particular, it corresponds to $c_{3}=1$ - such that there is a singularity at $z=z_{s}$ where $\partial_{z} \tau$ diverges while $\tau$ stays finite (a behaviour of the type $\tau=k_{1}-k_{2} \sqrt{z_{s}-z}$, that is unacceptable since the solution stops at $z=z_{s}$ where the energy density of the flavor branes diverges). The red dotted line corresponds to $c_{3}=0.1$; this kind of solution ought to be discarded because the tachyon stays finite everywhere. The plot in the right is done with the same conventions but with negative values of $c_{3}=-0.1,-0.3893,-1$. For $c_{3} \approx-0.3893$ there is a solution of the differential equation such that $\tau$ diverges to $-\infty$. As explained in the text, this solution is unstable. Thus, the physical solution for this particular value of $c_{1}$ is uniquely determined to be the solid line of the graph on the left.
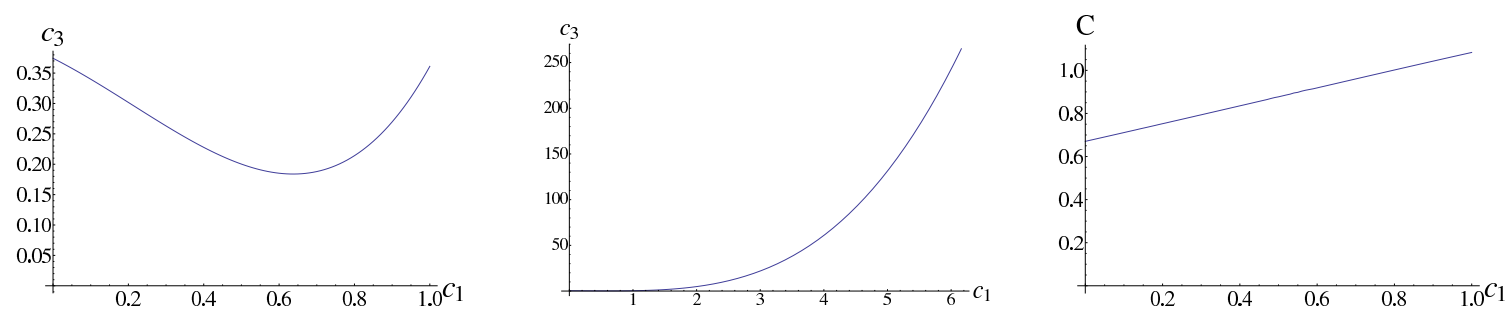

Figure 2. The values of $c_{3}$ and $C$ determined numerically as a function of $c_{1}$. In the first plot we portray $c_{3}$ in terms of $c_{1}$, for $c_{1} \leq 1$. In the second plot we show $c_{3}$ for a larger range of $c_{1}$. The third plot depicts the constant $C$ entering the IR expansion as a function of $c_{1}$. Again, we have used $z_{\Lambda}=1, \mu^{2}=\pi$ for the plots.

\subsection{The deconfined phase}

Inserting the metric (2.8) in (3.6), we obtain the following equation for $\tau$ in the deconfined phase:

$$
\tau^{\prime \prime}+\frac{\mu^{2} z^{2} f_{T}}{3} \tau^{\prime 3}\left(-\frac{4}{z}+\frac{f_{T}^{\prime}}{2 f_{T}}\right)+\left(-\frac{3}{z}+\frac{f_{T}^{\prime}}{f_{T}}\right) \tau^{\prime}+\left(\frac{3}{z^{2} f_{T}}+\mu^{2} \tau^{\prime 2}\right) \tau=0
$$

The IR behaviour of this equation is quite different from the one of (3.9). First of all $\tau$ is not allowed to diverge at any point. The difference with respect to the confining case is that since there is a horizon, one can allow the branes not to recombine as long as they end on the horizon. Then, they will not generate any anomaly.

Still, one has to discard solutions for which $\tau^{\prime}$ diverges at some $z<z_{T}$ (with $\tau$ remaining finite). Those solutions yield infinite energy density and are physically inconsistent, 

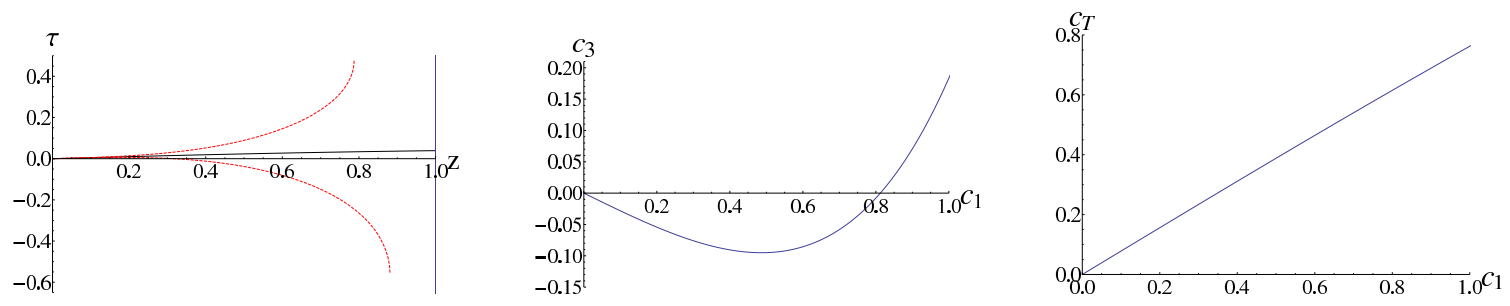

Figure 3. Plots corresponding to the deconfined phase. All the graphs are plotted using $z_{T}=1$, $\mu^{2}=\pi$. For the first plot we have taken $c_{1}=0.05$. The solid line displays the physical solution $c_{3}=-0.0143$ whereas the dashed lines $\left(c_{3}=-0.5\right.$ and $\left.c_{3}=0.5\right)$ are unphysical and end with a behaviour of the type $\tau=k_{1}-k_{2} \sqrt{z_{s}-z}$. The second and third plots give the values of $c_{3}$ and $c_{T}$ determined numerically by demanding the correct IR behaviour of the solution, as a function of $c_{1}$.

just as in the confining case. It turns out that this condition uniquely selects a value for $c_{3}$, for which $\tau$ reaches the horizon at $z=z_{T}$ taking there a finite value, say $\left.\tau\right|_{z=z_{T}}=c_{T}$, as shown in the first plot of figure 3 .

Thus, we have a one parameter family of physical solutions (which again fix $c_{3}$ in terms of $c_{1}$ as expected on physical grounds). In a similar fashion to the confined case, by redefining the field and radial coordinate as $z \rightarrow \tilde{z}=z / z_{T}, \tau \rightarrow \tilde{\tau}=\mu \tau$, the dependence of the equation on these two parameters can be reabsorbed. Near the IR, these solutions read, in terms of the parameter $\tau\left(z_{T}\right) \equiv c_{T}$ :

$$
\tau=c_{T}-\frac{3 c_{T}}{5 z_{T}}\left(z_{T}-z\right)-\frac{9 c_{T}}{200 z_{T}}\left(8+\mu^{2} c_{T}^{2}\right)\left(z_{T}-z\right)^{2}+\ldots
$$

Once $c_{1}$ is fixed, $c_{3}$ and $c_{T}$ are dynamically determined by this IR condition, and their values can be found numerically; using a standard shooting technique. Notice that for $c_{1}=0$, the solution is simply $\tau=0$ and chiral symmetry is unbroken. We display some plots with numerical results in figure 3 .

\subsection{Holographic renormalization, the quark mass and the quark condensate}

On general AdS/CFT grounds, we expect the integration constants $c_{1}, c_{3}$ of the UV expansion (3.7) to be related to the source and vacuum expectation value of the boundary operator associated to the bulk field $\tau$, which is the scalar quark bilinear $\bar{q} q$. Namely $c_{1}$ should be, essentially, the quark mass and $c_{3}$ the quark condensate. In this section, we will make this connection precise.

As has been pointed out many times - see for instance $[43,44]$ - in QCD the quark mass runs all the way to zero in the far UV, a fact that cannot be matched in a holographic model with AdS asymptotics (such that the $m_{q}$ we will define is the UV value, which does not run further). If we want to make a phenomenological analysis, the most natural option is to identify the $m_{q}$ of the model with the QCD quark mass measured at a scale around 1 or $2 \mathrm{GeV}$. It is conceivable that this feature can be ameliorated by using the tachyon action in a holographic setup which incorporates asymptotic freedom, as in "Improved holographic QCD" [10, 11]. 
The quark condensate is defined as:

$$
\langle\bar{q} q\rangle=-\frac{\delta S_{r e n}}{\delta m_{q}}
$$

where in order to find $S_{r e n}$ we have to follow the procedure of holographic renormalization, see for instance [48]. The first step is to regularize the action by placing a UV cut-off at $z=\epsilon$, namely $S_{\text {reg }}=\int_{\epsilon}^{z_{\Lambda}} \mathcal{L}$, where we have defined, from (3.5):

$$
\mathcal{L}=-2 \mathcal{K} e^{-\frac{1}{2} \mu^{2} \tau^{2}} g_{t t}^{\frac{1}{2}} g_{x x}^{\frac{3}{2}} \sqrt{g_{z z}+2 \pi \alpha^{\prime} \lambda\left(\partial_{z} \tau\right)^{2}}
$$

Since we are just concerned with the variation of $S_{\text {reg }}$ with respect to $m_{q}$, we compute the functional derivative with respect to $\tau$ :

$$
\delta S_{\text {reg }}=\int_{\epsilon}^{z_{\Lambda}}\left(\delta \tau \frac{\partial \mathcal{L}}{\partial \tau}+\delta \tau^{\prime} \frac{\partial \mathcal{L}}{\partial \tau^{\prime}}\right) d z=\int_{\epsilon}^{z_{\Lambda}} \frac{d}{d z}\left(\delta \tau \frac{\partial \mathcal{L}}{\partial \tau^{\prime}}\right)
$$

and therefore

$$
\frac{\delta S_{r e g}}{\delta \tau}=-\left.\frac{\partial \mathcal{L}}{\partial \tau^{\prime}}\right|_{z=\epsilon}
$$

We are interested in $\frac{\delta S_{\text {reg }}}{\delta c_{1}}=\frac{\delta \tau}{\delta c_{1}} \frac{\delta S_{\text {reg }}}{\delta \tau}$. In order to compute $\frac{\delta \tau}{\delta c_{1}}$, one should take into account that $c_{3}$ is a non-trivial function of $c_{1}$. We find by explicit computation, using the UV expansion (3.7):

$$
\frac{\delta S_{r e g}}{\delta c_{1}}=\mathcal{K} R^{5} \mu^{2}\left(\frac{2 c_{1}}{3 \epsilon^{2}}+\frac{2}{3} c_{1}^{3} \mu^{2} \log \epsilon+2 c_{3}-\frac{1}{3} c_{1}^{3} \mu^{2}+\frac{2}{3} c_{1} \partial_{c_{1}} c_{3}\right)
$$

where we have disregarded terms that vanish as $\epsilon \rightarrow 0$. We now have to write the appropriate covariant counterterms that should be added to $S_{r e g}$ in order to define the subtracted action $S_{\text {sub }}=S_{\text {reg }}+S_{c t}$ :

$$
S_{c t}=-\mathcal{K} R \int d^{4} x \sqrt{-\gamma}\left(-\frac{1}{2}+\frac{\mu^{2}}{3} \tau^{2}+\frac{\mu^{4}}{18} \tau^{4} \log \epsilon+\frac{\mu^{4}}{12} \alpha \tau^{4}\right)
$$

where $\gamma$ corresponds to the induced metric at $z=\epsilon$, namely $\sqrt{-\gamma}=R^{4} \epsilon^{-4}$. We have introduced a constant $\alpha$ which captures the scheme dependence of the condensate and reflects an analogous scheme dependence in field theory. It will be further discussed in appendix C. The renormalized action is just $S_{r e n}=\lim _{\epsilon \rightarrow 0} S_{s u b}$. It is now straightforward to find:

$$
\frac{\delta S_{\text {ren }}}{\delta c_{1}}=-\left(2 \pi \alpha^{\prime} \mathcal{K} R^{3} \lambda\right)\left(-4 c_{3}+c_{1}^{3} \mu^{2}(1+\alpha)\right)
$$

Notice that the term with $c_{1} \partial_{c_{1}} c_{3}$ in (3.23) drops out because there is one with the opposite sign in $\frac{\delta S_{c t}}{\delta c_{1}}$ that cancels it. We now want to evaluate the quark condensate (3.19). The quark mass is proportional to $c_{1}$, and we take it to be

$$
m_{q}=\beta c_{1}
$$

where $\beta$ is a constant.

The arbitrariness of this multiplicative constant related to the normalization of the fields has been stressed (in analogous situations) in [44, 49]. We finally obtain

$$
\langle\bar{q} q\rangle=\frac{1}{\beta}\left(2 \pi \alpha^{\prime} \mathcal{K} R^{3} \lambda\right)\left(-4 c_{3}+\left(\frac{m_{q}}{\beta}\right)^{3} \mu^{2}(1+\alpha)\right)
$$



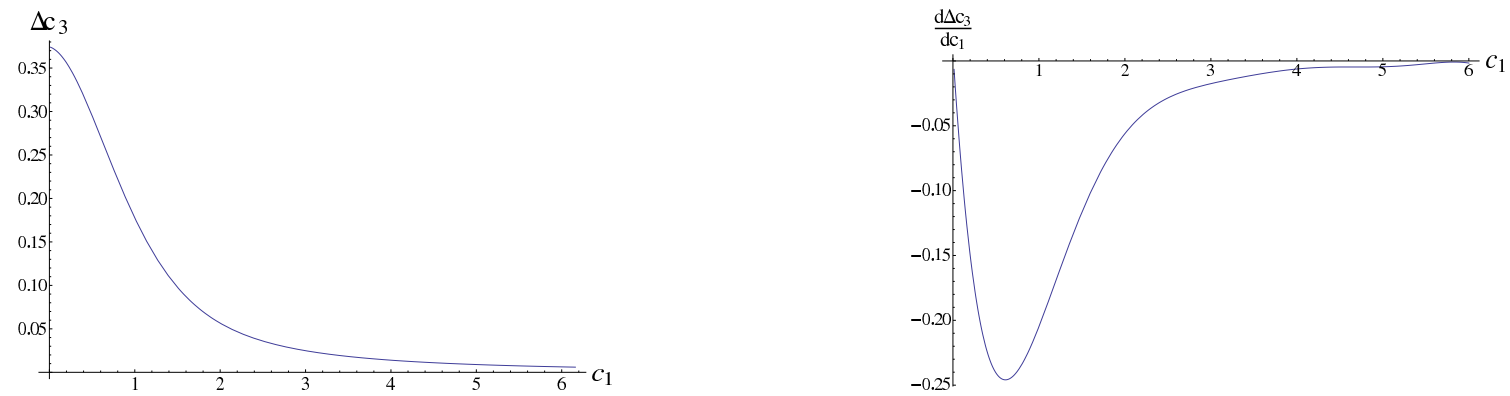

Figure 4. The finite jump of the quark condensate and its derivative with respect to $c_{1}$ when the confinement-deconfinement transition takes place. The values $z_{\Lambda}=z_{T}=1$ and $\mu^{2}=\pi$ have been used in the plot.

\subsection{The jump of the condensate at the phase transition}

The first term of the expression for the quark condensate (3.27) depends on the quantity $c_{3}$ that is determined dynamically via the numerical integration. The second term depends on the quark mass and a scheme dependent constant $\alpha$. We now compute an observable which is independent of this second term by finding the jump of the quark condensate when the theory is heated such that it undergoes the deconfinement phase transition. Concretely, we take a fixed mass (fixed $c_{1}$ ) and compare $c_{3}$ for a confined theory and deconfined theories, such that $z_{\Lambda}=z_{T}$, namely at the phase transition point. We have that $\Delta\langle\bar{q} q\rangle \equiv\langle\bar{q} q\rangle_{\mathrm{conf}}-\langle\bar{q} q\rangle_{\text {deconf }}=-4 \frac{1}{\beta}\left(2 \pi \alpha^{\prime} \mathcal{K} R^{3} \lambda\right) \Delta c_{3}$. In figure 4 we plot $\Delta c_{3}$, which in practice is nothing else that the difference between the first plot in figure 2 and the second plot of figure 3. It turns out to be a monotonously decreasing function, at least in the range of $c_{1}$ which we have been able to study numerically. We plot the result in figure 4 .

Let us now discuss how the quark condensate changes when tuning the temperature, while keeping fixed the quark mass and the QCD scale. We will plot the quantity:

$$
\langle\bar{q} q\rangle_{R}=\frac{m_{q}}{T_{c}^{4}}\left(\langle\bar{q} q\rangle_{T}-\langle\bar{q} q\rangle_{0}\right),
$$

where $\langle\bar{q} q\rangle_{T}$ is the condensate evaluated at temperature $T$. We have included the power of $T_{c}$ in the denominator in order to make the quotient dimensionless. Let us start by computing the explicit value of $\langle\bar{q} q\rangle_{0}$ from (3.27). We will consider small quark masses (compared to the QCD scale or $M_{K K}$ ) so we can neglect the last term of (3.27) and use the value $c_{3} \approx 0.37 z_{\Lambda}^{-3}$ computed for small $c_{1}$ in the first plot ${ }^{4}$ of figure 2 . Inserting the value of $z_{\Lambda}$ in terms of $T_{c}(2.11)$ and advancing the value of the normalization constant that will be found in (4.34), we have $\langle\bar{q} q\rangle_{0} \approx-0.3 N_{c} \beta T_{c}^{3}$. Turning on the temperature but staying below the phase transition, the functions of the metric do not depend on the temperature and therefore $\langle\bar{q} q\rangle_{R}=0$ for $T<T_{c}$. This is just a consequence of large- $N$ volume independence. In order to compute the result in the deconfined phase, we would like to use the values of $c_{3}$ as a function of $c_{1}$ plotted in figure 3. From the figure, one can

\footnotetext{
${ }^{4}$ We remind the reader that the plots were done by fixing $z_{\Lambda}=1$ and $z_{T}=1$ respectively. The values for generic $z_{\Lambda}, z_{T}$ are obtained just by rescaling $c_{1}=\left(c_{1} \mid z_{\Lambda}=1\right) z_{\Lambda}^{-1}$ and $c_{3}=\left(\left.c_{3}\right|_{z_{\Lambda}=1}\right) z_{\Lambda}^{-3}$, and similarly in the deconfined phase, substituting $z_{\Lambda}$ by $z_{T}$.
} 


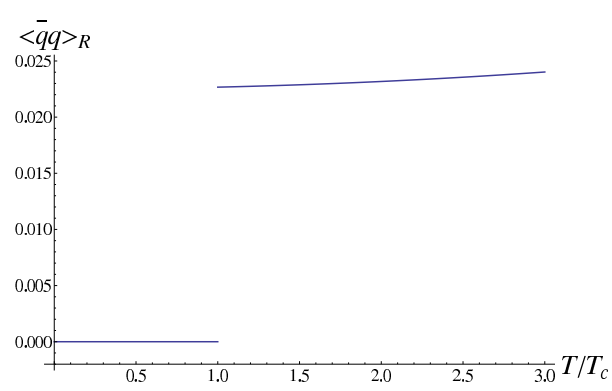

Figure 5. Behaviour of $\langle\bar{q} q\rangle_{R}$ as a function of the temperature. We have taken $N_{c}=3, \beta=1$, $m_{q} / T_{c}=1 / 40$ for the plot.

fit, for small $c_{1} z_{T}$ the value of $c_{3}$ to be $c_{3} z_{T}^{3} \approx-0.286 c_{1} z_{T}$. Using this expression, together with (3.26), (2.10), (4.34), we have $\langle\bar{q} q\rangle_{T} \approx 0.09 N_{c} m_{q} T^{2}$. Finally, we reach the result:

$$
\langle\bar{q} q\rangle_{R} \approx N_{c} \frac{m_{q}}{T_{c}^{4}}\left(0.3 \beta T_{c}^{3}+0.09 m_{q} T^{2}\right), \quad\left(T>T_{c}\right)
$$

We illustrate the behaviour of $\langle\bar{q} q\rangle_{R}$ in figure 5 . Notice that, since we are considering light quarks, the constant term is the largest until $T \gg T_{c}$.

This is in rough qualitative agreement with lattice results, see figure 4 of [50]. In our case, the jump at the phase transition is sharp due to the large $N$ limit.

\section{Meson excitations: the confined phase}

Up to now, we have discussed the vacuum (saddle point) of the model. We will now discuss in turn the different excitation modes, by expanding the action (3.1) up to quadratic order in all the fields. In this section, we will only refer to the confined phase and therefore $\langle\tau\rangle(z)$ is computed as in section 3.1.

We define the vector and axial vector fields as:

$$
V_{M}=\frac{A_{M}^{L}+A_{M}^{R}}{2}, \quad A_{M}=\frac{A_{M}^{L}-A_{M}^{R}}{2}
$$

The notation for the associated field strengths will be $V_{M N}, A_{M N}$. We use a gauge $A_{z}=$ $V_{z}=0$. We split the relevant fields as:

$$
\begin{aligned}
V_{\mu}\left(x^{\mu}, z\right) & =\psi^{V}(z) \mathcal{V}_{\mu}\left(x^{\mu}\right) \\
A_{\mu}\left(x^{\mu}, z\right) & =A_{\mu}^{\perp}\left(x^{\mu}, z\right)+A_{\mu}^{\|}\left(x^{\mu}, z\right)=\psi^{A}(z) \mathcal{A}_{\mu}\left(x^{\mu}\right)-\varphi(z) \partial_{\mu}\left(\mathcal{P}\left(x^{\mu}\right)\right) \\
\theta\left(x^{\mu}, z\right) & =2 \vartheta(z) \mathcal{P}\left(x^{\mu}\right) \\
\tau\left(x^{\mu}, z\right) & =\langle\tau\rangle(z)+s\left(x^{\mu}, z\right)=\langle\tau\rangle(z)+\psi^{S}(z) \mathcal{S}\left(x^{\mu}\right)
\end{aligned}
$$

where $\mathcal{V}_{\mu}$ and $\mathcal{A}_{\mu}$ are transverse vectors $\partial^{\mu} \mathcal{V}_{\mu}=\partial^{\mu} \mathcal{A}_{\mu}=0$. A few comments are in order: we have used the residual gauge freedom to make $V_{\mu}$ transverse. We have anticipated the behaviour of the equations of motion in order to write down the terms containing $\mathcal{P}(x)$, associated to the pseudoscalars. The symbol $\langle\tau\rangle(z)$ represents the tachyon vev in the bulk, as discussed in section 3 . 
The different bulk fields are dual to the field theory quark bilinears due to the boundary couplings: ${ }^{5} \int d^{4} x \mathcal{V}_{\mu} \mathcal{J}_{V}^{\mu}, \int d^{4} x \mathcal{A}_{\mu} \mathcal{J}_{A}^{\mu}, \int d^{4} x \mathcal{P} \mathcal{J}_{P}, \int d^{4} x \mathcal{S} \mathcal{J}_{S}$, where the $\mathcal{J}$ 's are the different bilinear quark currents: $\mathcal{J}_{V}^{\mu}=\bar{q} \gamma^{\mu} q, \mathcal{J}_{A}^{\mu}=\bar{q} \gamma^{\mu} \gamma^{5} q, \mathcal{J}_{P}=\bar{q} \gamma^{5} q, \mathcal{J}_{S}=\bar{q} q-\langle\bar{q} q\rangle$.

We also define the useful quantity:

$$
\tilde{g}_{z z}=g_{z z}+2 \pi \alpha^{\prime} \lambda\left(\partial_{z}\langle\tau\rangle\right)^{2}
$$

In the rest of this section, we will discuss the explicit prescriptions to compute the masses and decay constants for the different mesonic modes. In particular, the decay constants will require computing two-point correlators for which one has to holographically renormalize. We give here the complete set of counterterms which make the on-shell action finite.

$$
\begin{aligned}
& S_{c t}=-\mathcal{K} R \int d^{4} x \sqrt{-\gamma}\left(-\frac{1}{2}+\frac{\mu^{2}}{3} \tau^{2}+\frac{\mu^{4}}{18} \tau^{4} \log \epsilon+\frac{\mu^{4}}{12} \alpha \tau^{4}+\right. \\
& \left.+\frac{\left(2 \pi \alpha^{\prime}\right)^{2}}{g_{V}^{4}} \frac{1}{2} \gamma^{\mu \rho} \gamma^{\nu \delta}\left(V_{\mu \nu} V_{\rho \delta}+A_{\mu \nu} A_{\rho \delta}\right)\left(\log \epsilon+\frac{1}{2}\right)+\frac{R^{2} \mu^{2}}{3} \gamma^{\mu \nu}\left(D_{\mu} T\right)^{*}\left(D_{\nu} T\right)\left(\log \epsilon+\frac{1}{2}\right)\right)
\end{aligned}
$$

This expression completes (3.24) by including all the active fields we are considering. The terms of $1 / 2$ inside the brackets of the second line are finite contact terms that have been chosen for convenience.

We now discuss in turn each of the modes.

\subsection{Vector mesons}

The quadratic action corresponding to the vector mesons that comes from expanding (3.1) reads:

$$
S_{V}=-\frac{\left(2 \pi \alpha^{\prime}\right)^{2}}{g_{V}^{4}} \mathcal{K} \int d^{4} x d z e^{-\frac{1}{2} \mu^{2} \tau^{2}}\left[\frac{1}{2} \tilde{g}_{z z}^{\frac{1}{2}} V_{\mu \nu} V^{\mu \nu}+g_{x x} \tilde{g}_{z z^{-\frac{1}{2}}}^{-} \partial_{z} V_{\mu} \partial_{z} V^{\mu}\right]
$$

where we have constrained ourselves to the confining phase in which $g_{t t}=g_{x x}$. Here and in the following, it should be understood that the $\mu, \nu$ indices are contracted using the flat Minkowski metric, since we have explicitly written the factor of $g_{x x}=R^{2} / z^{2}$. The equation of motion can be easily derived:

$$
\frac{1}{e^{-\frac{1}{2} \mu^{2} \tau^{2}} \tilde{g}_{z z}^{\frac{1}{2}}} \partial_{z}\left(e^{-\frac{1}{2} \mu^{2} \tau^{2}} g_{x x} \tilde{g}_{z z^{-\frac{1}{2}}} \partial_{z} \psi^{V}(z)\right)-q^{2} \psi^{V}(z)=0
$$

where we have gone to Fourier space and defined the 4d-momentum such that for the eigenmodes it corresponds to the mass eigenvalues $q^{2}=-m_{V}^{2}$. The above equation explicitly depends on only two parameters $z_{\Lambda}$ and $\mu^{2}$. It is easy to check that $z_{\Lambda}$ just gives an overall scale to $m_{V}^{2}$ (and, in fact, to all dimensionful quantities that will appear later) and $\mu^{2}$ only enters through the combination $\tilde{\tau}^{2}=\mu^{2} \tau^{2}$. This was the same combination in the tachyon equation (see the comment below (3.9)), and in fact one can fix the value of $\mu^{2}$ without

\footnotetext{
${ }^{5}$ The various discrete symmetries and their realization are detailed in [24].
} 

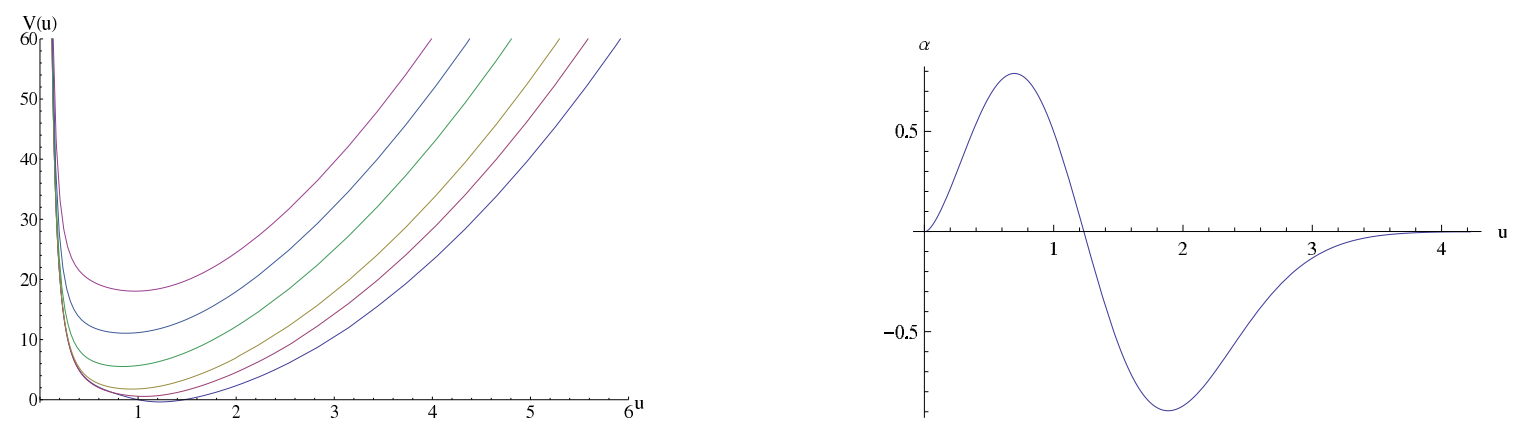

Figure 6. The Schrödinger potential associated to the vector excitation for different values of the quark mass. From bottom to top $c_{1}=0,0.5,1,2,3,4$. For illustrative purposes, on the right we plot the second normalizable "wavefunction" for $c_{1}=1$.

any loss of generality. From now on, we will set $\mu^{2}=\pi, z_{\Lambda}=1$ in all the plots, although we will keep the parameters explicit in the equations.

Finally, notice that (4.6) depends implicitly on $c_{1}$ (the quark mass) through the bulk vacuum expectation value of $\tau$. In short, the vector spectrum given by the model depends just on a multiplicative constant $z_{\Lambda}$ and the parameter $c_{1}$, namely the quark mass. All the other parameters that we have defined drop out from this computation.

\subsubsection{Schrödinger formalism and the mass spectrum}

In order to gain some insight in the problem, let us transform equation (4.6) to a Schrödinger problem, following appendix D. We immediately read $C(z)=M(z)=0$ and:

$$
A(z)=e^{-\frac{1}{2} \mu^{2} \tau^{2}} g_{x x} \tilde{g}_{z z}^{-\frac{1}{2}}, \quad B(z)=e^{-\frac{1}{2} \mu^{2} \tau^{2}} \tilde{g}_{z z}^{\frac{1}{2}},
$$

such that the Schrödinger radial variable is defined by:

$$
u=\int_{0}^{z} \sqrt{\frac{B(\tilde{z})}{A(\tilde{z})}} d \tilde{z}=\int_{0}^{z} \sqrt{\frac{\tilde{g}_{z z}(\tilde{z})}{g_{x x}(\tilde{z})}} d \tilde{z}
$$

Notice that $u \in[0, \infty)$. It is now a straightforward exercise to obtain the Schrödinger-like potential (D.7), for a given $c_{1}$. One has to compute numerically $\tau(z)$ as in section 3.1 , then evaluate (D.7) and finally implement the variable change (4.8). Some examples are plotted in figure 6.

We observe that the potentials move up as we increase $c_{1}$. This is of course expected on general grounds, since meson masses should grow with increasing quark masses, but this feature is missing from the hard wall or soft wall models. In [23], we made a phenomenological fit including the strange-strange meson masses in the analysis, finding good agreement with experimental data.

One can check that the leading contribution to $V(u)$ near $u=0$ is of the form $V(u)=$ $\frac{3}{4} u^{-2}+\ldots$ This just comes from the UV AdS asymptotics. Let us now find the leading IR contribution to $V(u)$. For large $u$ (namely near $z=z_{\Lambda}$ ), we have that $g_{x x} \approx R^{2} / z_{\Lambda}^{2}$ and $\tilde{g}_{z z} \approx 2 \pi \alpha^{\prime} \lambda\left(\partial_{z} \tau\right)^{2}$. Using the expressions in appendix D, a little algebra shows that 

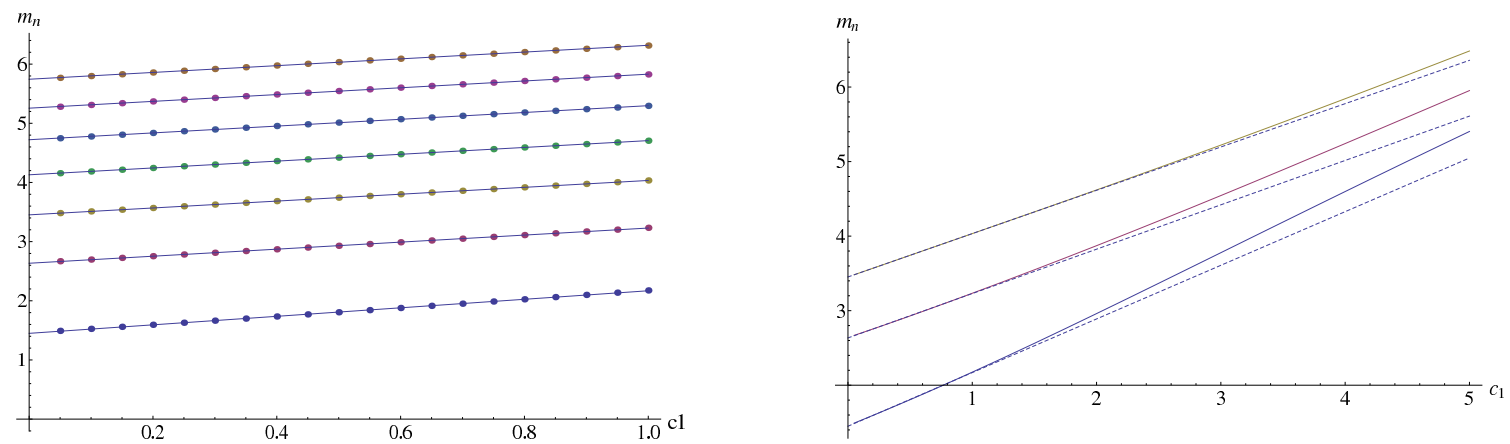

Figure 7. Vector meson masses as a function of $c_{1}$ (proportional to the quark mass). On the left, we plot the fitted straight lines together with several points computed numerically, for the seven lowest-lying vector modes, in the range $c_{1}<1$. On the right, we go to larger values of $c_{1}$ and, for clarity, only plot the three ligthest states. The dashed lines correspond to the linear fits valid for small $c_{1}$ whereas the solid line are the actual values found numerically.

for large $u$, we have $\frac{d u}{d z} \approx \frac{\mu}{\sqrt{3}} z_{\Lambda} \partial_{z} \tau$ and therefore $u \sim \frac{\mu}{\sqrt{3}} z_{\Lambda} \tau$. The function $\Xi$ behaves as $\Xi \approx\left(\frac{R}{z_{\Lambda}}\right)^{\frac{1}{2}} e^{-\frac{3 u^{2}}{4 z_{\Lambda}^{2}}}$ what finally leads to $V(u)=\frac{9}{4 z_{\Lambda}^{4}} u^{2}+\mathcal{O}(u)$. Therefore, $V(u)$ grows quadratically at large $u$, a fact that leads to standard Regge trajectories for large excitation number $n[22]$. Asymptotically, the slope of these trajectories is $\lim _{n \rightarrow \infty} \frac{d m_{n}^{2}}{d n}=\frac{6}{z_{\Lambda}^{2}}$, as can be found by evaluating (D.9).

By using standard numerical shooting techniques, we have computed the mass spectrum. In particular, we have computed the first seven states, changing the quark mass parameter in the range $0<c_{1}<5$. We plot some results in figure 7 . It turns out that for small $c_{1}$ the growth of meson mass on the quark mass is linear. This is just what one expects from a Taylor expansion if we consider the meson masses as function of the quark masses. This was a result already found in [23]. We have:

$$
\begin{aligned}
& z_{\Lambda} m_{V}^{(1)} \approx 1.45+0.718 c_{1}, \quad z_{\Lambda} m_{V}^{(2)} \approx 2.64+0.594 c_{1}, \quad z_{\Lambda} m_{V}^{(3)} \approx 3.45+0.581 c_{1}, \quad\left(c_{1} \leq 1\right) \\
& z_{\Lambda} m_{V}^{(4)} \approx 4.13+0.578 c_{1}, \quad z_{\Lambda} m_{V}^{(5)} \approx 4.72+0.577 c_{1}, \quad z_{\Lambda} m_{V}^{(6)} \approx 5.25+0.576 c_{1} .
\end{aligned}
$$

At around $c_{1} \geq 1$, the graphs start departing from the straight line, as can be seen on the second plot in figure 7 .

\subsubsection{Current-current correlator and normalization of the action}

We have discussed the vector spectrum, but we are also interested in the decay constants of each state. In order to compute them, we have to fix the multiplicative constant associated to the normalization of the action associated to the vector modes. We will follow the reasoning of $[20,21]$ and match the correlator $\Pi_{V}$ to the quark bubble perturbative computation at large Euclidean momentum. In fact, all the discussion of this subsection is completely parallel to $[20,21]$, since it only depends on the asymptotically AdS structure. We however repeat the argument in the present notation for the sake of clarity.

The current-current correlator is defined as:

$$
\int d^{4} x e^{i q x}\left\langle J_{\mu}(x) J_{\nu}(0)\right\rangle=\left(\eta_{\mu \nu} q^{2}-q^{\mu} q^{\nu}\right) \Pi_{V}\left(q^{2}\right) .
$$


As usual, we compute it holographically from the on-shell action. Integrating by parts in (4.5) and adding the counterterm from (4.4), we find:

$$
\begin{aligned}
S_{V}= & \left.\frac{\left(2 \pi \alpha^{\prime}\right)^{2} \mathcal{K}}{g_{V}^{4}} \int \frac{d^{4} q}{(2 \pi)^{4}} e^{-\frac{1}{2} \mu^{2} \tau^{2}} g_{x x} \tilde{g}_{z z}^{-\frac{1}{2}} V_{\mu}(q, z) \partial_{z} V^{\mu}(-q, z)\right|_{z=\epsilon}+ \\
& -\frac{\mathcal{K} R^{5}\left(2 \pi \alpha^{\prime 2}\right)}{g_{V}^{4}} \int \frac{d^{4} q}{(2 \pi)^{4}}\left(q^{2} V_{\mu}(q, \epsilon) V^{\mu}(-q, \epsilon)\left(\log \epsilon+\frac{1}{2}\right)\right)
\end{aligned}
$$

where $V_{\mu}(q, z)=\psi^{V}(q, z) V_{0}^{\mu}(q)$, and $\psi^{V}(q, z)$ is the solution to (4.6) subject to $V(q, \epsilon)=1$ and with normalizable behaviour in the IR. At small $z$, the solution for $\psi^{V}(q, z)$ can be expanded in terms of two integration constants as:

$$
\psi^{V}=b_{1}(q)+\left(b_{2}(q)+\frac{1}{2} b_{1}(q) q^{2} \log \frac{z}{z_{\Lambda}}\right) z^{2}+\ldots
$$

Substituting this expression into (4.11) and taking two derivatives with respect to $V_{0}^{\mu}(q)$, we find that:

$$
\Pi_{V}\left(q^{2}\right)=-4 \frac{\mathcal{K} R\left(2 \pi \alpha^{\prime}\right)^{2}}{g_{V}^{4}} \frac{b_{2}}{q^{2}}
$$

where we have set $b_{1}(q)=1$ consistent with the two-point function prescription and the non-trivial $q^{2}$-dependence comes through $b_{2}(q)$, which has to be found by integrating numerically and demanding the physical IR behaviour.

Before entering into numerical integration, we are interested in computing the limiting behaviour for $\Pi_{V}$ for large $q^{2}$. In order to do this, we consider again the equation written in Schrödinger form and notice that, for small $z$ :

$$
u \simeq z, \quad \alpha(u) \simeq u^{-\frac{1}{2}} \psi^{V}(u)
$$

The leading large $q$ behaviour is not affected by the details of the Schrödinger potential, so we may just approximate it by an expression that interpolates between its UV and IR behaviours, as discussed in subsection 4.1.1. Namely, we can just write:

$$
-\partial_{u}^{2} \alpha+\left(\frac{3}{4 u^{2}}+c^{2} u^{2}\right) \alpha+q^{2} \alpha=0,
$$

where we should take $c^{2}=\frac{9}{4 z_{\Lambda}^{4}}$. However, we will see that the value of $c^{2}$ does not matter for the normalization we want to make. (4.15) is nothing else than the soft wall model of [22]. The general solution of (4.15) is:

$$
\alpha(u)=k_{1} \frac{e^{\frac{-c u^{2}}{2}}}{\sqrt{u}} \mathrm{U}\left(\frac{q^{2}}{4 c}, 0, c u^{2}\right)+k_{2} \frac{e^{\frac{-c u^{2}}{2}}}{\sqrt{u}} L_{\frac{-q^{2}}{4 c}}^{-1}\left(c u^{2}\right)
$$

where $U$ stands for the confluent hypergeometric function and $L$ for a generalized Laguerre polynomial. IR normalisability requires $k_{2}=0$. We now substitute in (4.14) and fix $k_{1}$ by demanding that $\lim _{z \rightarrow 0} \psi^{V}(q, z)=\lim _{u \rightarrow 0} u^{\frac{1}{2}} \alpha(u)=1$.

$$
\psi^{V}(q, u)=\frac{q^{2}}{4 c} \Gamma\left(\frac{q^{2}}{4 c}\right) e^{\frac{-c u^{2}}{2}} \mathrm{U}\left(\frac{q^{2}}{4 c}, 0, c u^{2}\right)
$$




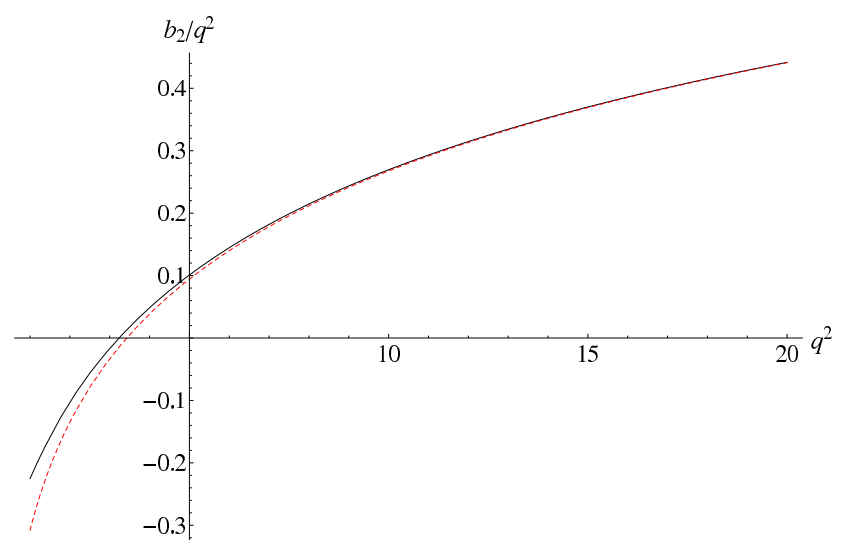

Figure 8. We plot the value of $b_{2} / q^{2}$, proportional to the vector current-current correlator. The plot is a comparison of the approximation found in the text from a simplified Schrödinger problem, eq. (4.18) (red dashed line) to the actual numerical result (solid line). The numerical plot was made by taking $c_{1}=0.1, \mu^{2}=\pi$.

We can now expand this expression for small $u \approx z$ and compare to (4.12), in order to read $b_{2}$ and, accordingly $\Pi_{V}$ from (4.13). The leading pieces at large $q^{2}$ for $b_{2}$ read:

$$
\lim _{q^{2} \rightarrow \infty} \frac{b_{2}}{q^{2}}=\frac{1}{4} \log \left(z_{\Lambda}^{2} q^{2}\right)-\frac{1}{4}(1+\log 4-2 \gamma)-\frac{c^{2}}{3 q^{4}}+\ldots,
$$

where $\gamma$ is Euler's constant. Therefore, the leading piece which we can compare to the quark bubble via (4.13) is:

$$
\Pi_{V}\left(q^{2}\right)=-\frac{\mathcal{K} R\left(2 \pi \alpha^{\prime}\right)^{2}}{g_{V}^{4}} \log \left(z_{\Lambda}^{2} q^{2}\right)
$$

By matching this expression to the perturbative result, we find: ${ }^{6}$

$$
\frac{\left(2 \pi \alpha^{\prime}\right)^{2} \mathcal{K} R}{g_{V}^{4}}=\frac{N_{c}}{12 \pi^{2}}
$$

One may wonder how good the results obtained from the simple Schrödinger problem we have discussed (4.15) are as an approximation to the full problem (4.6). In figure 8, we compare (4.18) to the value of $b_{2}\left(q^{2}\right) / q^{2}$ computed numerically.

\subsubsection{Decay constants}

We are now interested in determining the decay constants of our mesonic states. We start by writing the current-current correlator as a sum rule.

$$
\Pi_{V}\left(q^{2}\right)=\sum_{n} \frac{F_{n}^{2}}{\left(q^{2}+m_{n}^{2}-i \epsilon\right)}
$$

\footnotetext{
${ }^{6}$ Notice that we are dealing with abelian flavor symmetry. There is a factor of $\frac{1}{2}$ difference with respect to [20] since in that paper they deal with a non-abelian case and define $\operatorname{Tr}\left(t^{a} t^{b}\right)=\frac{1}{2} \delta^{a b}$. This also makes different the definition of the decay constants, for instance the $f_{\pi}$ defined in [20] is the $f_{\pi}$ we will use divided by $\sqrt{2}$.
} 


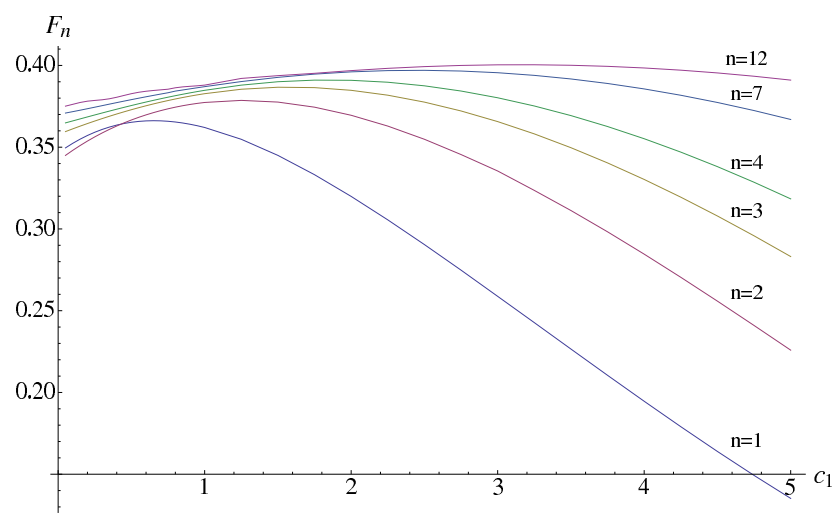

Figure 9. The decay constant, in units of $z_{\Lambda}^{-1}$ for the four lowest-lying, the seventh and the twelveth vector mode (from bottom to top), as a function of $c_{1}$. The numerical plot was made by taking $\mu^{2}=\pi$ and $N_{c}=3$.

The idea is to derive the form of the sum rule holographically. In appendix E we give a general description on how to write holographically a two-point correlator as an infinite sum. Then we use properties of the normalizable modes in order to determine the values of $F_{n}$. The argument follows $[20,21]$ so we directly quote the result in the present notation: ${ }^{7}$

$$
F_{n}^{2}=\frac{N_{c}}{6 \pi^{2}} \frac{R}{m_{n}^{2}}\left(\left.\frac{d^{2} \psi_{V}^{(n)}}{d z^{2}}\right|_{z=0}\right)^{2}
$$

where $\psi_{V}^{(n)}, n=1,2, \ldots, \infty$ are the solutions of (4.6) normalized as:

$$
\int B(z)\left(\psi_{V}^{(n)}\right)^{2} d z=1
$$

with $B(z)$ given in (4.7). Again, we can compute numerically the values of the decay constants given by the model. We have plotted them in figure 9 . One can see that the dependence on excitation number is rather mild for small quark masses and for a large number of modes.

\subsubsection{Regge trajectories for vector mesons and linear confinement}

Typical holographic models lead to a behaviour of the masses with the excitation number as $m_{n}^{2} \propto n^{2}$, for large $n$ [51]. However, experiment and semiclassical quantization of a hadronic string (assuming linear confinement) suggest that $m_{n}^{2} \propto n$ in QCD. Circumventing this problem was the motivation for developing the soft-wall model [22]. As pointed out above and also in [24], a model including an open string tachyon with action (3.1) and gaussian tachyon potential, naturally implements this behaviour. In figure 10, we plot the results of some numerical computations which display this feature. We remind the reader that, as we saw in section 4.1.1, for vector mesons $\lim _{n \rightarrow \infty} m_{n+1}^{2}-m_{n}^{2}=6 / z_{\Lambda}^{2}$. This seems to be born out by the figure.

\footnotetext{
${ }^{7}$ Notice our definition of $F_{n}$ is different from [20].
} 

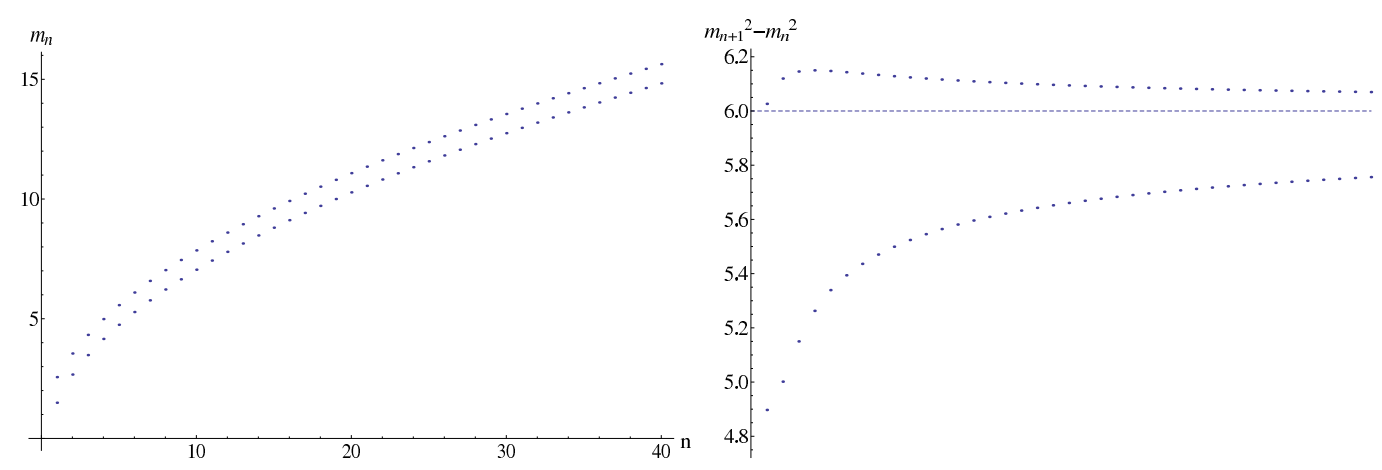

Figure 10. Results corresponding to the forty lightest vector states with $c_{1}=0.05$ and $c_{1}=1.5$. On the right, the horizontal line signals the asymptotic value 6 of the Regge trajectory, the lower line corresponds to $c_{1}=0.05$ and the upper line to $c_{1}=1.5$. Masses are given in units of $z_{\Lambda}^{-1}$.

\subsection{Axial-vector mesons}

The quadratic action corresponding to the axial vector mesons that comes from expanding (3.1) picks an extra term with respect to (4.5), coming from the covariant derivative of the tachyon:

$$
\begin{aligned}
S_{A}=-\frac{\left(2 \pi \alpha^{\prime}\right)^{2}}{g_{V}^{4}} & \mathcal{K} \int d^{4} x d z e^{-\frac{1}{2} \mu^{2} \tau^{2}} \\
\times & {\left[\frac{1}{2} \tilde{g}_{z z}^{\frac{1}{2}} A_{\mu \nu} A^{\mu \nu}+g_{x x} \tilde{g}_{z z^{2}}^{-\frac{1}{2}} \partial_{z} A_{\mu} \partial_{z} A^{\mu}+\frac{4 R^{2} g_{V}^{4}}{3\left(2 \pi \alpha^{\prime}\right)^{2}} \mu^{2} \tau^{2} g_{x x} \tilde{g}_{z z}^{\frac{1}{2}} A_{\mu} A^{\mu}\right] }
\end{aligned}
$$

The equation of motion can be derived to be:

$$
\frac{1}{e^{-\frac{1}{2} \mu^{2} \tau^{2}} \tilde{g}_{z z}^{\frac{1}{2}}} \partial_{z}\left(e^{-\frac{1}{2} \mu^{2} \tau^{2}} g_{x x} \tilde{g}_{z z^{-\frac{1}{2}}} \partial_{z} \psi^{A}(z)\right)-k \frac{\mu^{2} \tau^{2}}{z^{2}} \psi^{A}(z)-q^{2} \psi^{A}(z)=0
$$

where we have introduced a new constant $k$ as the combination:

$$
k=\frac{4 R^{4} g_{V}^{4}}{3\left(2 \pi \alpha^{\prime}\right)^{2}}
$$

We observe that $\tau$ only enters through the combination $\mu \tau$ so $\mu$ is immaterial since it can be rescaled away. On the other hand, the constant $k$, that did not enter the parity even sector does affect the physics. In fact, by comparing (4.6) to (4.25), one can see that the difference between the equation for the vectors and the one for the axials in controlled by $k$. Therefore, it is natural to guess that $k$ somehow enhances or suppresses the effects of chiral symmetry breaking on the P-odd spectra. In the following, we will see how the physics depends on this parameter. The model of our previous work [23] was more constrained since, in terms of the present notation, $k$ was fixed to $\frac{12}{\pi^{2}}$.

\subsubsection{Schrödinger formalism and the mass spectrum}

The functions for converting to a Schrödinger problem $A(z), B(z)$ are as before (4.7). On top of that, we have here a non-trivial $M(z)$ given by $M(z)=B(z) k \mu^{2} \tau^{2} / z^{2}$. It is easy 
to check that the leading piece in the UV of the Schrödinger potential is $3 / 4 u^{2}$ as for the vectors. However, the leading IR behaviour is modified due to the term proportional to $k$ to $V_{I R}(u)=\frac{9}{4 z^{4}}\left(1+\frac{4 k}{3}\right) u^{2}$. From this observation, one can immediately realize that the model gives different Regge slopes for vectors and axials and that the leading behaviour of $\Pi_{A}\left(q^{2}\right)$ at large Euclidean momentum coincides, consistently, with the vector one (4.19). We will later comment further on these issues.

The qualitative appearance of the Schrödinger potentials for the axial excitation is similar to the ones for the vectors. But the value of the potentials in the axial case is always higher due to the terms coming from $M(z)$. Thus, for equal excitation number and quark mass, the axial mode is always heavier than the vector mode (with the difference controlled by $k$ ). For small values of $c_{1}$, the dependence of the meson masses on the quark masses is linear

$$
\begin{aligned}
& z_{\Lambda} m_{A}^{(1)} \approx 2.05+1.46 c_{1}, \quad z_{\Lambda} m_{A}^{(2)} \approx 3.47+1.24 c_{1}, \quad z_{\Lambda} m_{A}^{(3)} \approx 4.54+1.17 c_{1}, \quad\left(c_{1} \leq 1\right) \\
& z_{\Lambda} m_{A}^{(4)} \approx 5.44+1.13 c_{1}, \quad z_{\Lambda} m_{A}^{(5)} \approx 6.23+1.11 c_{1}, \quad z_{\Lambda} m_{A}^{(6)} \approx 6.95+1.10 c_{1} .
\end{aligned}
$$

For this calculation, we used $k=\frac{18}{\pi^{2}}$ as it is found by the fit of the parameters in section (4.5), whereas in [23], we used $k=\frac{12}{\pi^{2}}$. For larger $c_{1}$, the plots of the meson mass dependence on the quark mass for the axial excitation look similar to the vector case, see the plot on the right of figure 7 .

\subsubsection{Current-current correlator and the pion decay constant}

By explicit computation it is easy to check that the UV expansion of the solution to (4.25) is given in terms of the two integration constants as:

$$
\psi^{A}=b_{1}+\left(b_{2}+\frac{1}{2} b_{1}\left(q^{2}+\pi k c_{1}^{2}\right) \log \frac{z}{z_{\Lambda}}\right) z^{2}+\ldots
$$

For the case of the axial vector, the corresponding sum rule generalising (4.21) reads:

$$
\Pi_{A}\left(q^{2}\right)=\frac{f_{\pi}^{2}}{q^{2}}+\sum_{n} \frac{F_{n}^{2}}{\left(q^{2}+m_{n}^{2}-i \epsilon\right)}
$$

where of course now the $n$ run over the axial resonances. The $F_{n}$ here are computed in essentially the same way as for the vector case, namely using (4.22) with a normalization condition (4.23).

Now, we are also be able to compute the value of $f_{\pi}$. We do this by directly computing the 2-point function at zero-momentum, namely:

$$
f_{\pi}^{2}=-\left.\frac{N_{c}}{6 \pi^{2}} b_{2}\right|_{q=0}
$$

where we have used the expansion (4.28), which up to that order, is also valid for the axial case. The value of $b_{2}$ to be inserted in (4.30) is found numerically by solving (4.25) with $q^{2}=0$, with initial condition $\left.\psi^{A}\right|_{z=\epsilon}=1$ and demanding IR normalizability.

From the figure, we observe that the decay constant grows with the quark mass for small quark masses and then starts decreasing. We can also observe that increasing the 

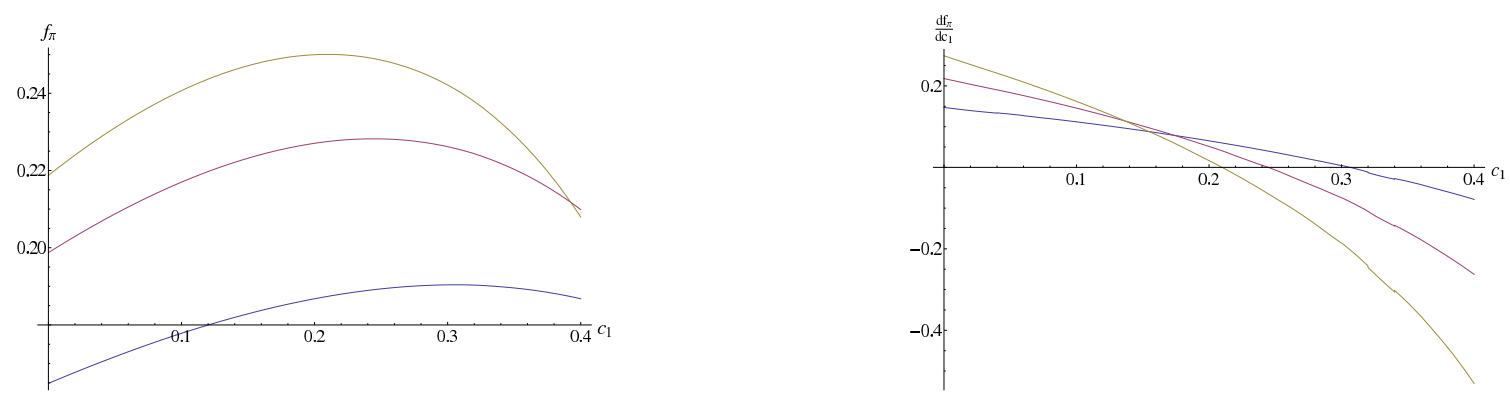

Figure 11. The pion decay constant and its derivative as a function of $c_{1}$ - the quark mass. The different lines correspond to different values of $k$. From bottom to top (on the right plot, from bottom to top in the vertical axis) $k=\frac{12}{\pi^{2}}, \frac{24}{\pi^{2}}, \frac{36}{\pi^{2}}$. The pion decay constant comes in units of $z_{\Lambda}^{-1}$.

parameter $k$, increases the value of the pion decay constant. This is in agreement with the intuitive notion given above that $k$ somehow controls the amount of chiral symmetry breaking.

\subsection{Scalar mesons}

We now deal with the scalar excitation. The quadratic action reads:

$$
\begin{array}{r}
S=-2 \pi \alpha^{\prime} \mathcal{K} \lambda \int d^{4} x d z e^{-\frac{1}{2} \mu^{2} \tau^{2}}\left[g_{x x}^{2} g_{z z} \tilde{g}_{z z}^{-\frac{3}{2}}\left(\partial_{z} s(x, z)\right)^{2}-2 \mu^{2} g_{x x}^{2} \tilde{g}_{z z}^{-\frac{1}{2}} \tau(z) \tau(z)^{\prime} s(x, z) \partial_{z} s(x, z)\right. \\
\left.+\frac{\mu^{2}}{2 \pi \alpha^{\prime} \lambda}\left(\mu^{2} \tau(z)^{2}-1\right) g_{x x}^{2} \tilde{g}_{z z}^{\frac{1}{2}} s(x, z)^{2}+g_{x x} g_{z z} \tilde{g}_{z z}^{-\frac{1}{2}}\left(\partial_{\mu} s(x, z)\right)^{2}\right]
\end{array}
$$

From (3.1), it can be seen that there is also a linear term in the bulk action, but can be easily shown to be a total derivative.

We can read the functions that are used to rewrite this problem is Schrödinger form, as defined in appendix D:

$$
\begin{array}{ll}
A(z)=e^{-\frac{1}{2} \mu^{2} \tau^{2}} g_{x x}^{2} \frac{g_{z z}}{\tilde{g}_{z z}^{3 / 2}}, & B(z)=e^{-\frac{1}{2} \mu^{2} \tau^{2}} g_{x x} \frac{g_{z z}}{\tilde{g}_{z z}^{1 / 2}}, \\
C(z)=-2 \mu^{2} e^{-\frac{1}{2} \mu^{2} \tau^{2}} \frac{g_{x x}^{2}}{\tilde{g}_{z z}^{1 / 2}} \tau(z) \partial_{z} \tau(z), & M(z)=\frac{\mu^{2}}{2 \pi \alpha^{\prime} \lambda} e^{-\frac{1}{2} \mu^{2} \tau^{2}}\left(\mu^{2} \tau^{2}-1\right) g_{x x}^{2} \tilde{g}_{z z}^{\frac{1}{2}} .
\end{array}
$$

Notice that $B(z) / A(z)$ takes the same value as for the vector and axial excitations, which means that the definition of the $u$-radial coordinate is the same as in those cases. The expression built from $B(z), C(z)$ and $M(z)$ which enters the Schrödinger potential takes a remarkably simple form:

$$
\frac{1}{B(z)}\left(M(z)-\frac{1}{2} \partial_{z} C(z)\right)=-\frac{3}{z^{2}}
$$

We will find the UV and IR limiting behaviour of the associated Schrödinger potential. At small $z \approx u$, we find $\Xi \approx R^{\frac{3}{2}} / u^{\frac{3}{2}}$ and one can immediately compute from (D.7) the UV leading term to be $V(u)=\frac{3}{4 u^{2}}$. Similarly, the term that dominates for large $u$ is quadratic $\frac{9 u^{2}}{4 z_{\Lambda}^{2}}$. Thus, we have found that the UV and IR asymptotics are the same as for 


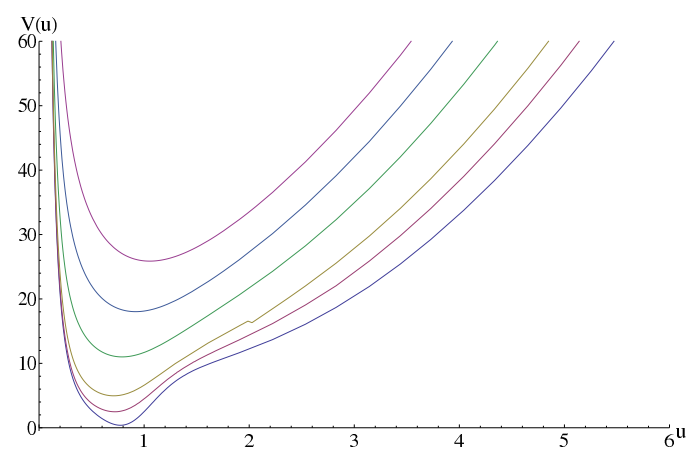

Figure 12. The Schrödinger potentials associated to the scalar excitation for $c_{1}=0,0.5,1,2,3,4$.

the vector case and, as a first approximation, we can use the soft wall equation (4.15). Thus, we can again match the asymptotic behaviour of the current-current correlator to the perturbative result. For that, we make an argument similar to [43] assume that the $q \bar{q}$ operator is dual to the tachyon rescaled by some constant $\beta$, such that the boundary coupling is, schematically, $\int \beta(\tau / z) q \bar{q}$. This is what we anticipated in the relation between $c_{1}$ and the quark mass (3.26). Then, matching the large $q^{2}$ result $\Pi_{S}\left(q^{2}\right)=\frac{N_{c}}{8 \pi^{2}} q^{2} \log q^{2}$ and reasoning as in the the vector case, we find:

$$
\frac{\left(2 \pi \alpha^{\prime}\right) \mathcal{K} R^{3} \lambda}{\beta^{2}}=\frac{N_{c}}{8 \pi^{2}}
$$

In figure 12, we depict the associated Schrödinger potential for different values of $c_{1}$. Comparing figure 12 to figure 6 , one can check that the potentials for the scalars are above those of the vectors. Thus, for equal excitation number, scalar mesons are typically heavier than vectors in the present model.

The relation of the lowest scalar meson masses to $c_{1}$ follows

$$
\begin{aligned}
& z_{\Lambda} m_{S}^{(1)} \approx 2.47+0.683 c_{1}, \quad z_{\Lambda} m_{S}^{(2)} \approx 3.73+0.488 c_{1}, \quad z_{\Lambda} m_{S}^{(3)} \approx 4.41+0.507 c_{1}, \quad\left(c_{1} \leq 1\right) \\
& z_{\Lambda} m_{S}^{(4)} \approx 4.99+0.519 c_{1}, \quad z_{\Lambda} m_{S}^{(5)} \approx 5.50+0.536 c_{1}, \quad z_{\Lambda} m_{S}^{(6)} \approx 5.98+0.543 c_{1} .
\end{aligned}
$$

We point out that the scalar meson masses do not depend on the parameter $k$.

\subsection{Pseudoscalar mesons}

We now focus on the pseudoscalar mesons. With respect to the previous modes, there is an extra complication because the physical modes are a combination of two bulk fields $\theta$ and $A_{\mu}^{\|}$. However, we will see that it is possible to find a combination of the fields for which one obtains a standard Sturm-Liouville problem.

The quadratic action reads:

$$
\begin{aligned}
S=-\left(2 \pi \alpha^{\prime}\right)^{2} \mathcal{K} \int d^{4} x d z e^{-\frac{1}{2} \mu^{2} \tau^{2}}\left[\frac{1}{g_{V}^{4}} g_{x x} \tilde{g}_{z z^{2}}^{-\frac{1}{2}}\left(\partial_{z} A_{\mu}^{\|}\right)^{2}\right. & \\
& \left.+\frac{\lambda}{2 \pi \alpha^{\prime}} \tau^{2} g_{x x}^{2} \tilde{g}_{z z^{-1}}^{-\frac{1}{2}}\left(\partial_{z} \theta\right)^{2}+\frac{\lambda}{2 \pi \alpha^{\prime}} \tau^{2} g_{x x} \tilde{g}_{z z}^{\frac{1}{2}}\left(\partial_{\mu} \theta+2 A_{\mu}^{\|}\right)^{2}\right]
\end{aligned}
$$


Defining $\varphi(z)$ and $\vartheta(z)$ as in (4.2) and Fourier transforming $\mathcal{P}\left(x^{\mu}\right)$, we can write the equations of motion for $A_{\mu}^{\|}$and $\theta$ as:

$$
\begin{aligned}
\frac{1}{e^{-\frac{1}{2} \mu^{2} \tau^{2}} \tilde{g}_{z z}^{\frac{1}{2}}} \partial_{z}\left(e^{-\frac{1}{2} \mu^{2} \tau^{2}} g_{x x} \tilde{g}_{z z}^{-\frac{1}{2}} \partial_{z} \varphi(z)\right)-k \frac{\mu^{2} \tau^{2}}{z^{2}}(\varphi(z)-\vartheta(z)) & =0 \\
k \frac{\mu^{2} \tau^{2}}{z^{2}} \partial_{z} \vartheta(z)+q^{2} \partial_{z} \varphi(z) & =0
\end{aligned}
$$

These two equations can be combined into one by solving (4.37) for $\vartheta$ and inserting this into (4.38).

$$
e^{-\frac{1}{2} \mu^{2} \tau^{2}} \tau^{2} g_{x x}^{2} \tilde{g}_{z z}^{-\frac{1}{2}} \partial_{z}\left[\frac{1}{e^{-\frac{1}{2} \mu^{2} \tau^{2}} \tau^{2} g_{x x} \tilde{g}_{z z}^{\frac{1}{2}}} \partial_{z} \psi^{P}(z)\right]-k \frac{\mu^{2} \tau^{2}}{z^{2}} \psi^{P}(z)-q^{2} \psi^{P}(z)=0
$$

where we have defined:

$$
\psi^{P}(z)=-e^{-\frac{1}{2} \mu^{2} \tau^{2}} g_{x x} \tilde{g}_{z z}^{-\frac{1}{2}} \partial_{z} \varphi(z)
$$

and we have used the definition of $k$ in (4.26). We can transform equation (4.39) to a Schrödinger form following appendix D. Comparing (4.39) to (D.2) (and inserting $C(z)=$ $0)$, we find:

$$
A(z)=e^{\frac{1}{2} \mu^{2} \tau^{2}} \tau^{-2} g_{x x}^{-1} \tilde{g}_{z z^{2}}^{-\frac{1}{2}}, \quad B(z)=e^{\frac{1}{2} \mu^{2} \tau^{2}} \tau^{-2} g_{x x}^{-2} \tilde{g}_{z z}^{\frac{1}{2}}, \quad \frac{M(z)}{B(z)}=k \frac{\mu^{2} \tau^{2}}{z^{2}}
$$

Notice that the value of $B / A$ coincides with those for the rest of modes and, therefore, the Schrödinger coordinate $u$ is the same for all the possible excitations. Let us compute the IR (large $u$ ) leading behaviour of such a Schrödinger potential. It turns out to be $V_{I R}(u) \approx \frac{9}{4 z_{\Lambda}^{4}}\left(1+\frac{4 k}{3}\right) u^{2}$, as for the axials. The coefficient of this quadratic term is what controls the slope of the Regge trajectories for highly excited mesons. Thus, the outcome of the present model in this respect is that vectors and scalars have the same Regge slope, whereas the slopes for axials and pseudoscalars coincide and are larger than the vector one.

An important observation is that the natural normalization condition is not (D.4) but, looking for the kinetic term of the pseudoscalar field in (4.36), we obtain:

$$
\left(2 \pi \alpha^{\prime}\right)^{2} \mathcal{K} \int_{0}^{z_{\Lambda}} d z e^{-\frac{1}{2} \mu^{2} \tau^{2}}\left[\frac{1}{g_{V}^{4}} g_{x x} \tilde{g}_{z z^{-}}^{-\frac{1}{2}}\left(\partial_{z} \varphi_{n}(z)\right)^{2}+\frac{4 \lambda}{2 \pi \alpha^{\prime}} \tau^{2} g_{x x} \tilde{g}_{z z}^{\frac{1}{2}}\left(\vartheta_{n}(z)-\varphi_{n}(z)\right)^{2}\right]=\frac{1}{2} .
$$

Rewriting this expression in terms of $\psi^{P}$, we find:

$$
\begin{aligned}
\frac{1}{2} & =\frac{\left(2 \pi \alpha^{\prime}\right)^{2} \mathcal{K}}{g_{V}^{4}} \int_{0}^{z_{\Lambda}} d z e^{\frac{1}{2} \mu^{2} \tau^{2}} g_{x x}^{-1}\left(\tilde{g}_{z z}^{\frac{1}{2}} \psi_{n}^{P}(z)^{2}+\frac{2 \pi \alpha^{\prime}}{4 \lambda g_{V}^{4} \tau^{2}} \tilde{g}_{z z^{2}}^{-\frac{1}{2}}\left(\partial_{z} \psi_{n}^{P}(z)\right)^{2}\right)= \\
& =\frac{\left(2 \pi \alpha^{\prime}\right)^{2} \mathcal{K}}{g_{V}^{4}} \int_{0}^{\infty} d u\left(g_{x x} \tau^{2} \alpha(u)^{2}+\frac{2 \pi \alpha^{\prime}}{4 \lambda g_{V}^{4}} e^{\frac{1}{2} \mu^{2} \tau^{2}} g_{x x}^{-\frac{3}{2}} \tau^{-2}\left[\partial_{u}\left(e^{-\frac{1}{4} \mu^{2} \tau} g_{x x}^{\frac{3}{4}} \tau \alpha(u)\right)\right]^{2}\right)
\end{aligned}
$$

where in the last line we have changed to the Schrödinger variables following the conventions of appendix D. There are some subtleties related to the UV behaviour of (4.43) which are worth explaining. Since the leading UV behaviour of our model is the same as in the hard 
wall $[20,21]$ or soft wall [22] models, the following arguments are analogous in all these cases. However, we are unaware of any reference where the discussion below is explicitly shown. It turns out that this UV behaviour is qualitatively different for massless $\left(c_{1}=m_{q}=0\right)$ or massive $\left(c_{1} \sim m_{q}>0\right)$ quarks. We will study both cases separately below.

\subsubsection{The $m_{q}=0$ case}

We will now study the qualitative properties of the physical spectrum for $m_{q}=0$. In this case, the $\tau \sim u^{3}$ near the UV and therefore $\Xi=(A B)^{\frac{1}{4}} \sim u^{-\frac{3}{2}}$, which implies that

$$
V_{U V}(u)=\frac{15}{4 u^{2}}+\ldots
$$

The first correction represented by the dots comes at order $u^{3}$. One can then find the UV expansion for $\alpha(u)$ that solves (D.6) in terms of the two integration constants which we denote $k_{1}, k_{2}$ as $\alpha(u)=k_{1} u^{-\frac{3}{2}}+\frac{1}{4} k_{1} m_{n}^{2} u^{\frac{1}{2}}-\frac{1}{16} k_{1} m_{n}^{4} u^{\frac{5}{2}} \log u+k_{2} u^{\frac{5}{2}}+\ldots$ We now want to insert this in the last line of (4.43) and check whether the integral converges near $u=0$. The first term is always convergent so we focus on the second term which behaves as $\int_{0} d u u^{-3}\left[\partial_{u}\left(u^{\frac{3}{2}} \alpha(u)\right)\right]^{2}$. Therefore, for $m_{n}=0$, this mode is UV-normalizable irrespective of the values of $k_{1}$ and $k_{2}$. Thus, one can always tune $k_{2} / k_{1}$ in order to find a solution that is well-behaved in the IR. This means that for $m_{q}=0$ there is always a normalizable solution with $m_{n}=0$, which corresponds to the expected massless Goldstone boson. On the other hand, if $m_{n} \neq 0$, one has to impose $k_{1}=0$ in order to have UV-normalizability. Then, as in a standard Sturm-Liouville problem, there will be a discrete set of massive modes, where $m_{n}$ is selected by matching the normalizable UV and IR behaviours.

In summary, for $m_{q}=0$ the UV structure of the Schrödinger potential and normalizability condition ensures the existence of a massless Goldstone boson together with a discrete tower of massive excitations, as expected.

\subsubsection{The $m_{q} \neq 0$ case}

Near the UV, we now have $\tau \sim u$ and therefore $\Xi=(A B)^{\frac{1}{4}} \sim u^{\frac{1}{2}}$, and

$$
V_{U V}(u)=-\frac{1}{4 u^{2}}+\ldots
$$

where the first correction in the dots is $\mathcal{O}\left(u^{0}\right)$. We can find again the UV solution in terms of two integration constants $\alpha(u)=k_{1} u^{\frac{1}{2}} \log u+k_{2} u^{\frac{1}{2}}+\mathcal{O}\left(u^{\frac{5}{2}}\right)$. Now, requiring that the last term of (4.43) is UV-finite requires setting $k_{1}=0$. Again, one has a Sturm-Liouville problem with a discrete spectrum.

Figure 13 depicts a few Schrödinger potentials for the pseudoscalar mode, for different values of $c_{1}$.

Let us now look at the lowest-lying excitation when $m_{q}$ is small. This should be a pseudo-Goldstone boson with its mass given by the Gell-Mann-Oakes-Renner relation. One indeed can find this following the argument of [20]: for zero quark mass, the $q^{2}=0$ solution of (4.37), (4.38) is given by $\vartheta(z)=-1, \varphi(z)=\psi_{q^{2}=0}^{A}(z)-1$, where we have defined $\psi_{q^{2}=0}^{A}(z)$ as the solution at zero momentum of (4.25) with boundary condition $\psi_{q^{2}=0}^{A}=1$. 

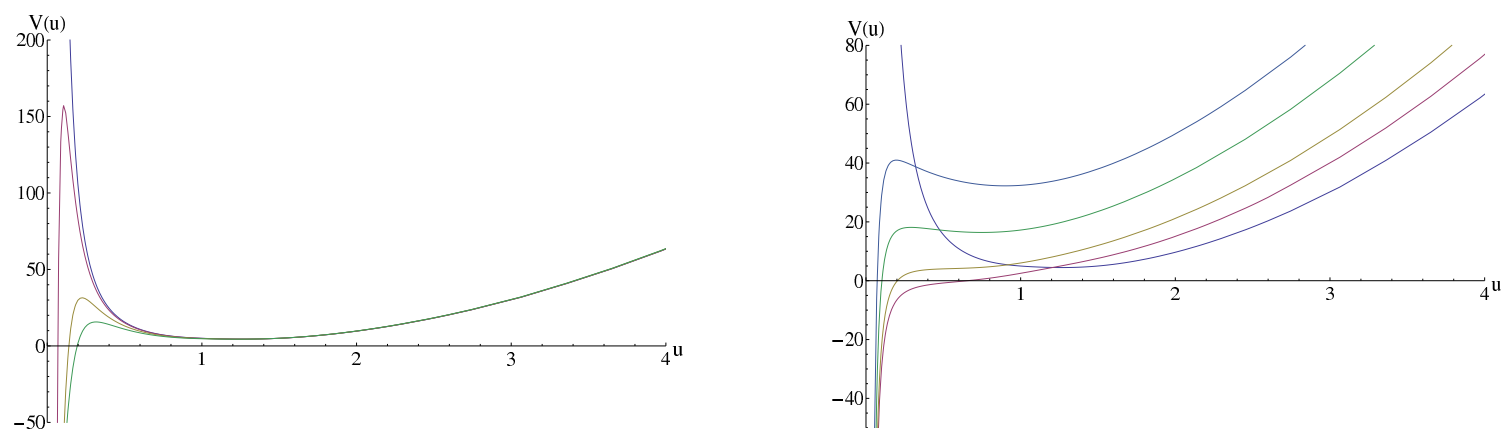

Figure 13. On the left, we plot the associated Schrödinger potential for small quark mass, concretely $c_{1}=0,0.001,0.005,0.01$. Even if the UV behaviour is completely different for $c_{1}=0$, we see that for small $c_{1}$, the potential looks very similar to the massless case except precisely around $u=0$. On the right, we plot the same but with larger values of $c_{1}$, in particular $c_{1}=0,0.5,1,2,3$. All plots have been done taking $k=\frac{12}{\pi^{2}}$. As for the axial case, increasing $k$ amounts to pushing up the IR part of the potential.

Consequently, regarding (4.30), $\left.z^{-1} \partial_{z} \psi_{q^{2}=0}^{A}\right|_{z=0}=-\frac{6 \pi^{2}}{N_{c}} f_{\pi}^{2}$. We now find perturbatively the small $m_{\pi}^{2}$ solution just by integrating (4.38) and we obtain:

$$
-1=m_{\pi}^{2} \int \frac{z^{2}}{\mu^{2} k \tau^{2}} \partial_{z} \psi_{q^{2}=0}^{A} d z
$$

Using that this integral is dominated by the small $z$ region and taking into account $\int \frac{z^{3}}{\tau^{2}} \approx$ $\int \frac{z^{3}}{\left(c_{1} z+c_{3} z^{3}\right)^{2}} \approx \frac{1}{2 c_{1} c_{3}}$, we can substitute the relations between $c_{1}, c_{3}$ and $m_{q},\langle\bar{q} q\rangle(3.26)$, (3.27) together with (3.8), (4.20) and (4.26) to find the GOR relation [52]:

$$
-4 m_{q}\langle q \bar{q}\rangle=m_{\pi}^{2} f_{\pi}^{2}
$$

We have obtained this expression by making a series of approximations. However, we can crosscheck it with the values for the mass obtained by the standard numerical computation, see figure 14. (4.47) is very accurate for small masses. When going to larger masses (up to $c_{1} \approx 1$ ), we can fit the mass of the lowest lying pseudoscalar to $\sqrt{a m_{q}+b m_{q}^{2}}$. We include here the masses of the first six pseudoscalar modes in terms of $c_{1}$

$$
\begin{aligned}
& z_{\Lambda} m_{P}^{(1)} \approx \sqrt{3.53 c_{1}^{2}+6.33 c_{1}}, \quad z_{\Lambda} m_{P}^{(2)} \approx 2.91+1.40 c_{1}, \quad z_{\Lambda} m_{P}^{(3)} \approx 4.07+1.27 c_{1}, \quad\left(c_{1} \leq 1\right) \\
& z_{\Lambda} m_{P}^{(4)} \approx 5.04+1.21 c_{1}, \quad z_{\Lambda} m_{P}^{(5)} \approx 5.87+1.17 c_{1}, \quad z_{\Lambda} m_{P}^{(6)} \approx 6.62+1.15 c_{1} .
\end{aligned}
$$

where we have also set $k=\frac{18}{\pi^{2}}$ for this calculation.

\subsection{Mesonic excitations: a brief phenomenological analysis}

We now make a phenomenological analysis of our model by comparing our results for the spectrum and the decay constants of light unflavored mesons to their experimental values. An extensive study of meson spectrum appeared in [23], without including the decay constants. We will fit the three parameters of the model, $z_{\Lambda}, c_{1}$ and $k$, using mesons with isospin 1 and $J^{P C}=1^{--}, 1^{++}, 0^{-+}, 0^{++}$. Since $z_{\Lambda} \sim \Lambda_{Q C D}^{-1}$ and $c_{1} \sim m_{q}$, it turns out 

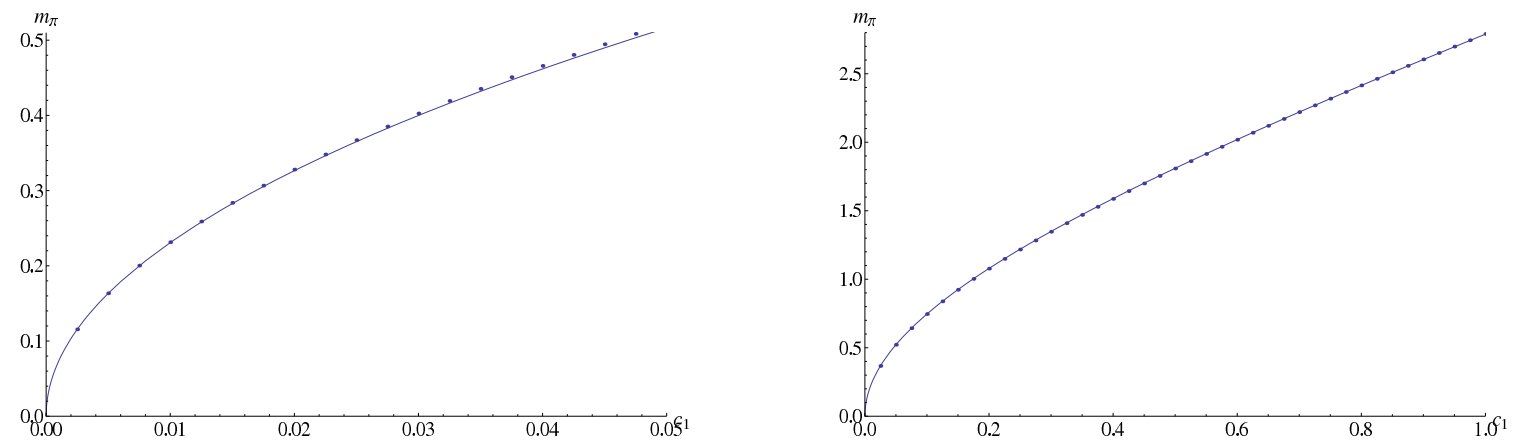

Figure 14. We plot the mass of the lowest lying pseudoscalar as a function of $c_{1}$ (namely, the quark mass). On the left, we crosscheck the GOR relation (where for $\langle q \bar{q}\rangle, f_{\pi}$ we have introduced the result found numerically in the chiral limit) to some points computed numerically. The approximation is very good for small $c_{1}$, and deviations start seeing visible around $c_{1}=0.03$. On the right, we have fitted $b$ in the expression $m_{\pi}=\sqrt{\frac{-4 m_{q}\langle q \bar{q}\rangle}{f_{\pi}^{2}}+b m_{q}^{2}}$ and checked that the fit is rather good up to $c_{1}=1$. the parameter $k$ has been taken to be $\frac{12}{\pi^{2}}$ in these plots.

\begin{tabular}{|c|c|c|c|c|}
\hline$J^{C P}$ & Meson & Measured $(\mathrm{MeV})$ & Model $(\mathrm{MeV})$ & $100|\delta O| / O$ \\
\hline \multirow{2}{*}{$1^{--}$} & $\rho(770)$ & 775 & 800 & $3.2 \%$ \\
\cline { 2 - 5 } & $\rho(1450)$ & 1465 & 1449 & $1.1 \%$ \\
\hline $1^{++}$ & $a_{1}(1260)$ & 1230 & 1135 & $7.8 \%$ \\
\hline $0^{-+}$ & $\pi_{0}$ & 135.0 & 134.2 & $0.5 \%$ \\
\cline { 2 - 5 } & $\pi(1300)$ & 1300 & 1603 & $23.2 \%$ \\
\hline $0^{++}$ & $a_{0}(1450)$ & 1474 & 1360 & $7.7 \%$ \\
\hline
\end{tabular}

Table 1. The results of the model and the experimental values for light unflavored meson masses.

that there is a single phenomenological parameter $k$, apart from those inherent of QCD physics.

The experimental values of the meson masses which are used are quoted by [53].

We fit the three parameters of our model to the masses of the light mesons which appear in table 1 and the decay constants appearing in 2. To make the fit we minimize the rms error

$$
\epsilon_{r m s}=\left(\frac{1}{n} \sum_{i}\left(\frac{\delta O_{i}}{O_{i}}\right)^{2}\right)^{\frac{1}{2}}
$$

where $n$ is the number of the observables minus the number of the fitted parameters, $n=9-3$. The values of the parameters minimizing $\epsilon_{r m s}$ read

$$
z_{\Lambda}^{-1}=549 \mathrm{MeV} \quad, \quad c_{1 l} z_{\Lambda}=0.0094 \quad, \quad k=\frac{18}{\pi^{2}}
$$

The rms error then is $\epsilon_{r m s}=14.5 \%$ and the comparison between the experimental and model values appears in table 1 , for the masses and in table 2 , for the decay constants. 


\begin{tabular}{|c|c|c|c|c|}
\hline$J^{C P}$ & Meson & Measured $(\mathrm{MeV})$ & Model $(\mathrm{MeV})$ & $100|\delta O| / O$ \\
\hline $1^{--}$ & $\rho(770)$ & 216 & 190 & $12 \%$ \\
\hline $1^{++}$ & $a_{1}(1260)$ & 216 & 228.5 & $5.8 \%$ \\
\hline $0^{-+}$ & $\pi_{0}$ & 127 & 101.3 & $20.2 \%$ \\
\hline
\end{tabular}

Table 2. A comparison of the results to the experimental values for the decay constants of light unflavored mesons.

\section{Meson melting in the deconfined phase}

We briefly discuss in this section the fate of the mesonic modes when the gauge theory undergoes a deconfining phase transition [54], namely when we use the background of equation (2.8). The first observation is that, as we saw in section 3.2, the tachyon cannot diverge at any point in this case and, therefore, the brane reaches the horizon, and we only have "black hole embeddings", in analogy with the terminology introduced in $[55,56]$. This means that there is no discrete spectrum above the deconfining phase transition. When we are considering small quark masses, this is perfectly realistic.

However, in the real world, charmonium and bottomonium do survive the QCD phase transition. We want to study this problem in the present model, and therefore we will compute the spectral functions at different values of $m_{q} / T$. In particular, we will focus in the vector excitation.

We start by discussing the associated Schrödinger potential for the vector excitation in the deconfined background, at zero momentum. The expressions in (4.7) are modified to:

$$
A(z)=e^{-\frac{1}{2} \mu^{2} \tau^{2}} g_{x x}^{\frac{1}{2}} g_{t t}^{\frac{1}{2}} \tilde{g}_{z z^{-\frac{1}{2}}}, \quad B(z)=e^{-\frac{1}{2} \mu^{2} \tau^{2}} g_{x x}^{\frac{1}{2}} g_{t t}^{-\frac{1}{2}} \tilde{g}_{z z}^{\frac{1}{2}}
$$

where one should remember that now $g_{\mu \nu}$ refers to the metric (2.8). Notice that $\sqrt{B / A}$ diverges at $z=z_{T}$ as a single pole, such that $\int \sqrt{B / A} d z$ diverges and the horizon $z=z_{T}$ corresponds to $u=\infty$ in the Schrödinger coordinate. $V(u)$ is exponentially decreasing for large $u$. For completeness, we write in appendix $\mathrm{G}$ the functions determining the potential for the rest of modes. In figure 15, we show several examples of potentials computed numerically, for different values of $c_{1} \sim m_{q} z_{T} \sim m_{q} / T$. In the second and third plots, we also compare it to the potentials in the confined phase for the same value of $c_{1}$. Namely, we show how the potentials for the vector excitations are modified at the phase transition. They share the same UV behaviour, but are drastically modified in the IR due to the different behaviour of the tachyon and the metric.

In [59], it was shown that a step-like potential gives quasi-particle behaviour. Moreover, if the position of the step coincides with that of a barrier in the confined phase, the quasiparticle mass is related to the mass of the meson before the phase transition. The third plot seems to point along that line. However, in the present model the potentials for the deconfined phase are never step-like enough, nor present bumps, and thus do not create sharp peaks in the spectral function, as we will see below.

Once we have the potentials, it is straightforward to compute the spectral function from the retarded correlator which is computed following the prescription of [60]. In practice, 

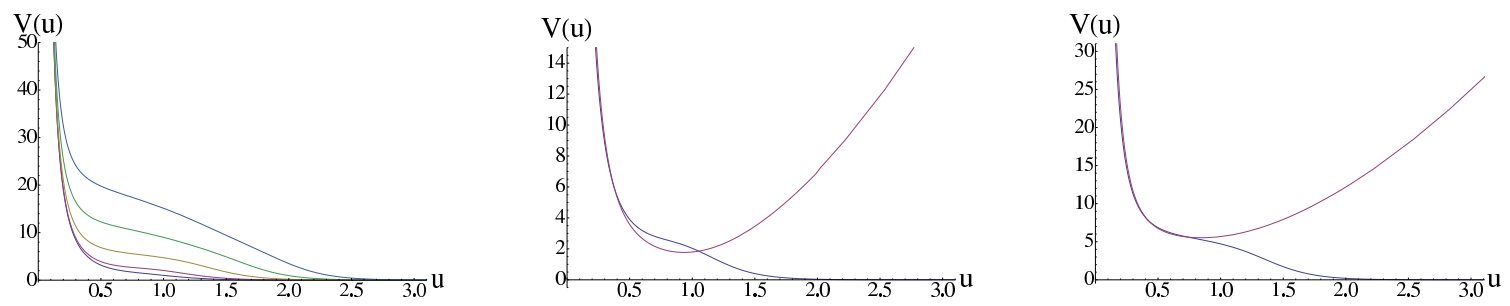

Figure 15. The Schrödinger potentials associated to the vector excitation in the deconfined phase, at zero momentum, for different values of $c_{1} \sim m_{q} / T$. In the first plot $c_{1}=0.01,1,2,3,4$. The second and third plot (respectively $c_{1}=1,2$ ) make a comparison with the potentials in the confined phase for the same values of $c_{1}$.

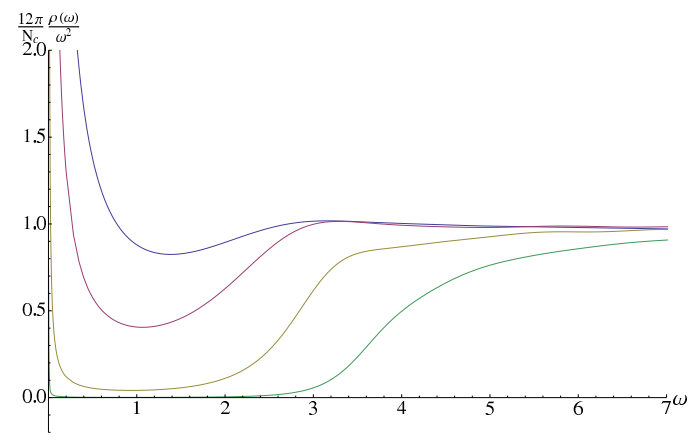

Figure 16. The spectral function (divided by $\omega^{2}$ ) in units of $\frac{N_{c}}{12 \pi}$ for $c_{1}=0.01,1,2,3,4$, from top to bottom.

what one has to do is to impose ingoing boundary conditions at the horizon $\left(\alpha(u) \sim e^{i \omega u}\right.$ near $u=\infty)^{8}$ and find the behaviour of the wavefunction near the boundary, namely compute $b_{1}, b_{2}$ matching the numerical result to the UV-expansion (4.12). Then, the spectral function is given by:

$$
\rho(\omega)=-\operatorname{Im} G_{R}(\omega)=\frac{N_{c}}{3 \pi^{2}} \operatorname{Im}\left(\frac{b_{2}}{b_{1}}\right)
$$

where we have replaced the parameters of our model by $\frac{N_{c}}{3 \pi^{2}}$, using (4.20). We show in figure 16 some examples of this computation. We have plotted $\frac{12 \pi}{N_{c}} \frac{\rho(\omega)}{\omega^{2}}$ in terms of $\omega$ for various values of $c_{1}$.

In [57], a bottom-up holographic model was built in order to discuss the physics of the $J / \Psi$ above the phase transition (see also [58] for related work). In the model of [57], there is also no discrete spectrum but, for low enough temperature, by engineering the potential, the $J / \Psi$ shows up as a peak in the spectral function (or, alternatively, as a small negative imaginary part of the associated quasinormal frequency). Namely, it has quasi-particle behaviour.

We now discuss the results comparing to [57]. The authors of [57] showed how the Schrödinger potential should look like to find some qualitative physical properties of the $J / \Psi$ meson. In our model, the potentials are found dynamically, and look similar to [57]

\footnotetext{
${ }^{8}$ Even if we use the notation with $u$ since it is better for illustrative purposes, we have found easier to perform the numerical computations in the $z$-coordinate.
} 
except for the important fact that they fail to have the dip of [57]. As a consequence, the $J / \Psi$ decay constant is too low (see figure 9 ) and a clear peak in the spectral function is absent. One may compare our figure 16 to figure 3 in [57].

It does not seem possible to introduce the dip feature in our model without inserting at least a new scale in the problem. Notice that the $\Lambda_{Q C D}$ does not play any role in the deconfined case for the present model, apart from setting the value of $T$ at which the transition takes place. In other words, the $z_{\Lambda}$ does not appear at all in the deconfined background. We speculate that one should modify the background by including some dependence on $\Lambda_{Q C D}$ in the deconfined phase in order to improve the model in these respects. It would be interesting to investigate whether analyzing the tachyon action in the less simple but well motivated backgrounds of "improved holographic QCD" models [47] might ameliorate the issue along this path.

\section{Discussion}

\subsection{Summary and general comments on the model}

In this paper, we have analyzed in detail several issues of the holographic model presented in [23]. The main ingredient is to describe the open string (meson) physics by using tachyon condensation, captured by Sen's action [25], as first advocated in [24]. The "tachyon", namely the lowest lying bifundamental scalar of the brane-antibrane system, is dual to the quark mass scalar bilinear operator and its condensation corresponds to chiral symmetry breaking. In order to make explicit computations, it is necessary to give an explicit form of the tachyon potential and to choose a curved gravitational background. We have considered extremely simple possibilities for both, see (3.4) and (2.2).

As we have already remarked, the model is inspired by string theory but is phenomenological. It does not provide a well controlled approximation to string theory in any limit. In this sense, it should be considered a bottom-up model. Nevertheless, the main point we want to make in this work is that it can be very useful to incorporate top-down derived ingredients as (3.1) into bottom-up models. We will make a comparison between qualitative properties of our model and the soft wall model [22] and modifications thereof. Successes of [22] include a reasonable qualitative and quantitative matching of properties of the measured mesonic states, including the Gell-Mann-Oakes-Renner relation and the Regge trajectories. These are also found in our model, together with the following extra appealing properties, which are automatic in our general set-up:

- The model incorporates confinement in the sense that the quark-antiquark potential computed with the usual AdS/CFT prescription [63] confines. Moreover, magnetic quarks are screened. The background solution stems from a gravitational action, that allows, for instance, to compute thermodynamical quantities. All of this are properties associated to the background geometry and were already discussed in [29].

- The string theory nature of the bulk fields dual to the quark bilinear currents is readily identified: they are low-lying modes living in a brane-antibrane pair. 
- Chiral symmetry breaking is realized dynamically and consistently, because of the tachyon dynamics. See [61, 62] for discussion and possible solutions in the soft-wall model context.

- In the present model, the mass of the $\rho$-meson grows with increasing quark mass, or, more physically, with increasing pion mass. This welcome physical feature is absent in the soft wall model, [22]. It occurs here because the tachyon potential multiplies the full action and in particular the kinetic terms for the gauge fields, which therefore couple to the chiral symmetry breaking vev. In our previous work [23], we exploited this fact in order to fit the strange-strange mesons together with the lightlight mesons, with rather successful results. In [64], the authors added the strange quark mass to the hard wall model and computed the dependence of vector masses on the quark mass. In that case however, this dependence of the vector masses originated only from the non-abelian structure and therefore misses at least part of the physics. ${ }^{9}$

- The soft wall requires assuming a quadratic dilaton in the closed string theory background. It has been shown that such a quadratic dilaton behaviour can never be derived from a gravitational action while keeping the geometry to be that of AdS. ${ }^{10}$ That the background is not found as a solution is a shortcoming if for instance one wants to study the thermodynamics of the underlying glue theory. The thermodynamics of the soft wall model is therefore ill-defined. In the present model, we found the background as a solution of a two-derivative approximation to non-critical string theory, see section 2. In order to obtain Regge behaviour, we also needed a further assumption: that the tachyon potential is asymptotically gaussian. However, this is rather natural since this potential has appeared in the literature, for instance [36, 37]. Still, we warn the reader that the formalism of $[36,37]$ cannot be directly and controllably connected to the present setup.

- Considering that the dynamics is controlled by a tachyon world-volume action automatically provides the model with a WZ term of the form given in [37, 66, 67]. We have not discussed this term at all in this work, but in [24] it was shown that properties like discrete symmetries (parity and charge conjugation) and anomalies are, in general, correctly described by analyzing this term.

In summary, we regard our model as being in the general framework of [22], but with several qualitative improvements due to the dynamics built in the action (3.1). Moreover, our starting assumptions are rather simple and well motivated from a top-down perspective, such that the ad hoc input is scarce. It is also encouraging to find that quantitative fits

\footnotetext{
${ }^{9}$ On the other hand, the quark mass dependence of the $\rho$-meson can be seen in different top-down models, see [65] for a recent work in the context of the Klebanov-Strassler model.

${ }^{10}$ This was shown in the second reference of $[12-15]$. In $[10,11]$ such behavior can be implemented for glue, but the metric changes appropriately, an important ingredient for implementing confinement in the glue sector.
} 
to experimental data are reasonably good, see [23] and section 4.5, but those are not the main aim of the present work.

We have also found several aspects in which the model does not capture features which are known from perturbative QCD.

The main issue is the leading large $q^{2}$ contribution to $\Pi_{V}\left(q^{2}\right)-\Pi_{A}\left(q^{2}\right)$ as will be discussed in section 6.3. We have also seen that our model does not work that well for large quark masses ${ }^{11}$ as it would grossly underestimate the decay constants for charmonium and no clear peaks are observed in the corresponding spectral functions in the deconfined phase (see the discussion of section 5). It would be interesting to know whether mild corrections of the model could ameliorate these issues or whether these are unsurmountable differences of models of this class to actual QCD physics.

The behaviour of $\frac{d\langle\bar{q} q\rangle}{d m_{q}}$ for small $m_{q}$ (figure 2) has been investigated in earlier classic works [68-70]. However, the leading IR divergence-free correction, is of order $1 / N^{2}$ at large $\mathrm{N}$, originating from a pion loop, [71, 72]. The leading- $\mathrm{N}$ corrections come from the four-derivative terms and are dominated by the scalar meson contribution. The behavior is qualitatively similar to what we find. For large $m_{q}$ we have not been able to calculate the asymptotic behavior but generically speaking we do not expect it to necessarily match that of QCD, as discussed in [68-70]. The reason is that the UV asymptotics of the bulk gravity solution are not necessarily the same as in QCD.

\subsection{Comments on effective actions for the open string tachyon}

The notion that a scalar bifundamental in a brane-antibrane system should be the holographic dual of QCD-like chiral symmetry breaking is rather simple and robust, see for instance [31, 75-78].

What is not obvious, however, is which effective action is best in order to describe a brane-antibrane system if curved spacetime. We have used the simple proposal of [25], but one should keep in mind that other alternatives might also be useful. We provide here a short guide to the literature on the issue.

Garousi and collaborators, starting from the early work [79], have tried to use explicit string theory computations in order to constrain the tachyon generalization of the DBI and WZ actions [80-82], see also [83]. In [38], an action for a $\mathrm{D} p-\overline{\mathrm{D}} p$ system based on a particular symmetriced trace prescription was proposed. ${ }^{12}$ There are subtle differences between the proposal of [38] and the one by Sen [25], which may have dramatic consequences. In fact, we have checked that using the symmetrized trace action of [38] for our model, one still finds Regge trajectories for vector and axial mesons but the slope for the axials changes,

\footnotetext{
${ }^{11}$ Perhaps this is not surprising since for heavy quarkonium perturbative methods and in particular nonrelativistic QCD (see $[73,74]$ for reviews) are accurate and it may be naive to expect that a dual theory can be a good approximation to the physics.

${ }^{12}$ In [38], a trace is needed even for a single brane-antibrane pair since the degrees of freedom are $2 \times 2$ matrices. In order to non-abelianize the flavor group in our case, one should also deal with the problem of how to implement traces on a non-abelian generalization of Sen's action (3.1). Investigating the physical consequences on the dual theory of this non-abelianization and of different proposals for the effective action [80-82] would be very interesting, but is beyond the scope of the present work.
} 
see appendix H. The study of other physical properties inferred from the action of [79] is beyond the scope of the present work.

In [84], it was discussed how to take into account the brane-antibrane distance in the string action. This is important for holographic duals, specially if one wants to insist in top-down approaches. A reason is that, in the weakly coupled picture, if one has brane and antibrane on top of each other in flat space, the tachyon would create a real instability (it cannot be compensated by the AdS curvature). Therefore one should think of separated branes as in the Sakai-Sugimoto model [19]. With this in mind, generalizations of [19], based on the action proposed in [84] where constructed in [85-87].

In a beautiful recent paper [88], building on the work [89], Niarchos proposed a different way of building the effective action which should better capture the physics of a separated brane-antibrane system. This may be useful in holographic modeling and in particular to improve the Sakai-Sugimoto model. We also refer the reader to [88] for a more exhaustive overview of the literature of tachyon effective actions.

\subsection{On the OPEs and the slope of the Regge trajectories}

There has been some debate in the literature on whether the large Euclidean momentum behaviour of two-point correlators can be used to constrain the behaviour of the QCD spectrum of excited mesons. The main point is to compare infinite sums like (4.21) (or, more precisely, differences of such sums: vector minus axial or scalar minus pseudoscalar) to the large $q^{2}$ behaviour expected from the operator product expansion (OPE). In particular, there is the question of whether different Regge slopes in the vector and axial (or scalar and pseudoscalar) channels

$$
\left(m_{n}^{V, A}\right)^{2} \sim \Lambda_{V, A}^{2} n+\text { const } \quad \text { for large } n
$$

are consistent with the OPEs. Notice that this is a theoretical question, irrespective of the experimental observation of the spectra. Let us give a brief and incomplete overview about the debate regarding this issue. For instance, in [90], a model with $\Lambda_{V} \neq \Lambda_{A}$ was put forward. Later, in [91], it was claimed that this model was inconsistent with the OPEs, but the arguments of [91] were called into question in [92], due to subtleties in the regularization of infinite sums like (4.21). More recently, works like [93-95] claim that the Regge slopes should be equal whereas the opposite conclusion was reached in [96-98].

We have found above that in our model, there are different asymptotic Regge slopes $\Lambda_{A}>\Lambda_{V}$. However, the coefficients of the leading logarithms in the large $q^{2}$ correlator for vectors and axials coincide, consistently. In order to illustrate this fact, let us remember that, asymptotically in the UV, our model resembles the soft wall of [22], see equation (4.15). In the soft wall model, the Regge slope is controlled by the constant $c$ of (4.15), but the quotient $F^{2} / \Lambda^{2}$ is independent of $c$ [99]. This quotient is indeed what controls the coefficient of the leading logarithm [92]. We thus have $\lim _{q^{2} \rightarrow \infty}\left(\Pi_{V}\left(q^{2}\right)-\Pi_{A}\left(q^{2}\right)\right)=0$ together with different Regge slopes. However, this is not enough to comply with the OPEs. The leading contribution to $\left(\Pi_{V}\left(q^{2}\right)-\Pi_{A}\left(q^{2}\right)\right)$ at large $q^{2}$ should be of order $q^{-4}$ because QCD does not have dimensionful quantities that allow to rewrite for instance a 
$q^{-2}$ term. We can resort to numerics to compute $\left(\Pi_{V}\left(q^{2}\right)-\Pi_{A}\left(q^{2}\right)\right)$ in our model and the result does not comply with the $q^{-4}$ expectation. This fails in the axial channel as shown in appendix F.

The obvious guess is that, since our holographic model is clearly not exactly QCD, it includes operators or condensates which are absent in QCD, modifying the subleading pieces of the correlators.

The same kind of problem is present for any holographic model we are aware of, see [99] for a discussion concerning the soft wall. ${ }^{13}$ However, this seems to us more of a technical problem that may be resolved by finding the appropriate potentials than a general obstruction to this class of models. Settling these issues requires further work.

\subsection{Outlook}

As we have discussed, our simple model is quite successful in describing many features of QCD. A lot of effort has been devoted in bottom-up models to estimate other QCD related properties as for example form factors, see for instance [100-108]. To reproduce such computations in the present setting and compare the results is an interesting problem that we leave open for the future. We have not studied non-trivial baryon number or chemical potentials, which would clearly be worthy extensions of the model.

There are some aspects of the present that would be worth improving, like the physics associated to heavy quarks (compared to the QCD scale). We have just explored the result of working with Sen's action in the simplest confining holographic background available in the literature $[28,29]$. Therefore, it is still left to understand the consequences of implementing the tachyon action in different backgrounds, as for instance those which go under the name of improved holographic QCD [10, 11, 47] or modifications thereof.

It could also be interesting to try to introduce the quarks beyond the quenched approximation and therefore compute the backreaction of the tachyon action for the fundamental fields on the gravity background. For a review of unquenched flavor in critical (ten-dimensional) string theory backgrounds, see [109].

Of course, it would be worth to provide a non-abelian generalization of this model, for which one should provide a technical prescription on how to take traces in the action. Another line of obvious interest would be to use the model for baryons. Since quark masses play a more dynamical role than in other bottom-up approaches, this could be interesting for the physics of the sigma-term.

Finally, and most importantly, we would like to point out that using an effective open string action like (3.1) in the framework of holography can well have interesting applications beyond the realm of strong interactions. For instance, many bottom-up technicolor models have appeared in the literature, see [110] for a review. It is a very interesting question to understand whether chiral symmetry breaking controlled by an action like (3.1) may offer new dynamical possibilities for the modeling of electroweak symmetry breaking. On the other hand, in the last years, many phenomenological models have been constructed in order to address some issues of superconductors and other condensed matter systems,

\footnotetext{
${ }^{13}$ A.P. thanks O. Cata for a discussion on this subject.
} 
for a review see [111]. Again, we would like to remark that (3.1) could hopefully lead to interesting new dynamics in different set-ups.

\section{Acknowledgments}

We would like to thank for useful conversations and correspondence. R. Casero, O. Cata, A. Cotrone, S. Eydelman, U. Gursoy, D. Mateos, V. Niarchos, F. Nitti, M. Panero, T. Pich, A. Pomarol, M. Shifman.

E. Kiritsis would like to thank CERN, ENS, ESI and Perimeter Institute for hospitality during the present work. The research of A.P is supported by grants FPA2007-66665C02-02 and DURSI 2009 SGR 168, and by the CPAN CSD2007-00042 project of the ConsoliderIngenio 2010 program. This work was partially supported by a European Union grant FP7-REGPOT-2008-1-CreteHEPCosmo-228644. I. Iatrakis was supported by an Onassis graduate fellowship.

\section{A Book-keeping summary of the parameters of the model}

We summarize here the parameters of the our model. There are two parameters coming from the background, the AdS radius $R$ and the position of the cigar tip $z_{\Lambda}$.

The action of the flavor brane-antibrane pair also includes $\alpha^{\prime}$ and two more parameters $g_{V}$ and $\lambda$ which are related to the normalization of the vector and tachyon fields respectively. The tachyon potential also includes two constants, $\mathcal{K}$ which is an overall factor in front of the action and $\mu^{2}$. It should also noticed that $\mu$ can be absorbed in $\tau(z)$ by redefining $\tilde{\tau} \rightarrow \mu \tau$. Then this parameter disappears from all equations. We used $\mu^{2}=\pi$, for the numerics through our analysis, but this does not affect any physical results of our model.

Another parameter which exists in the model is $c_{1}$ which appears in the UV asymptotic of the tachyon expectation value (3.7). $\quad c_{1}$ is proportional to the quark mass, with a proportionality constant $\beta$ which was introduced in (3.26), however $\beta$ does not appear in the equations for the spectrum or the decay constants, so its value is not relevant for the model predictions.

In total we have the following parameters $R, z_{\Lambda}, \alpha^{\prime}, g_{V}, \lambda, \mathcal{K}$ and $c_{1} . R, \alpha^{\prime}$ and $\lambda$ are related by equation (3.8), which relates the tachyon mass to the dimension of its dual operator. Then, we relate $g_{V}$ and $\lambda$ to the number of colors $N_{c}$ in QCD by matching the results of the vector and scalar two point functions as calculated in bulk on the one hand and in QCD on the other hand. The results are given in (4.20), (4.34), which relate $g_{V}$ to $\lambda$. Hence, finally the spectrum and the decay constants depend on $z_{\Lambda}, c_{1}$ and a combination of $R, g_{V}$ and $\alpha^{\prime}$ which was named $k$ and is given by, (4.26),

$$
k=\frac{4 R^{4} g_{V}^{4}}{3\left(2 \pi \alpha^{\prime}\right)^{2}}
$$




\section{B Analysis of singularities in the tachyon differential equation}

\section{B.1 Confining background}

In this appendix, we will investigate the existence of singular solutions of the tachyon equation of motion (3.9). It was argued already that $\tau$ can diverge only at the tip of the cigar. Therefore what is left to investigate is solutions where $\tau^{\prime}(z)$ diverges at a point $z_{0} \in\left[0, z_{\Lambda}\right]$, but $\tau(z)$ remains finite at the same $z_{0}$. We call these solutions "spurious". Taking into account $\tau^{\prime}(z) \gg \tau(z)$ in the neighborhood of $z_{0}$, the leading terms of eq. (3.9) are the first and the second one. Hence, the leading order equation is (we set $\mu=1$ in the sequel as it can be absorbed in the normalization of $\tau$ and $z_{\Lambda}=1$ )

$$
\tau^{\prime \prime}(z)-\frac{4}{3} z f(z) \tau(z)=0
$$

with solution in the vicinity of the divergence

$$
\tau^{\prime}(z)=\frac{1}{\sqrt{g(z)}}=\frac{1}{\sqrt{C-\frac{4}{3} z^{2}\left(1-\frac{2}{7} z^{5}\right)}} \quad, \quad \tau(z)=\int_{0}^{z} \frac{d z}{\sqrt{g(z)}}+\tau_{0}
$$

where $g(z)=C-\frac{4}{3} z^{2}\left(1-\frac{2}{7} z^{5}\right)$. The function $g(z)$ has either one or three real roots. In particular, there are the following three cases

1. $\mathbf{C}<\mathbf{0}$ : There are no roots of $g(z)$ in the interval $[0,1]$, since the only one root is at $z_{0}>1$. It should also pointed out that for $z \in[0,1], g(z)$ is negative so the solution does not exist.

2. $\mathbf{0}<\mathbf{C}<\frac{\mathbf{2 0}}{\mathbf{2 1}}$ : There is a single real zero at $z_{0} \in[0,1]$. While, the other two real zeros lie outside that interval.

3. When $\mathbf{C}=\frac{\mathbf{2 0}}{\mathbf{2 1}}$ the divergence happens exactly at the tip of the cigar.

4. $\mathbf{C}>\frac{\mathbf{2 0}}{\mathbf{2 1}}$ : Again there is no real zero in $\left[0, z_{0}\right]$.

If $g(z)$ has a real root $z_{0} \in[0,1]$, then it follows from (B.2) that $\tau^{\prime}(z)$ diverges at $z=z_{0}$, but $\tau(z)$ is regular there. Only in case that $z_{0}$ is a double root of $g(z)$, both $\tau(z)$ and $\tau^{\prime}(z)$ diverge at the same point. We are particularly interested in the above case where the acceptable solution diverges at some point $z_{0}$ in order to obtain the effect of chiral symmetry breaking in the dual quantum field theory. This is only managed if we tune the initial conditions ( $C$ here). There are two possibilities which lead to a double root of $g(z)$, in the context of the above approximation

1. $\mathbf{C}=\mathbf{0}$ : In that case the double root is at $z_{0}=0$. Then, eq. (B.1) has not real solutions, so it is not considered here.

2. $\mathbf{C}=\frac{\mathbf{2 0}}{\mathbf{2 1}}$ : The double root now is at $z=1$. 
The only rigorous result of the aforementioned analysis is that "spurious" singularities are generic if $0<C<\frac{20}{21}$.

If the tachyon diverges as a power law $\tau \sim\left(z-z_{0}\right)^{-a}$ with $a>0, \frac{\tau^{\prime}}{\tau} \sim \frac{1}{z-z_{0}}$ and the approximation described above is still valid, provided $z_{0} \neq 1$. But then, this is not a valid solution since the solution in the above approximation is of the form (B.2). This excludes such a divergence if $z_{0} \neq 1$.

The only other option of divergence of $\tau(z)$, and/or $\tau^{\prime}(z)$ at a point $z_{0} \in[0,1]$ is the case where $\tau^{\prime 2} \tau$ term is dominating. The relevant equation then is

$$
\tau^{\prime \prime}(z)+\tau^{\prime}(z)^{2} \tau=0
$$

which leads to

$$
\tau^{\prime}(z)=C e^{-\frac{1}{2} \tau(z)^{2}}
$$

For $\tau(z) \gg 1$, an approximate solution to eq. (B.3) is

$$
\frac{1}{\tau(z)} e^{\frac{1}{2} \tau(z)^{2}} \simeq C z+\ldots
$$

Therefore, $\tau(z)$ diverges only if $z \rightarrow \infty$ which is not allowed in the present geometry as $z \in[0,1]$. Hence this case is excluded.

From the above mentioned, we conclude that the only place where $\tau(z)$ diverges is at $z=1$. In order to find the solution of diverging $\tau(z)$ we must tune the initial conditions in the UV, see section 3. We also showed that "spurious" singularities in the interior of the interval $[0,1]$ are generic for a range of initial conditions.

The existence of "spurious" singularities has been verified numerically, and it fits the asymptotics (B.2). An example of this behavior is shown in figure 17. We solve numerically eq. (3.9) for arbitrary initial conditions, meaning that the mass and the vev are not tuned according to the plot in figure 2 . In particular, we have chosen $c_{1}=0.1, c_{3}=0.439$. In this case we notice that $\tau^{\prime}\left(z_{0}\right) \gg \tau\left(z_{0}\right)$ at $z_{0}=0.8696<1$. The right part of figure 17 includes the plot of the derivative of the numerical solution (red line) and the expression for $\tau^{\prime}(z)$ given in eq. (B.2) (dashed blue line), near $z_{0}$. On the left part we have plotted $\tau(z)$ and the asymptotic solution (B.2) (blue dots). The parameters of the expressions in (B.2) are $C=2.71758<\frac{20}{21} \pi$ and $\tau_{0}=-0.28$. For those values the asymptotic solution (B.2) fits the numerical solution of the full equation near $z_{0}$.

\section{B.2 Deconfined background}

We now look for singular solutions of the tachyon equation of motion in the deconfined background, eq. (3.17). Considering again that $\tau^{\prime}(z) \gg \tau(z)$ at the vicinity of $z_{0}$, with $z_{0}$ being the point where $\tau^{\prime}\left(z_{0}\right) \rightarrow \infty$, the leading terms of eq. (3.17) near $z_{0}$ are

$$
\tau^{\prime \prime}(z)+\frac{z^{2}}{3} f(z)\left(-\frac{4}{z}+\frac{f^{\prime}(z)}{2 f(z)}\right) \tau^{\prime}(z)^{3}=0
$$

where $f(z)=1-\frac{z^{5}}{z_{T}^{5}}$. The solution reads 

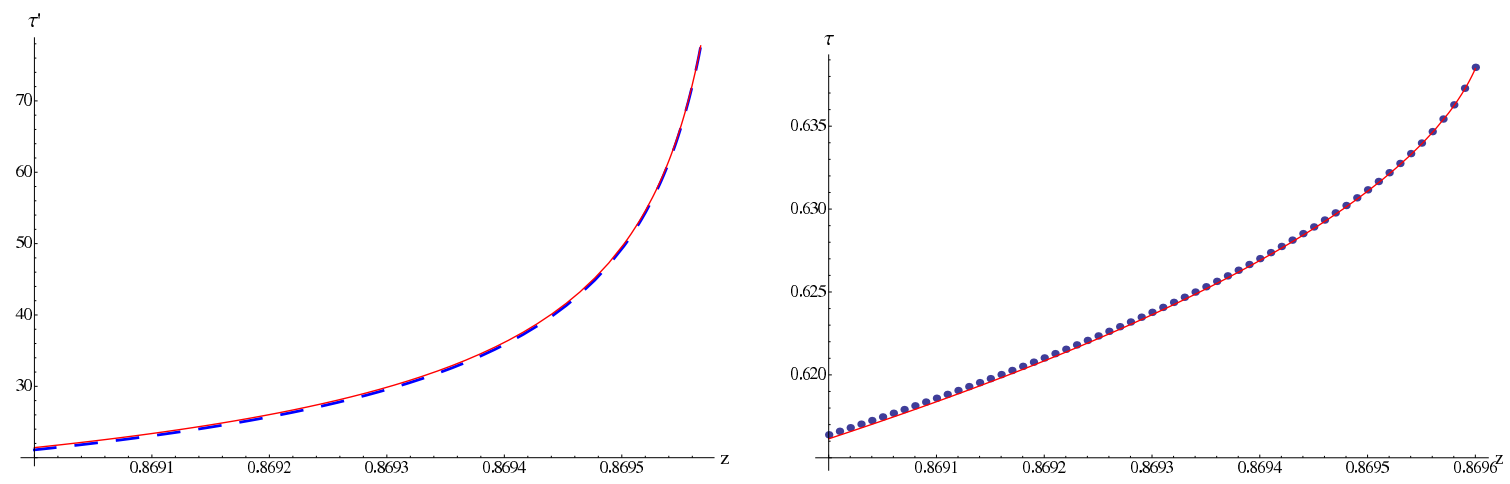

Figure 17. An example of a solution of type which diverges at $z_{0}=0.8696<1$ was found numerically. The numerical solution and its derivative are compared to the asymptotic solution

$$
\tau(z)=\int_{0}^{z} \frac{d z}{\sqrt{C-\frac{4}{3} z^{2}\left(1-\frac{3}{28} \frac{z^{5}}{z_{T}^{5}}\right)}}+\tau_{0}
$$

The function $g(z)=C-\frac{4}{3} z^{2}\left(1-\frac{3}{28} \frac{z^{5}}{z_{T}^{5}}\right)$ has a maximum in $z=0$ and no other extrema in the interval $\left[0, z_{T}\right]$. If this function is zero at some point $z_{0}$, then $\tau^{\prime}\left(z_{0}\right)$ is infinite and $\tau\left(z_{0}\right)$ is finite except if $g\left(z_{0}\right)=0$ has a double root. Similarly to the confining background case, there are three choices

1. $\mathbf{C}<\mathbf{0}: g(z)$ is not zero in the interval $\left[0, z_{T}\right]$.

2. $\mathbf{0}<\mathbf{C}<\frac{\mathbf{2 5}}{\mathbf{2 1}} \mathbf{z}_{\mathbf{T}}^{\mathbf{2}}: g(z)$ has one real root in the interval $\left[0, z_{T}\right]$ which is not a double root. When, $C=\frac{25}{21}$ the root of $g(z)$ is at $z_{0}=1$.

3. $\mathbf{C}>\frac{\mathbf{2 5}}{\mathbf{2 1}} \mathbf{z}_{\mathbf{T}}^{\mathbf{2}}$ : There is no root of $g(z)$ in $\left[0, z_{T}\right]$.

So, for a suitable range of the initial conditions $(C)$ we may have a solution with diverging $\tau^{\prime}(z)$ and finite $\tau(z)$ at some point $z_{0} \in[0,1]$. A double root is possible to be found in case of $C=0$, and it is at $z=0$.

The discussion about the case where the term $\tau^{\prime 2} \tau$ of eq. (3.17) is leading, remains the same as the one that follows eq. (B.3) in the previous appendix. Therefore, in case of the deconfined background tachyon cannot diverge in the interval $[0,1]$ but "spurious" singularities of the form $\tau^{\prime}(z) \gg \tau(z)$ exist in general.

\section{Scheme dependence of the condensate and the constant $\alpha$}

We have introduced an arbitrary constant $\alpha$ in (3.24) associated to a counterterm that gives a finite contribution and therefore cannot be fixed by demanding the cancellation of divergences. This is a common situation in holographic renormalization and is related to scheme dependence of renormalization in the field theory side, as we now discuss.

The gauge invariant composite operator $q_{L}^{\dagger} q_{R}$ must be defined by a subtraction in QFT. To asses what enters in such subtractions we must study the OPE $q_{L}^{\dagger}(x) q_{R}(y)$ as $x \rightarrow y$. 
The operator can then be defined by point splitting, subtracting divergent contributions, and then taking the limit $x=y$.

Apart from the identity operator, all other operators that can appear in the OPE $q_{L}^{\dagger}(x) q_{R}(y)$ do not provide divergences. These include the operator itself : $q_{L}^{\dagger}(x) q_{R}(x)$ : as well higher dimension operators. Therefore this composite operator can be defined by normal ordering. To make this precise we use a Dirac basis, so that we have the real part $\bar{q}(x) q(y)$ and the imaginary part $\bar{q}(x) \gamma^{5} q(y)$. The imaginary part is finite and no subtraction is needed. For the real part we introduce a momentum cutoff $\Lambda$ as we will be working in momentum space. We will therefore define

$$
: \bar{q} q:=\lim _{\Lambda \rightarrow \infty}\left[\bar{q} q-a_{1} \Lambda^{3}-a_{2} m \Lambda^{2}-a_{3} m^{2} \Lambda-a_{4} m^{3} \log \frac{\Lambda^{2}}{m^{2}}-a_{5} m^{3}\right]
$$

where on the left-hand side we subtracted the most general expression of scaling dimension 3. This should be enough in a conformally invariant theory. If the theory is asymptotically free, as in QCD, more subtractions are necessary, as there is one more relevant scale entering the problem, namely $\Lambda_{Q C D}$. However in the model we consider in this paper, the physics in the UV is conformal so these subtractions are enough.

To establish the coefficients in (C.1) we must require that the (perturbative, short distance) part of the vev is finite when we remove the cutoff. We have

$$
\langle: \bar{q} q:\rangle=\lim _{\Lambda \rightarrow \infty}\left[\langle\bar{q} q\rangle-a_{1} \Lambda^{3}-a_{2} m \Lambda^{2}-a_{3} m^{2} \Lambda-a_{4} m^{3} \log \frac{\Lambda^{2}}{m^{2}}-a_{5} m^{3}\right]
$$

We calculate (in Euclidean space)

$$
\begin{aligned}
\langle\bar{q} q\rangle & =-N_{c} \operatorname{Tr} \int \frac{d^{4} p}{(2 \pi)^{4}} \frac{-i \not q+m}{q^{2}+m^{2}}=-4 N_{c} m \int \frac{d^{4} p}{(2 \pi)^{4}} \frac{1}{q^{2}+m^{2}} \\
& =-2 N_{c} \Omega_{3} m \int_{0}^{\Lambda^{2}} \frac{p^{2} d p^{2}}{p^{2}+m^{2}}=-2 N_{c} \Omega_{3} m\left[\Lambda^{2}-m^{2} \log \frac{\Lambda^{2}+m^{2}}{m^{2}}\right] \\
& =-2 N_{c} \Omega_{3} m\left[\Lambda^{2}-m^{2} \log \frac{\Lambda^{2}}{m^{2}}+\mathcal{O}\left(\Lambda^{-2}\right)\right]
\end{aligned}
$$

where $\Omega_{3}$ is the volume of th unit 3 -sphere.

To renormalize we must choose

$$
a_{1}=0 \quad, \quad a_{2}=-2 N_{c} \Omega_{3}=-a_{4} \quad, \quad a_{3}=0
$$

while $a_{5}$ can be arbitrary. It is this arbitrariness that is reflected in the coefficient $\alpha$ in the holographic renormalization setup in (3.27). ${ }^{14}$

In some cases, this scheme dependence can be fixed on physical grounds. For instance, in supersymmetric cases, one can demand that the renormalized on-shell action is zero, see [112] for an example. We could not find any convincing prescription to fix $\alpha$ in the

\footnotetext{
${ }^{14}$ In a theory like QCD, the arbitrariness involves the addition of a finite function $m^{3} f\left(\frac{m}{\Lambda_{Q C D}}\right)$ that reflects the presence of an extra mass scale.
} 
present case. A possibility would be to demand that the condensate vanishes for asymptotically large quark masses, but we have checked numerically that it is not possible. This is not surprising, since we have seen that our model works much better for small masses than for large ones.

\section{Converting to Schrödinger form}

In many cases, it is useful to write down the Sturm-Liouville problem which determines the spectrum of any given mode as a Schrödinger-like equation. Let us start by writing a generic quadratic five-dimensional action for a field $\Psi\left(x^{\mu}, z\right)$ :

$$
S=-\frac{1}{2} \mathcal{K}_{\Psi} \int d^{4} x d z\left(A(z)\left(\partial_{z} \Psi\right)^{2}+B(z) \eta^{\mu \nu} \partial_{\mu} \Psi \partial_{\nu} \Psi+C(z) \Psi \partial_{z} \Psi+M(z) \Psi^{2}\right),
$$

where we have allowed an arbitrary constant multiplying the action. Let us consider $\Psi=e^{i q x} \psi(z)$ and define as $m_{n}^{2}$ the discrete set of values of $-q^{2}$ which satisfy the appropriate normalizability conditions of the Sturm-Liouville problem. The discrete set of solutions satisfy the equation of motion extracted from (D.1):

$$
-\frac{1}{B(z)} \partial_{z}\left(A(z) \partial_{z} \psi_{n}(z)\right)+h(z) \psi_{n}(z)=m_{n}^{2} \psi_{n}(z)
$$

where we have introduced:

$$
h(z) \equiv \frac{1}{B(z)}\left(M(z)-\frac{1}{2} \partial_{z} C(z)\right) .
$$

We can define the orthonormality condition:

$$
\int d z B(z) \psi_{n}(z) \psi_{m}(z)=\delta_{m n}
$$

We now define a new radial variable $u$, and a rescaled field $\alpha$ in terms of a function $\Xi$ as:

$$
d u=\sqrt{\frac{B(z)}{A(z)}} d z, \quad \alpha=\Xi \psi, \quad \Xi=(A(z) B(z))^{\frac{1}{4}},
$$

The Sturm-Liouville problem now takes the Schrödinger form:

$$
-\frac{d^{2} \alpha_{n}(u)}{d u^{2}}+V(u) \alpha_{n}(u)=m_{n}^{2} \alpha_{n}(u)
$$

where the Schrödinger potential is:

$$
V(u)=\frac{1}{\Xi} \frac{d^{2} \Xi}{d u^{2}}+h(u)
$$

Substituting (D.4) in (D.5), we find that in the new variables, the normalization condition is the canonical one:

$$
\int d u \alpha_{n}(u) \alpha_{m}(u)=\delta_{m n}
$$

In order to estimate the mass of the modes with large eigenvalues, it is sometimes useful to employ a WKB formula:

$$
\frac{d\left(m_{n}^{2}\right)}{d n}=2 \pi\left[\int_{u_{1}}^{u_{2}} \frac{d u}{\sqrt{m_{n}^{2}-V(u)}}\right]^{-1}
$$

where $u_{1}$ and $u_{2}$ are the classical turning points. 


\section{E Two-point function and sum rules}

We describe here how, typically, the bulk solution needed to compute a two-point correlator from the gravity side can be written in terms of an infinite sum. Physically, two-point functions at arbitrary momenta are expressed as a sum over the discrete set of physical states. This is not a new result but, however, we believe that explicitly making the discussion as shown below can be illustrative. We remark that the argument of this appendix is not general in the sense that we have not included in the reasoning the possibility of having counterterms or other subtleties which may to be dealt with in a case by case basis.

Let us start with the equation:

$$
-\frac{1}{B(z)} \partial_{z}\left(A(z) \partial_{z} \psi(z)\right)+h(z) \psi(z)=-q^{2} \psi(z)
$$

where we have used the notation of appendix D. We want to find a solution $\psi_{q}(z)$ such that in the boundary $\psi_{q}(0)=1$, and which is IR-normalizable. Our goal is to write $\psi_{q}(z)$ in terms of the discrete infinite set of solutions $\psi_{n}(z)$ of the Sturm-Liouville problem (D.2), normalized as (D.4). Let us momentarily change to the notation with $\alpha(u)$, in which the problem is converted to:

$$
-\frac{d^{2} \alpha(u)}{d u^{2}}+V(u) \alpha(u)=-q^{2} \alpha(u)
$$

and the discrete spectrum is $\alpha_{n}(u)$ with (D.8) as normalization and the completeness relation:

$$
\sum_{n} \alpha_{n}(u) \alpha_{n}\left(u^{\prime}\right)=\delta\left(u-u^{\prime}\right)
$$

We introduce:

$$
G\left(u, u^{\prime}\right)=-\sum_{n} \frac{\alpha_{n}(u) \alpha_{n}\left(u^{\prime}\right)}{q^{2}+m_{n}^{2}}
$$

such that it is a Green function, $\left[\frac{d^{2}}{d u^{2}}-V(u)-q^{2}\right] G\left(u, u^{\prime}\right)=\delta\left(u-u^{\prime}\right)$. Let us assume now that the UV boundary is at $u=0$ and that UV-normalizability implies that $\alpha_{n}(0)=0$, such that $G\left(0, u^{\prime}\right)=0$. Regarding (D.5), $\alpha_{q}=\Xi \psi_{q}$, such that for generic momentum the $\mathrm{UV}$ condition is $\alpha_{q}(0)=\Xi(0)$. The solution we are looking for reads:

$$
\alpha_{q}(u)=\Xi(u)+\int_{0}^{\infty} G\left(u, u^{\prime}\right)\left(h\left(u^{\prime}\right)+q^{2} \Xi\left(u^{\prime}\right)\right) d u^{\prime}
$$

We can translate this back to the original variables. After some manipulations, we get our final result:

$$
\psi_{q}(z)=1-\sum_{n} \frac{\psi_{n}(z)}{m_{n}^{2}} \int_{0}^{\infty} \frac{h\left(z^{\prime}\right) B\left(z^{\prime}\right)}{\Xi\left(z^{\prime}\right)} \psi_{n}\left(z^{\prime}\right) d z^{\prime}-q^{2} A(0) \sum_{n} \frac{\psi_{n}(z) \psi_{n}(0)}{m_{n}^{2}\left(q^{2}+m_{n}^{2}\right)}
$$

Two-point correlators are built from the on-shell action associated to this solution, which can be typically found by computing the derivatives fo $\psi_{q}(z)$ at the boundary. From the last term in (E.6) one can find the decay constants of the states in the spectrum, as in section 4.1.3. The second term is $q$-independent and in fact it can be thought of as the $q^{2}=0$ contribution. For the axial excitation, this is related to the pion decay constant whereas for the vector excitation, this term is absent since $h(z)=0$ in that case. 


\section{F Axial current-current correlator}

The axial current-current correlator is now derived following the same procedure as in section (4.1.2). We are interested in the two point function in the limit of large Euclidean momenta. We expect that the leading term will be the same as in the vector current correlator, but the subleading term is different.

We define the correlator as

$$
\int_{x} e^{i q x}<J_{\mu}(x) J_{\nu}(0)>=\left(q^{2} \eta_{\mu \nu}-q_{\mu} q_{\nu}\right) \Pi_{A}\left(q^{2}\right)
$$

This is calculated by differentiating twice the on-shell bulk action. Integrating by parts (4.24) we find

$$
S_{A, \text { reg }}=\frac{\left(2 \pi \alpha^{\prime}\right)^{2}}{g_{V}^{4}} \mathcal{K} \int \frac{d^{4} q}{(2 \pi)^{4}}\left(e^{-\frac{1}{2} \mu^{2} \tau^{2}} g_{x x} \tilde{g}_{z z^{-1}}^{-\frac{1}{2}} A_{\mu}(q, z) \partial_{z} A^{\mu}(-q, z)\right)_{z=\epsilon}
$$

where $A_{\mu}(q, z)=\psi^{A}(q, z) A_{0}(q)$. Then, we insert the asymptotic solution (4.28) into the action

$$
S_{A}=\frac{\left(2 \pi \alpha^{\prime}\right)^{2}}{g_{V}^{4}} \mathcal{K} R \int \frac{d^{4} q}{(2 \pi)^{4}} A_{\mu}^{0}(q) A_{0}^{\mu}(-q)\left(2 b_{2}(q)+\left(q^{2}+k \mu^{2} c_{1}^{2}\right)\left(\frac{1}{2}+\log \epsilon\right)\right)
$$

where we have set $b_{1}=1$. The last term is cancelled by the corresponding counterterm from (4.4), so after differentiating twice with respect to $A_{0}(q)$ we find the final answer

$$
\Pi_{A}\left(q^{2}\right)=-4 \frac{\mathcal{K} R\left(2 \pi \alpha^{\prime}\right)^{2}}{g_{V}^{4}} \frac{b_{2}(q)}{q^{2}}
$$

We now compute $b_{2}(q)$ for large $q^{2}$, similarly with section (4.1.2). We convert (4.25) to Schrödinger form. Then, the new variable $u$ reads

$$
u \simeq z, \quad u \simeq \frac{z_{\Lambda}}{\sqrt{3}} \mu \tau
$$

in the UV and IR respectively. We calculate the asymptotic behavior of the Schrödinger potential in the UV and IR

$$
V_{U V} \simeq \frac{3}{4 u^{2}}+k \mu^{2} c_{1}^{2}, \quad V_{I R} \simeq c^{2} u^{2}
$$

where $c^{2}=\frac{1}{z_{\Lambda}^{4}}\left(\frac{9}{4}+3 k\right)$. Adding these two contributions we finally find the equation of motion the axial modes

$$
-\partial_{u}^{2} \alpha+\left(\frac{3}{4 u^{2}}+k \mu^{2} c_{1}^{2}+c^{2} u^{2}\right) \alpha+q^{2} \alpha=0
$$

where $\alpha(u) \simeq u^{-\frac{1}{2}} \psi^{A}(u)$ near $z=0$. Its general solution reads

$$
\alpha(u)=k_{1} \frac{e^{\frac{-c u^{2}}{2}}}{\sqrt{u}} U\left(\frac{q^{2}+k \mu^{2} c_{1}^{2}}{4 c}, 0, c u^{2}\right)+k_{2} \frac{e^{\frac{-c u^{2}}{2}}}{\sqrt{u}} L^{-1}\left(\frac{q^{2}+k \mu^{2} c_{1}^{2}}{4 c}, 0, c u^{2}\right),
$$


we set $k_{2}=0$, since the generalized Laguerre polynomial is going to infinity in the IR. $k_{1}$ is found such that $\lim _{z \rightarrow 0} \psi^{A}(q, z)=1$

$$
\psi^{A}=\frac{q^{2}+k \mu^{2} c_{1}^{2}}{4 c} \Gamma\left(\frac{q^{2}+k \mu^{2} c_{1}^{2}}{4 c}\right) e^{\frac{-c u^{2}}{2}} U\left(\frac{q^{2}+k \mu^{2} c_{1}^{2}}{4 c}, 0, c u^{2}\right)
$$

By expanding the solution for large momenta we find

$$
\lim _{q^{2} \rightarrow \infty} \frac{b_{2}}{q^{2}}=\frac{1}{4} \log \left(z_{\Lambda}^{2} q^{2}\right)-\frac{1}{4}(1+\log 4-2 \gamma)+\frac{k \mu^{2} c_{1}^{2}\left(2 \gamma-\log 4+\log q^{2}\right)}{q^{2}}+\frac{3 k^{2} \mu^{4} c_{1}^{4}-8 c^{2}}{24 q^{4}}+\ldots
$$

and eventually

$$
\begin{aligned}
& \Pi_{A}\left(q^{2}\right)=-\frac{\mathcal{K} R\left(2 \pi \alpha^{\prime}\right)^{2}}{g_{V}^{4}} \\
& \times\left(\log \left(z_{\Lambda}^{2} q^{2}\right)-(1+\log 4-2 \gamma)+4 \frac{k \mu^{2} c_{1}^{2}\left(2 \gamma-\log 4+\log q^{2}\right)}{q^{2}}+\frac{3 k^{2} \mu^{4} c_{1}^{4}-8 c^{2}}{6 q^{4}}+\ldots\right)
\end{aligned}
$$

We notice that for $c_{1}=0$ the result coincides with the vector current two point function. The $1 / q^{2}$ term, which is absent in the QCD result, comes from the mass term of the axial field $\left(\sim \tau^{2} A_{\mu} A^{\mu}\right)$, see (4.24).

\section{G Excitation equations in the deconfined phase}

We assemble here the the Shrödinger functions, as defined in appendix B, for the equations of motion of field excitation modes in the deconfined background (2.8), at vanishing spatial momentum. These modes satisfy an equation of the form (D.2) but there is no discrete spectrum.

In case of the vector excitations, we have already mentioned the functions giving rise to the Schrödinger potential approach in (5.1). Then, the variable $u$ reads

$$
u=\int_{0}^{z} \sqrt{\frac{\tilde{g}_{z z}(\tilde{z})}{g_{t t}(\tilde{z})}} d \tilde{z},
$$

which remains the same for all different excitations.

For axial-vector mesons $A(z)$ and $B(z)$ are the same as for the vectors (5.1), but now we also have:

$$
h(z)=\frac{M(z)}{B(z)}=k \mu^{2} \frac{\tau^{2}}{z^{2}} f_{T}(z) .
$$

The Schrödinger functions appearing in the equations of motion of scalar excitation modes are

$$
\begin{array}{ll}
A(z)=e^{-\frac{1}{2} \mu^{2} \tau^{2}} g_{x x}^{\frac{3}{2}} g_{t t}^{\frac{1}{2}} \frac{g_{z z}}{\tilde{g}_{z z}^{-\frac{3}{2}}}, & B(z)=e^{-\frac{1}{2} \mu^{2} \tau^{2}} g_{x x}^{\frac{3}{2}} g_{t t}^{-\frac{1}{2}} \frac{g_{z z}}{\tilde{g}_{z z}^{1 / 2}}, \\
C(z)=-2 \mu^{2} e^{-\frac{1}{2} \mu^{2} \tau^{2}} \frac{g_{x x}^{\frac{3}{2}} g_{t t}^{\frac{1}{2}}}{\tilde{g}_{z z}^{1 / 2}} \tau(z) \partial_{z} \tau(z), & M(z)=\frac{\mu^{2}}{2 \pi \alpha^{\prime} \lambda} e^{-\frac{1}{2} \mu^{2} \tau^{2}}\left(\mu^{2} \tau^{2}-1\right) g_{x x}^{\frac{3}{2}} g_{t t}^{\frac{1}{2}} \tilde{g}_{z z}^{\frac{1}{2}} .
\end{array}
$$


The function $M(z), B(z)$ and $C(z)$ combine to give a quite simple expression for the $h(z)$ defined in (D.3):

$$
h(z)=-\frac{3}{z^{2}} f_{T}(z)
$$

Finally, for the pseudoscalars we have

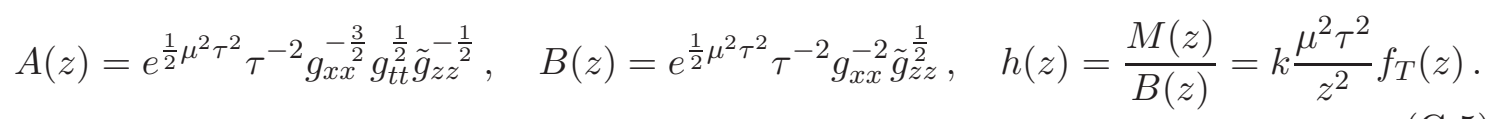

\section{H The action with the symmetric trace and Regge slopes}

In [38], Garousi proposed an effective action for the brane-antibrane system which has subtle difference with respect to Sen's one [25], which we have used in this work. One may wonder what would be the physical consequences of using such an action in our model. We focus in this appendix on the behaviour of the spectra of highly excited vectors and axial vectors. The equations for the vectors are not modified with respect to the main text. The equations for the axials are different. It turns out that they still obey a Regge law $m_{n}^{2} \propto n$ for large excitation number $n$ but with different slope compared to the main text. This slope is still larger than the one for vectors. We will not deal in the present work with other possible phenomenological implication of this different tachyon action.

Garousi's action reads: ${ }^{15}$

$$
S=-\mathrm{S} \operatorname{Tr} \int d^{4} x d z e^{-\hat{T}^{2}} \sqrt{-\operatorname{det}\left(g_{M N}+\hat{F}_{M N}+D_{M} \hat{T} D_{N} \hat{T}\right)}
$$

where hatted symbols are 2x2 matrices:

$$
\hat{T}=\left(\begin{array}{cc}
0 & T \\
T^{*} & 0
\end{array}\right), \quad \hat{F}_{M N}=\left(\begin{array}{cc}
F_{M N}^{(L)} & 0 \\
0 & F_{M N}^{(R)}
\end{array}\right), \quad D_{M} \hat{T}=\left(\begin{array}{cc}
0 & D_{M} T \\
\left(D_{M} T\right)^{*} & 0
\end{array}\right) .
$$

with $F_{M N}^{(i)}=\partial_{M} A_{N}^{(i)}-\partial_{N} A_{M}^{(i)}$ and $D_{M} T=\partial_{M} T+i\left(A_{M}^{(L)}-A_{M}^{(R)}\right) T=\partial_{M} T+2 i A_{M} T$ the usual field strength and covariant derivative, where the definition (4.1) has been substituted. The STr means that one has to symmetrice in $\hat{F}_{M N}, D_{M} \hat{T}, \hat{T}$ after expanding the square root, and then take the trace.

The expression (H.1) is quite involved but we will see that in the particular case we are interested, one can deal with it: we will consider quadratic excitations of the gauge fields, while the tachyon phase is set to a trivial constant and the tachyon modulus is a non-trivial $z$-dependent function (so we have to keep all orders in $\tau, \partial_{z} \tau$ ). We again take

\footnotetext{
${ }^{15}$ We adapt it to our present framework, for instance defining the covariant derivative with a different sign and disregarding the $B_{\mu \nu}$ field. With respect to the main text, we will fix the value of some of the constants that we have defined, namely $g_{V}^{2}=2 \pi \alpha^{\prime}, \lambda=1 /\left(2 \pi \alpha^{\prime}\right), \mathcal{K}=1, \mu^{2}=2$. Regarding (3.8), this implies $R^{2}=3 / 2$. This is inessential (the constants can be easily restored) and we have done it for the sake of clarity of the equations. Our convention will be that indices $M, N$ running over the five space-time coordinates are contracted with the metric $g_{M N}$ whereas indices $\mu, \nu$ running over the Minkowski directions are contracted with the flat metric $\eta_{\mu \nu}$.
} 
a gauge with $A_{z}^{(i)}=0$. This is enough to compute the vector and axial spectrum in the tachyon background.

So let us compute the quadratic expansion in gauge fields. There are terms in $A_{\mu}^{2}$ coming from the covariant derivatives and terms with $F^{(i) 2}$. In principle, there could be terms with, schematically, $i \tau \partial_{z} \tau A F$ coming from a DTDT F product, but these terms would make the action complex and are removed by the symmetric trace prescription. In the following, we make the computation in two steps: we first compute the $A_{\mu}^{2}$ terms and then compute the $F^{2}$ terms.

In order to compute the $A_{\mu}^{2}$ terms, we can consider the action:

$$
\begin{aligned}
S_{A^{2}} & =-\operatorname{STr} \int d^{4} x d z e^{-\hat{T}^{2}} \sqrt{-\operatorname{det}\left(g_{M N}+D_{M} \hat{T} D_{N} \hat{T}\right)}= \\
& =-\int d^{4} x d z \sqrt{-\operatorname{det} g} \operatorname{STr}\left[e^{-\hat{T}^{2}} \sqrt{\operatorname{det}\left(\delta_{N}^{M}+D^{M} \hat{T} D_{N} \hat{T}\right)}\right]
\end{aligned}
$$

We now compute the determinant. Being inside a $\mathrm{S} \operatorname{Tr}$, the $\hat{T}$ matrices can be considered as commuting objects, so $\sqrt{\operatorname{det}\left(\delta_{N}^{M}+D^{M} \hat{T} D_{N} \hat{T}\right)}=\sqrt{1+D^{M} \hat{T} D_{M} \hat{T}}$. We have to expand the square root. In order to simplify notation, let us define $s_{j}$, the coefficients of such expansion:

$$
\sqrt{1+\xi}=\sum_{j=0}^{\infty}(-1)^{j+1} \frac{(2 j-3) ! !}{j ! 2^{j}} \xi^{j} \equiv \sum_{j=0}^{\infty} s_{j} \xi^{j}
$$

Thus, also expanding the exponential:

$$
\mathcal{L}_{A^{2}}=-\sqrt{-\operatorname{det} g} \sum_{k=0}^{\infty} \frac{(-1)^{k}}{k !} \sum_{j=0}^{\infty} s_{j} \operatorname{STr}\left[\hat{T}^{2 k}\left(D^{M} \hat{T} D_{M} \hat{T}\right)^{j}\right]
$$

The next step is to perform the symmetriced trace, and a major simplification comes out because of the particular computation we are doing. Define:

$$
\hat{J}_{1}=\left(\begin{array}{ll}
0 & 1 \\
1 & 0
\end{array}\right), \quad \hat{J}_{2}=\left(\begin{array}{cc}
0 & -1 \\
1 & 0
\end{array}\right)
$$

such that (use $T=T^{*}=\tau$ ):

$$
\hat{T}=\tau \hat{J}_{1} \quad D_{M} \hat{T}=\partial_{M} \tau \hat{J}_{1}-2 i A_{M} \tau \hat{J}_{2}
$$

The order zero term in $A_{M}$, i.e. the action for the tachyon modulus is just

$$
\mathcal{L}_{\tau}=-2 \sqrt{-\operatorname{det} g} e^{-\tau^{2}} \sqrt{1+\partial^{M} \tau \partial_{M} \tau},
$$

the same used in the main text. This means that the discussion of section 3 still holds.

We now isolate the quadratic term in $A_{M}$. One out of the $j$ factors of $\left(D^{M} \hat{T} D_{M} \hat{T}\right)^{j}$ has to be $g_{x x}^{-1}\left(-2 i \tau \hat{J}_{2}\right)^{2} A_{\mu} A^{\mu}$ while the other $j-1$ factors are $\hat{J}_{1}^{2} g_{z z}^{-1}\left(\partial_{z} \tau\right)^{2}$ each. Notice there cannot be crossed terms because $\partial_{M} \tau A^{M}=0$ in the case we are conisdering. Thus:

$$
\mathcal{L}_{A^{2}}=-g_{x x}^{-1} \sqrt{-\operatorname{det} g} \sum_{k=0}^{\infty} \frac{(-1)^{k}}{k !} \sum_{j=0}^{\infty} s_{j} j \tau^{2 k}\left(g_{z z}^{-1}\left(\partial_{z} \tau\right)^{2}\right)^{j-1}\left(-4 A_{\mu} A^{\mu} \tau^{2}\right) \operatorname{STr}\left[\hat{J}_{1}^{2 k+2 j-2} \hat{J}_{2}^{2}\right]
$$


The $j$ at the beginning of the second line of course comes because the $A_{\mu} A^{\mu}$ term can be chosen from any of the $j$ factors in $\left(D^{M} \hat{T} D_{M} \hat{T}\right)^{j}$. In order to perform the symmetriced trace, notice that, in general:

$$
\mathrm{S} \operatorname{Tr}\left[\hat{J}_{1}^{2 n} \hat{J}_{2}^{2}\right]=\frac{1}{2 n+1}\left((n+1) \operatorname{Tr}\left[\hat{J}_{2}^{2}\right]+n \operatorname{Tr}\left[\hat{J}_{1} \hat{J}_{2} \hat{J}_{1} \hat{J}_{2}\right]\right)=-\frac{2}{2 n+1}
$$

where we have used that $\hat{J}_{1}^{2}$ is the $2 \mathrm{x} 2$ identity matrix and $\operatorname{Tr}\left[\hat{J}_{1} \hat{J}_{2} \hat{J}_{1} \hat{J}_{2}\right]=-\operatorname{Tr}\left[\hat{J}_{2}^{2}\right]=2$. Substituting:

$$
\mathcal{L}_{A^{2}}=-8 g_{x x}^{-1} \sqrt{-\operatorname{det} g} \sum_{k=0}^{\infty} \frac{(-1)^{k}}{k !} \tau^{2 k+2} \sum_{j=0}^{\infty} s_{j} j\left(g_{z z}^{-1}\left(\partial_{z} \tau\right)^{2}\right)^{j-1} A_{\nu} A^{\nu} \frac{1}{2 k+2 j-1}
$$

We should now resum the series. Let us use the identity: ${ }^{16}$

$$
\sum_{i=0}^{\infty} \frac{1}{i !} x^{i} \sum_{j=0}^{\infty} \frac{j s_{j}}{2 i+2 j-1} y^{j}=\frac{y}{2} \int_{0}^{1} \frac{e^{x a^{2}}}{\sqrt{1+y a^{2}}} d a
$$

Using $x=-\tau^{2}$ and $y=g_{z z}^{-1}\left(\partial_{z} \tau\right)^{2}$, we finally find:

$$
\mathcal{L}_{A^{2}}=-4 g_{x x}^{-1} \sqrt{-\operatorname{det} g} \tau^{2} A_{\nu} A^{\nu} \int_{0}^{1} \frac{e^{-\tau^{2} a^{2}}}{\sqrt{1+g_{z z}^{-1}\left(\partial_{z} \tau\right)^{2} a^{2}}} d a
$$

Let us now compute the $F^{2}$ terms. We want to expand the determinant of (H.1) to second order in $\hat{F}$ but to all orders in $D_{z} \hat{T} D_{z} \hat{T}$. The determinant reads:

$$
\begin{aligned}
-\operatorname{det}\left(g_{M N}+\right. & \left.\hat{F}_{M N}+D_{M} \hat{T} D_{N} \hat{T}\right)= \\
& g_{x x}^{4}\left(g_{z z}+D_{z} \hat{T} D_{z} \hat{T}\right)+\frac{1}{2} \hat{F}_{\mu \nu} \hat{F}^{\mu \nu} g_{x x}^{2}\left(g_{z z}+D_{z} \hat{T} D_{z} \hat{T}\right)+g_{x x}^{3} \hat{F}_{\mu z}^{2}
\end{aligned}
$$

and thus the $F^{2}$ contribution to the square root is:

$$
\frac{1}{4} g_{z z}^{\frac{1}{2}} \sqrt{\left(1+g_{z z}^{-1} D_{z} \hat{T} D_{z} \hat{T}\right)} \hat{F}_{\mu \nu} \hat{F}^{\mu \nu}+\frac{1}{2} g_{x x} g_{z z}^{-\frac{1}{2}} \frac{1}{\sqrt{1+g_{z z}^{-1} D_{z} \hat{T} D_{z} \hat{T}}} \hat{F}_{\mu z}^{2}
$$

Let us start by computing the term with $\hat{F}_{\mu \nu} \hat{F}^{\mu \nu}$. We have to expand before taking the symmetrized trace. Notice that now $D_{z} \hat{T}=\partial_{z} \tau \hat{J}_{1}$ up to subleading terms. To shorten notation, we define:

$$
x \equiv-\tau^{2}, \quad y=g_{z z}^{-1}\left(\partial_{z} \tau\right)^{2}
$$

The $\hat{F}_{\mu \nu} \hat{F}^{\mu \nu}$ term in the lagrangian density (H.1) then reads:

$$
\mathcal{L}_{F_{\mu \nu}^{2}}=-\frac{1}{4} g_{z z}^{\frac{1}{2}} \sum_{k=0}^{\infty} \frac{x^{k}}{k !} \sum_{j=0}^{\infty} s_{j} y^{j} \operatorname{STr}\left[\hat{J}_{1}^{2 k+2 j} \hat{F}_{\mu \nu} \hat{F}^{\mu \nu}\right]
$$

\footnotetext{
${ }^{16}$ In order to prove this, notice that $\sum_{j=0}^{\infty} j s_{j} y^{j}=\frac{y}{2 \sqrt{1+y}}$ and consider an auxiliary function $g(a)=$ $\sum_{i=0}^{\infty} \frac{1}{i !} x^{i} \sum_{j=0}^{\infty} \frac{j s_{j}}{2 i+2 j-1} y^{j} a^{2 i+2 j-1}$ such that $\partial_{a} g(a)=a^{-2} e^{x a^{2}} \frac{a^{2} y}{2 \sqrt{1+a^{2} y}}$. Since $g(0)=0$ and $g(1)$ is what we want to compute, we arrive at (H.12).
} 
It is now easy to compute the symmetriced trace:

$$
\mathrm{S} \operatorname{Tr}\left[\hat{J}_{1}^{2 k+2 j} \hat{F}_{\mu \nu} \hat{F}^{\mu \nu}\right]=\frac{1}{2 k+2 j+1}\left((k+j+1) \operatorname{Tr}\left[\hat{F}_{\mu \nu} \hat{F}^{\mu \nu}\right]+(k+j) \operatorname{Tr}\left[\hat{J}_{1} \hat{F}_{\mu \nu} \hat{J}_{1} \hat{F}^{\mu \nu}\right]\right)
$$

Now, $\operatorname{Tr}\left[\hat{F}_{\mu \nu} \hat{F}^{\mu \nu}\right]=F_{\mu \nu}^{(L)} F^{\mu \nu(L)}+F_{\mu \nu}^{(R)} F^{\mu \nu(R)}$ which, splitting in vector and axial part and using notation of section 4 gives $\operatorname{Tr}\left[\hat{F}_{\mu \nu} \hat{F}^{\mu \nu}\right]=2 V_{\mu \nu} V^{\mu \nu}+2 A_{\mu \nu} A^{\mu \nu}$. Similarly, $\operatorname{Tr}\left[\hat{J}_{1} \hat{F}_{\mu \nu} \hat{J}_{1} \hat{F}^{\mu \nu}\right]=2 F_{\mu \nu}^{(L)} F^{\mu \nu(R)}=2 V_{\mu \nu} V^{\mu \nu}-2 A_{\mu \nu} A^{\mu \nu}$ and we find $\operatorname{STr}\left[\hat{J}_{1}^{2 k+2 j} \hat{F}_{\mu \nu} \hat{F}^{\mu \nu}\right]=$ $2 V_{\mu \nu} V^{\mu \nu}+\frac{2}{2 i+2 j+1} A_{\mu \nu} A^{\mu \nu}$. Inserting this in (H.17):

$$
\begin{aligned}
\mathcal{L}_{F_{\mu \nu}^{2}} & =-\frac{1}{4} g_{z z}^{\frac{1}{2}} \sum_{k=0}^{\infty} \frac{x^{k}}{k !} \sum_{j=0}^{\infty} s_{j} y^{j}\left(2 V_{\mu \nu} V^{\mu \nu}+\frac{2}{2 i+2 j+1} A_{\mu \nu} A^{\mu \nu}\right)= \\
& =-\frac{1}{2} g_{z z}^{\frac{1}{2}}\left[e^{x} \sqrt{1+y} V_{\mu \nu} V^{\mu \nu}+\left(\int_{0}^{1} e^{a^{2} x} \sqrt{1+a^{2} y} d a\right) A_{\mu \nu} A^{\mu \nu}\right]
\end{aligned}
$$

The fact that for non-trivial tachyon the symmetric trace produces a coupling between the left and right gauge fields was already pointed out in [38]. It results in different kinetic terms for vectors and axials. We skip the details of the similar computation leading to $\hat{F}_{\mu z}^{2}$ :

$$
\mathcal{L}_{F_{\mu z}^{2}}=-g_{x x} g_{z z^{2}}^{-\frac{1}{2}}\left[e^{x} \frac{1}{\sqrt{1+y}}\left(\partial_{z} V_{\mu}\right)^{2}+\left(\int_{0}^{1} e^{a^{2} x} \frac{1}{\sqrt{1+a^{2} y}} d a\right)\left(\partial_{z} A_{\mu}\right)^{2}\right]
$$

By comparing (H.19), (H.20) to (4.5), we find that the quadratic action for the vector excitation is identical regardless the choice between Sen's and Garousi's actions. Nevertheless, the axial part changes. From (H.19), (H.20) it can be read that, introducing notation of appendix D:

$$
\mathcal{L}_{\text {axial }}=-\left[\frac{1}{2} B(z) A_{\mu \nu} A^{\mu \nu}+A(z)\left(\partial_{z} A_{\mu}\right)^{2}+M(z) A_{\mu}^{2}\right]
$$

with:

$$
\begin{aligned}
& A(z)=g_{x x} g_{z z^{-\frac{1}{2}}} \int_{0}^{1} e^{-\tau^{2} a^{2}}\left(\sqrt{1+a^{2} g_{z z}^{-1}\left(\partial_{z} \tau\right)^{2}}\right)^{-1} d a \\
& B(z)=g_{z z}^{\frac{1}{2}} \int_{0}^{1} e^{-\tau^{2} a^{2}} \sqrt{1+a^{2} g_{z z}^{-1}\left(\partial_{z} \tau\right)^{2}} d a \\
& M(z)=4 g_{x x} g_{z z}^{\frac{1}{2}} \tau^{2} \int_{0}^{1} e^{-\tau^{2} a^{2}}\left(\sqrt{1+a^{2} g_{z z}^{-1}\left(\partial_{z} \tau\right)^{2}}\right)^{-1} d a
\end{aligned}
$$

In order to proceed further, we need estimate the integrals near the IR, where $z \rightarrow z_{\Lambda}$ and the tachyon diverges, see section 3.1. We will use that in the limit where $-x \gg 1$ and $y \gg 1$ with $-\frac{y}{x} \gg 1$, it happens that:

$$
\int_{0}^{1} e^{x a^{2}} \sqrt{1+y a^{2}} d a \approx-\frac{\sqrt{y}}{2 x}, \quad \int_{0}^{1} e^{x a^{2}}\left(\sqrt{1+y a^{2}}\right)^{-1} d a \approx \frac{\log \left(\frac{y}{-x}\right)-\gamma+2 \log 2}{2 \sqrt{y}}
$$

where $\gamma$ is Euler's constant. The first equality is just found by neglecting the 1 inside the square root. For the second computation, it is not possible to directly neglect the 1 since 
the result would be divergent, but one can express the integral as $\int_{0}^{1}\left(\sqrt{1+y a^{2}}\right)^{-1} d a+$ $\int_{0}^{1}\left(e^{x a^{2}}-1\right)\left(\sqrt{1+y a^{2}}\right)^{-1} d a$, such that the first integral can be done explicitly and in the second one the 1 inside the square root can be neglected.

We are now ready to compute the leading IR behaviour of the Schrödinger potential which will determine the behaviour of the highly excited states. We will use that near the IR $\left(z_{\Lambda}-z \ll 1\right)$, we have:

$$
\begin{aligned}
g_{x x} & \approx \frac{R^{2}}{z_{\Lambda}^{2}}, \quad g_{z z} \approx \frac{R^{2}}{z_{\Lambda}^{2}} \frac{z_{\Lambda}}{5\left(z_{\Lambda}-z\right)}, \quad \tau=\sqrt{-x} \approx C\left(z_{\Lambda}-z\right)^{-\frac{3}{20}} \\
g_{z z}^{-1}\left(\partial_{z} \tau\right)^{2}=y & \approx \frac{9 z_{\Lambda} C^{2}}{80 R^{2}}\left(z_{\Lambda}-z\right)^{-\frac{13}{10}}
\end{aligned}
$$

From (H.22)-(H.24) one can readily check that $\lim _{z \rightarrow z_{\Lambda}} M(z) / B(z)=0$ and therefore the term $h(u)$ does not contribute in the IR to the Schrödinger potential (D.7). On the other hand, we can obtain the relation of the $z$-coordinate to the $u$-coordinate of the Schrödinger problem (D.5):

$$
\sqrt{\frac{B(z)}{A(z)}} \approx \frac{3 z_{\Lambda}}{20} \frac{\left(z_{\Lambda}-z\right)^{-1}}{\sqrt{-\log \left(b\left(z_{\Lambda}-z\right)\right)}}, \quad u \approx \frac{3}{10} z_{\Lambda} \sqrt{-\log \left(b\left(z_{\Lambda}-z\right)\right)} .
$$

where $b$ is a positive constant that will not be important in the following. We also compute:

$$
\Xi=(A B)^{\frac{1}{4}} \sim e^{-\frac{5 u^{2}}{6 z_{\Lambda}^{2}}}
$$

where we have not written multiplicative constants and powers of $u$ which do not affect the leading IR behaviour of the Schrödinger potential. Finally, from (D.7) we find

$$
V(u) \approx \frac{25}{9 z_{\Lambda}^{4}} u^{2}
$$

Since we have a quadratic potential in the IR, the behaviour for asymptotically highly excited axials is still Regge-like. Unlike in the main text - section 4.2.1 — the slope found using Garousi's action does not depend on the constant $k$. Comparing to the vector modes - section 4.1.1 - we see that the Regge slope for the axials is slightly larger, in particular $\Lambda_{A}^{2} / \Lambda_{V}^{2}=10 / 9$, where $\Lambda_{V, A}$ are defined as in (6.1).

Open Access. This article is distributed under the terms of the Creative Commons Attribution Noncommercial License which permits any noncommercial use, distribution, and reproduction in any medium, provided the original author(s) and source are credited.

\section{References}

[1] J.M. Maldacena, The large-N limit of superconformal field theories and supergravity, Int. J. Theor. Phys. 38 (1999) 1113 [Adv. Theor. Math. Phys. 2 (1998) 231] [hep-th/9711200] [SPIRES].

[2] S.S. Gubser, I.R. Klebanov and A.M. Polyakov, Gauge theory correlators from non-critical string theory, Phys. Lett. B 428 (1998) 105 [hep-th/9802109] [SPIRES]. 
[3] E. Witten, Anti-de Sitter space and holography, Adv. Theor. Math. Phys. 2 (1998) 253 [hep-th/9802150] [SPIRES].

[4] D. Mateos, String Theory and Quantum Chromodynamics, Class. Quant. Grav. 24 (2007) S713 [arXiv:0709.1523] [SPIRES].

[5] E. Witten, Anti-de Sitter space, thermal phase transition and confinement in gauge theories, Adv. Theor. Math. Phys. 2 (1998) 505 [hep-th/9803131] [SPIRES].

[6] J.M. Maldacena and C. Núñez, Towards the large- $N$ limit of pure $N=1$ super Yang-Mills, Phys. Rev. Lett. 86 (2001) 588 [hep-th/0008001] [SPIRES].

[7] A.H. Chamseddine and M.S. Volkov, Non-Abelian BPS monopoles in $N=4$ gauged supergravity, Phys. Rev. Lett. 79 (1997) 3343 [hep-th/9707176] [SPIRES].

[8] I.R. Klebanov and M.J. Strassler, Supergravity and a confining gauge theory: Duality cascades and $\chi S B$-resolution of naked singularities, JHEP 08 (2000) 052 [hep-th/0007191] [SPIRES].

[9] J. Polchinski and M.J. Strassler, Hard scattering and gauge/string duality, Phys. Rev. Lett. 88 (2002) 031601 [hep-th/0109174] [SPIRES].

[10] U. Gürsoy and E. Kiritsis, Exploring improved holographic theories for QCD: Part I, JHEP 02 (2008) 032 [arXiv:0707.1324] [SPIRES].

[11] U. Gürsoy, E. Kiritsis and F. Nitti, Exploring improved holographic theories for QCD: Part II, JHEP 02 (2008) 019 [arXiv: 0707.1349] [SPIRES].

[12] U. Gürsoy, E. Kiritsis, L. Mazzanti and F. Nitti, Deconfinement and Gluon Plasma Dynamics in Improved Holographic QCD, Phys. Rev. Lett. 101 (2008) 181601 [arXiv: 0804.0899] [SPIRES].

[13] U. Gürsoy, E. Kiritsis, L. Mazzanti and F. Nitti, Holography and Thermodynamics of $5 D$ Dilaton-gravity, JHEP 05 (2009) 033 [arXiv:0812.0792] [SPIRES].

[14] U. Gürsoy, E. Kiritsis, L. Mazzanti and F. Nitti, Improved Holographic Yang-Mills at Finite Temperature: Comparison with Data, Nucl. Phys. B 820 (2009) 148 [arXiv:0903.2859] [SPIRES].

[15] U. Gürsoy, E. Kiritsis, G. Michalogiorgakis and F. Nitti, Thermal Transport and Drag Force in Improved Holographic QCD, JHEP 12 (2009) 056 [arXiv:0906.1890] [SPIRES].

[16] J. Babington, J. Erdmenger, N.J. Evans, Z. Guralnik and I. Kirsch, Chiral symmetry breaking and pions in non-supersymmetric gauge / gravity duals, Phys. Rev. D 69 (2004) 066007 [hep-th/0306018] [SPIRES].

[17] N.J. Evans and J.P. Shock, Chiral dynamics from AdS space, Phys. Rev. D 70 (2004) 046002 [hep-th/0403279] [SPIRES].

[18] M. Kruczenski, D. Mateos, R.C. Myers and D.J. Winters, Towards a holographic dual of large- $N_{c}$ QCD, JHEP 05 (2004) 041 [hep-th/0311270] [SPIRES].

[19] T. Sakai and S. Sugimoto, Low energy hadron physics in holographic QCD, Prog. Theor. Phys. 113 (2005) 843 [hep-th/0412141] [SPIRES].

[20] J. Erlich, E. Katz, D.T. Son and M.A. Stephanov, QCD and a Holographic Model of Hadrons, Phys. Rev. Lett. 95 (2005) 261602 [hep-ph/0501128] [SPIRES].

[21] L. Da Rold and A. Pomarol, Chiral symmetry breaking from five dimensional spaces, Nucl. Phys. B 721 (2005) 79 [hep-ph/0501218] [SPIRES]. 
[22] A. Karch, E. Katz, D.T. Son and M.A. Stephanov, Linear Confinement and AdS/QCD, Phys. Rev. D 74 (2006) 015005 [hep-ph/0602229] [SPIRES].

[23] I. Iatrakis, E. Kiritsis and A. Paredes, An AdS/QCD model from Sen's tachyon action, Phys. Rev. D 81 (2010) 115004 [arXiv:1003.2377] [SPIRES].

[24] R. Casero, E. Kiritsis and A. Paredes, Chiral symmetry breaking as open string tachyon condensation, Nucl. Phys. B 787 (2007) 98 [hep-th/0702155] [SPIRES].

[25] A. Sen, Dirac-Born-Infeld action on the tachyon kink and vortex, Phys. Rev. D 68 (2003) 066008 [hep-th/0303057] [SPIRES].

[26] M. Kruczenski, D. Mateos, R.C. Myers and D.J. Winters, Meson spectroscopy in AdS/CFT with flavour, JHEP 07 (2003) 049 [hep-th/0304032] [SPIRES].

[27] J. Erdmenger, N. Evans, I. Kirsch and E. Threlfall, Mesons in Gauge/Gravity Duals - A Review, Eur. Phys. J. A 35 (2008) 81 [arXiv:0711.4467] [SPIRES];

[28] S. Kuperstein and J. Sonnenschein, Non-critical supergravity $(d>1)$ and holography, JHEP 07 (2004) 049 [hep-th/0403254] [SPIRES].

[29] S. Kuperstein and J. Sonnenschein, Non-critical, near extremal AdS $S_{6}$ background as a holographic laboratory of four dimensional YM theory, JHEP 11 (2004) 026 [hep-th/0411009] [SPIRES].

[30] I.R. Klebanov and J.M. Maldacena, Superconformal gauge theories and non-critical superstrings, Int. J. Mod. Phys. A 19 (2004) 5003 [hep-th/0409133] [SPIRES].

[31] F. Bigazzi, R. Casero, A.L. Cotrone, E. Kiritsis and A. Paredes, Non-critical holography and four-dimensional CFT's with fundamentals, JHEP 10 (2005) 012 [hep-th/0505140] [SPIRES].

[32] R. Casero, A. Paredes and J. Sonnenschein, Fundamental matter, meson spectroscopy and non-critical string/gauge duality, JHEP 01 (2006) 127 [hep-th/0510110] [SPIRES].

[33] V. Mazu and J. Sonnenschein, Non critical holographic models of the thermal phases of QCD, JHEP 06 (2008) 091 [arXiv: 0711.4273] [SPIRES].

[34] O. Mintakevich and J. Sonnenschein, On the spectra of scalar mesons from HQCD models, JHEP 08 (2008) 082 [arXiv:0806.0152] [SPIRES].

[35] E. Witten, Anti-de Sitter space, thermal phase transition and confinement in gauge theories, Adv. Theor. Math. Phys. 2 (1998) 505 [hep-th/9803131] [SPIRES].

[36] D. Kutasov, M. Mariño and G.W. Moore, Some exact results on tachyon condensation in string field theory, JHEP 10 (2000) 045 [hep-th/0009148] [SPIRES].

[37] T. Takayanagi, S. Terashima and T. Uesugi, Brane-antibrane action from boundary string field theory, JHEP 03 (2001) 019 [hep-th/0012210] [SPIRES].

[38] M.R. Garousi, On the effective action of D-brane-anti-D-brane system, JHEP 12 (2007) 089 [arXiv:0710.5469] [SPIRES].

[39] A. Pomarol and A. Wulzer, Baryon Physics in Holographic QCD, Nucl. Phys. B 809 (2009) 347 [arXiv:0807.0316] [SPIRES].

[40] O. Domenech, G. Panico and A. Wulzer, Massive Pions, Anomalies and Baryons in Holographic QCD, arXiv:1009.0711 [SPIRES].

[41] S.R. Coleman and E. Witten, Chiral Symmetry Breakdown in Large-N Chromodynamics, Phys. Rev. Lett. 45 (1980) 100 [SPIRES]. 
[42] O. Aharony, J. Sonnenschein and S. Yankielowicz, A holographic model of deconfinement and chiral symmetry restoration, Annals Phys. 322 (2007) 1420 [hep-th/0604161] [SPIRES].

[43] L. Da Rold and A. Pomarol, The scalar and pseudoscalar sector in a five-dimensional approach to chiral symmetry breaking, JHEP 01 (2006) 157 [hep-ph/0510268] [SPIRES].

[44] A. Cherman, T.D. Cohen and E.S. Werbos, The chiral condensate in holographic models of QCD, Phys. Rev. C 79 (2009) 045203 [arXiv:0804.1096] [SPIRES].

[45] U. Gürsoy and E. Kiritsis, Exploring improved holographic theories for QCD: Part I, JHEP 02 (2008) 032 [arXiv:0707.1324] [SPIRES].

[46] U. Gürsoy, E. Kiritsis and F. Nitti, Exploring improved holographic theories for QCD: Part II, JHEP 02 (2008) 019 [arXiv:0707.1349] [SPIRES].

[47] U. Gürsoy, E. Kiritsis, L. Mazzanti, G. Michalogiorgakis and F. Nitti, Improved Holographic $Q C D$, arXiv: 1006.5461 [SPIRES].

[48] K. Skenderis, Lecture notes on holographic renormalization, Class. Quant. Grav. 19 (2002) 5849 [hep-th/0209067] [SPIRES].

[49] F. Jugeau, Towards a consistent AdS/QCD dictionary, arXiv:0902.3864 [SPIRES].

[50] WuPpertal-BudAPEST collaboration, S. Borsányi et al., Is there still any Tc mystery in lattice QCD? Results with physical masses in the continuum limit III, JHEP 09 (2010) 073 [arXiv: 1005.3508] [SPIRES].

[51] E. Schreiber, Excited mesons and quantization of string endpoints, hep-th/0403226 [SPIRES].

[52] M. Gell-Mann, R.J. Oakes and B. Renner, Behavior of current divergences under SU(3) x SU(3), Phys. Rev. 175 (1968) 2195 [SPIRES].

[53] Particle Data Group collaboration, C. Amsler et al., Review of particle physics, Phys. Lett. B 667 (2008) 1 [SPIRES].

[54] C. Hoyos-Badajoz, K. Landsteiner and S. Montero, Holographic Meson Melting, JHEP 04 (2007) 031 [hep-th/0612169] [SPIRES].

[55] D. Mateos, R.C. Myers and R.M. Thomson, Holographic phase transitions with fundamental matter, Phys. Rev. Lett. 97 (2006) 091601 [hep-th/0605046] [SPIRES].

[56] D. Mateos, R.C. Myers and R.M. Thomson, Thermodynamics of the brane, JHEP 05 (2007) 067 [hep-th/0701132] [SPIRES].

[57] H.R. Grigoryan, P.M. Hohler and M.A. Stephanov, Towards the Gravity Dual of Quarkonium in the Strongly Coupled QCD Plasma, Phys. Rev. D 82 (2010) 026005 [arXiv:1003.1138] [SPIRES].

[58] M. Fujita, T. Kikuchi, K. Fukushima, T. Misumi and M. Murata, Melting Spectral Functions of the Scalar and Vector Mesons in a Holographic QCD Model, Phys. Rev. D 81 (2010) 065024 [arXiv:0911.2298] [SPIRES].

[59] A. Paredes, K. Peeters and M. Zamaklar, Mesons versus quasi-normal modes: undercooling and overheating, JHEP 05 (2008) 027 [arXiv: 0803.0759] [SPIRES].

[60] D.T. Son and A.O. Starinets, Minkowski-space correlators in AdS/CFT correspondence: Recipe and applications, JHEP 09 (2002) 042 [hep-th/0205051] [SPIRES].

[61] T. Gherghetta, J.I. Kapusta and T.M. Kelley, Chiral symmetry breaking in the soft-wall AdS/QCD model, Phys. Rev. D 79 (2009) 076003 [arXiv:0902.1998] [SPIRES]. 
[62] Y.-Q. Sui, Y.-L. Wu, Z.-F. Xie and Y.-B. Yang, Prediction for the Mass Spectra of Resonance Mesons in the Soft-Wall AdS/QCD with a Modified 5D Metric, Phys. Rev. D 81 (2010) 014024 [arXiv:0909.3887] [SPIRES].

[63] J. Sonnenschein, What does the string/gauge correspondence teach us about Wilson loops?, hep-th/0003032 [SPIRES].

[64] J.P. Shock and F. Wu, Three flavour QCD from the holographic principle, JHEP 08 (2006) 023 [hep-ph/0603142] [SPIRES].

[65] A.L. Cotrone, A. Dymarsky and S. Kuperstein, On Vector Meson Masses in a Holographic $S Q C D$, arXiv: 1010.1017 [SPIRES].

[66] C. Kennedy and A. Wilkins, Ramond-Ramond couplings on brane-antibrane systems, Phys. Lett. B 464 (1999) 206 [hep-th/9905195] [SPIRES].

[67] P. Kraus and F. Larsen, Boundary string field theory of the DD-bar system, Phys. Rev. D 63 (2001) 106004 [hep-th/0012198] [SPIRES].

[68] M.A. Shifman, A.I. Vainshtein and V.I. Zakharov, QCD and Resonance Physics. Sum Rules, Nucl. Phys. B 147 (1979) 385 [SPIRES].

[69] V.A. Novikov, M.A. Shifman, A.I. Vainshtein and V.I. Zakharov, Are All Hadrons Alike? Technical Appendices, Nucl. Phys. B 191 (1981) 301 [SPIRES].

[70] J. Gasser and H. Leutwyler, Chiral Perturbation Theory to One Loop, Ann. Phys. 158 (1984) 142 [SPIRES].

[71] G. Ecker, J. Gasser, A. Pich and E. de Rafael, The Role of Resonances in Chiral Perturbation Theory, Nucl. Phys. B 321 (1989) 311 [SPIRES].

[72] A. Pich, Colourless mesons in a polychromatic world, hep-ph/0205030 [SPIRES].

[73] N. Brambilla, A. Pineda, J. Soto and A. Vairo, Effective field theories for heavy quarkonium, Rev. Mod. Phys. 77 (2005) 1423 [hep-ph/0410047] [SPIRES].

[74] J. Soto, Overview of Non-Relativistic QCD, Eur. Phys. J. A 31 (2007) 705 [nucl-th/0611055] [SPIRES].

[75] S. Sugimoto and K. Takahashi, QED and string theory, JHEP 04 (2004) 051 [hep-th/0403247] [SPIRES].

[76] A.L. Cotrone, On the YM and QCD spectra from five dimensional strings, Int. J. Mod. Phys. A 24 (2009) 4117 [arXiv:0707.1483] [SPIRES].

[77] O. Aharony and D. Kutasov, Holographic Duals of Long Open Strings, Phys. Rev. D 78 (2008) 026005 [arXiv:0803.3547] [SPIRES].

[78] E. Kiritsis, Dissecting the string theory dual of QCD, Fortsch. Phys. 57 (2009) 396 [arXiv:0901.1772] [SPIRES].

[79] M.R. Garousi, Tachyon couplings on non-BPS D-branes and Dirac-Born-Infeld action, Nucl. Phys. B 584 (2000) 284 [hep-th/0003122] [SPIRES].

[80] K. Bitaghsir-Fadafan and M.R. Garousi, Non-abelian expansion of S-matrix elements and non-abelian tachyon DBI action, Nucl. Phys. B 760 (2007) 197 [hep-th/0607249] [SPIRES].

[81] M.R. Garousi and E. Hatefi, On Wess-Zumino terms of Brane-Antibrane systems, Nucl. Phys. B 800 (2008) 502 [arXiv:0710.5875] [SPIRES].

[82] M.R. Garousi and E. Hatefi, More on WZ action of non-BPS branes, JHEP 03 (2009) 008 [arXiv:0812.4216] [SPIRES]. 
[83] N. Gutierrez and Y. Lozano, Confinement and Non-perturbative Tachyons in Brane-Antibrane Systems, Phys. Rev. D 79 (2009) 046010 [arXiv:0809.1005] [SPIRES].

[84] M.R. Garousi, D-brane anti-D-brane effective action and brane interaction in open string channel, JHEP 01 (2005) 029 [hep-th/0411222] [SPIRES].

[85] O. Bergman, S. Seki and J. Sonnenschein, Quark mass and condensate in HQCD, JHEP 12 (2007) 037 [arXiv:0708.2839] [SPIRES].

[86] A. Dhar and P. Nag, Sakai-Sugimoto model, Tachyon Condensation and Chiral symmetry Breaking, JHEP 01 (2008) 055 [arXiv: 0708.3233] [SPIRES].

[87] A. Dhar and P. Nag, Tachyon condensation and quark mass in modified Sakai-Sugimoto model, Phys. Rev. D 78 (2008) 066021 [arXiv:0804.4807] [SPIRES].

[88] V. Niarchos, Hairpin-Branes and Tachyon-Paperclips in Holographic Backgrounds, Nucl. Phys. B 841 (2010) 268 [arXiv:1005.1650] [SPIRES].

[89] D. Erkal, D. Kutasov and O. Lunin, Brane-Antibrane Dynamics From the Tachyon DBI Action, arXiv: 0901.4368 [SPIRES].

[90] M. Golterman and S. Peris, Large- $N_{c} Q C D$ meetsRegge theory: The example of spin-one two-point functions, JHEP 01 (2001) 028 [hep-ph/0101098] [SPIRES].

[91] S.R. Beane, Constraining quark hadron duality at large- $N_{c}$, Phys. Rev. D 64 (2001) 116010 [hep-ph/0106022] [SPIRES].

[92] M. Golterman and S. Peris, On the use of the operator product expansion to constrain the hadron spectrum, Phys. Rev. D 67 (2003) 096001 [hep-ph/0207060] [SPIRES].

[93] M. Shifman, Highly excited hadrons in QCD and beyond, hep-ph/0507246 [SPIRES].

[94] M. Shifman and A. Vainshtein, Highly Excited Mesons, LinearRegge Trajectories and the Pattern of the Chiral Symmetry Realization, Phys. Rev. D 77 (2008) 034002 [arXiv:0710.0863] [SPIRES].

[95] A.A. Andrianov and D. Espriu, Parity doubling from Weinberg sum rules, Phys. Lett. B 671 (2009) 275 [arXiv:0803.4104] [SPIRES].

[96] O. Catà, M. Golterman and S. Peris, The operator product expansion does not imply parity doubling of hadrons, Phys. Rev. D 74 (2006) 016001 [hep-ph/0602194] [SPIRES].

[97] J. Mondejar and A. Pineda, Constraints onRegge models from perturbation theory, JHEP 10 (2007) 061 [arXiv:0704.1417] [SPIRES].

[98] J. Mondejar and A. Pineda, $1 / N_{c}$ and $1 / n$ preasymptotic corrections to Current-Current correlators, JHEP 06 (2008) 039 [arXiv:0803.3625] [SPIRES].

[99] O. Catà, Towards understandingRegge trajectories in holographic QCD, Phys. Rev. D 75 (2007) 106004 [hep-ph/0605251] [SPIRES].

[100] H.R. Grigoryan and A.V. Radyushkin, Form Factors and Wave Functions of Vector Mesons in Holographic QCD, Phys. Lett. B 650 (2007) 421 [hep-ph/0703069] [SPIRES].

[101] S.J. Brodsky and G.F. de Teramond, Light-Front Dynamics and AdS/QCD Correspondence: The Pion Form Factor in the Space- and Time-Like Regions, Phys. Rev. D 77 (2008) 056007 [arXiv: 0707.3859] [SPIRES].

[102] H.J. Kwee and R.F. Lebed, Pion Form Factor in Improved Holographic QCD Backgrounds, Phys. Rev. D 77 (2008) 115007 [arXiv:0712.1811] [SPIRES]. 
[103] Z. Abidin and C.E. Carlson, Gravitational Form Factors of Vector Mesons in an AdS/QCD Model, Phys. Rev. D 77 (2008) 095007 [arXiv:0801.3839] [SPIRES].

[104] H.R. Grigoryan and A.V. Radyushkin, Anomalous Form Factor of the Neutral Pion in Extended AdS/QCD Model with Chern-Simons Term, Phys. Rev. D 77 (2008) 115024 [arXiv: 0803.1143] [SPIRES].

[105] Z. Abidin and C.E. Carlson, Gravitational Form Factors in the Axial Sector from an AdS/QCD Model, Phys. Rev. D 77 (2008) 115021 [arXiv:0804.0214] [SPIRES].

[106] S.J. Brodsky and G.F. de Teramond, Light-Front Dynamics and AdS/QCD Correspondence: Gravitational Form Factors of Composite Hadrons, Phys. Rev. D 78 (2008) 025032 [arXiv: 0804.0452] [SPIRES].

[107] S.S. Agaev and M.A. Gomshi Nobary, Pion distribution amplitude from holographic QCD and the electromagnetic form factor $F_{\pi}\left(Q^{2}\right)$, Phys. Rev. D 77 (2008) 074014 [arXiv:0805.0993] [SPIRES].

[108] A. Cherman, T.D. Cohen and M. Nielsen, Tests of Universality of Baryon Form Factors in Holographic QCD, Nucl. Phys. Proc. Suppl. 199 (2010) 103 [arXiv:0909.5359] [SPIRES].

[109] C. Núñez, A. Paredes and A.V. Ramallo, Unquenched flavor in the gauge/gravity correspondence, Adv. High Energy Phys. 2010 (2010) 196714 [arXiv:1002.1088] [SPIRES].

[110] M. Piai, Lectures on walking technicolor, holography and gauge/gravity dualities, Adv. High Energy Phys. 2010 (4302) [arXiv: 1004.0176] [SPIRES].

[111] S.A. Hartnoll, Lectures on holographic methods for condensed matter physics, Class. Quant. Grav. 26 (2009) 224002 [arXiv:0903.3246] [SPIRES].

[112] A. Karch, A. O'Bannon and K. Skenderis, Holographic renormalization of probe D-branes in AdS/CFT, JHEP 04 (2006) 015 [hep-th/0512125] [SPIRES]. 